\title{
Shifted selectivity in protonation enables the mild deuteration of arenes through catalytic amounts of Bronsted acids in deuterated methanol
}

Oliver Fischer, Anja Hubert, Markus R. Heinrich*

Department of Chemistry and Pharmacy, Pharmaceutical Chemistry, Friedrich-Alexander-Universität Erlangen-

Nürnberg, Nikolaus-Fiebiger-Str. 10, 91058 Erlangen, Germany.

Supporting Information

Table of Contents

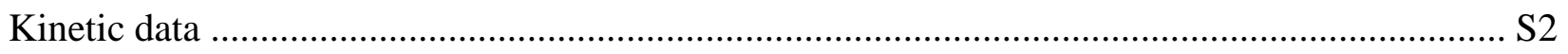

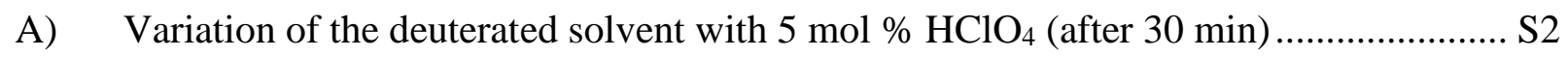

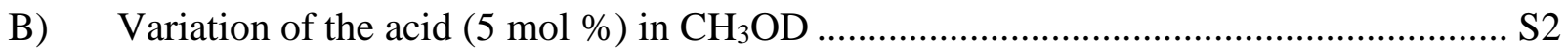

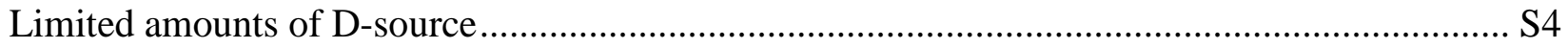

Dependence of ${ }^{1} \mathrm{H}$ NMR shift and proton exchangeability ............................................... S5

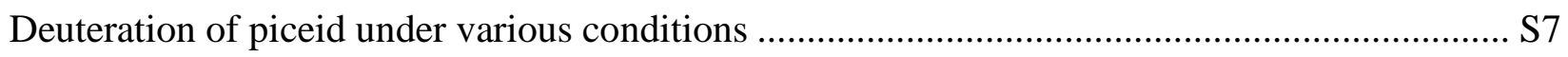

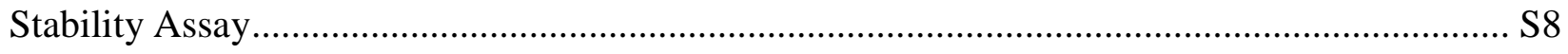

${ }^{1} \mathrm{H}$ NMR spectra of the labeled and unlabeled compounds ................................................. S9

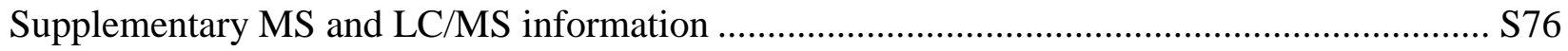




\section{KINETIC DATA}

\section{A) Variation of the deuterated solvent with 5 mol \% $\mathrm{HClO}_{4}($ after $30 \mathrm{~min})$}

Experimental procedure: 1,3,5-Trimethoxybenzene (1, $0.1 \mathrm{mmol}, 16.8 \mathrm{mg})$ was dissolved in the appropriate deuterated solvent $(1 \mathrm{~mL})$. If not stated differently, a $70 \%$ solution of $\mathrm{HClO}_{4}$ was added $(0.005 \mathrm{mmol}, 5 \mathrm{~mol} \%, 0.42 \mu \mathrm{L})$. The reaction was kept at rt under air. After 30 min, the reaction was quenched by the addition of sat. $\mathrm{NaHCO}_{3}$ (aq.) and the isotope ratios were measured via LC/MS (Single ion recording for the according masses).

Table S1: Isotope distribution of 1 after 30 min:

\begin{tabular}{|c|c|c|c|c|}
\hline & $\begin{array}{c}{\left[\mathrm{M}+3+\mathrm{H}^{+}\right]^{+}\left(\mathrm{d}_{3}\right)} \\
172 \mathrm{Da} \\
\end{array}$ & $\begin{array}{c}{\left[\mathrm{M}+2+\mathrm{H}^{+}\right]^{+}\left(\mathrm{d}_{2}\right)} \\
171 \mathrm{Da} \\
\end{array}$ & $\begin{array}{c}{\left[\mathrm{M}+\mathbf{1}+\mathrm{H}^{+}\right]^{+}\left(\mathrm{d}_{1}\right)} \\
\mathbf{1 7 0} \mathrm{Da} \\
\end{array}$ & $\begin{array}{c}{\left[\mathrm{M}+\mathrm{H}^{+}\right]^{+}\left(\mathrm{d}_{0}\right)} \\
169 \mathrm{Da} \\
\end{array}$ \\
\hline${\mathrm{MeOD}-\mathrm{d}_{4}}_{4}$ & $81 \%$ & $17 \%$ & $2 \%$ & $0 \%$ \\
\hline MeOD-d ${ }_{1}$ & $81 \%$ & $17 \%$ & $2 \%$ & $0 \%$ \\
\hline AcOD & $80 \%$ & $17 \%$ & $2 \%$ & $1 \%$ \\
\hline DCOOD & $73 \%$ & $24 \%$ & $3 \%$ & $0 \%$ \\
\hline $\mathrm{D}_{3} \mathrm{PO}_{4}$ & $68 \%$ & $23 \%$ & $7 \%$ & $2 \%$ \\
\hline $\mathrm{D}_{3} \mathrm{PO}_{4}$ (no acid added) & $63 \%$ & $25 \%$ & $9 \%$ & $3 \%$ \\
\hline EtOD-d ${ }_{1}$ & $16 \%$ & $38 \%$ & $35 \%$ & $11 \%$ \\
\hline Acetone- $\mathrm{d}_{6}$ & $5 \%$ & $22 \%$ & $42 \%$ & $31 \%$ \\
\hline $\mathrm{D}_{2} \mathrm{O}: \mathrm{MeOD} 1: 4$ & $2 \%$ & $11 \%$ & $36 \%$ & $52 \%$ \\
\hline $\mathrm{D}_{2} \mathrm{O}: \mathrm{DMSO}^{-\mathrm{d}_{6}} 4: 1$ & $1 \%$ & $5 \%$ & $26 \%$ & $68 \%$ \\
\hline AcOD (no acid added) & $0 \%$ & $2 \%$ & $15 \%$ & $82 \%$ \\
\hline $\mathrm{CDCl}_{3}$ & $0 \%$ & $1 \%$ & $12 \%$ & $86 \%$ \\
\hline $\mathrm{CD}_{2} \mathrm{Cl}_{2}$ & $0 \%$ & $1 \%$ & $11 \%$ & $88 \%$ \\
\hline $\mathrm{C}_{6} \mathrm{D}_{6}$ & $0 \%$ & $1 \%$ & $9 \%$ & $90 \%$ \\
\hline
\end{tabular}

\section{B) Variation of the acid $(5 \mathrm{~mol} \%)$ in $\mathrm{CH}_{3} \mathrm{OD}$}

Experimental procedure: 1,3,5-Trimethoxybenzene (1, $0.1 \mathrm{mmol}, 16.8 \mathrm{mg}$ ) was dissolved in $\mathrm{H}_{3} \mathrm{COD}(1 \mathrm{~mL})$. The appropriate acid was added $(0.005 \mathrm{mmol}, 5 \mathrm{~mol} \%)$. The reaction was kept at $\mathrm{rt}$ under air. After $30 \mathrm{~min}, 1 \mathrm{~h}, 6 \mathrm{~h}$, and $30 \mathrm{~h}$, the reaction was quenched by the addition of sat. $\mathrm{NaHCO}_{3}$ (aq.) and the isotope ratios were measured via LC/MS (Single ion recording for the according masses). 


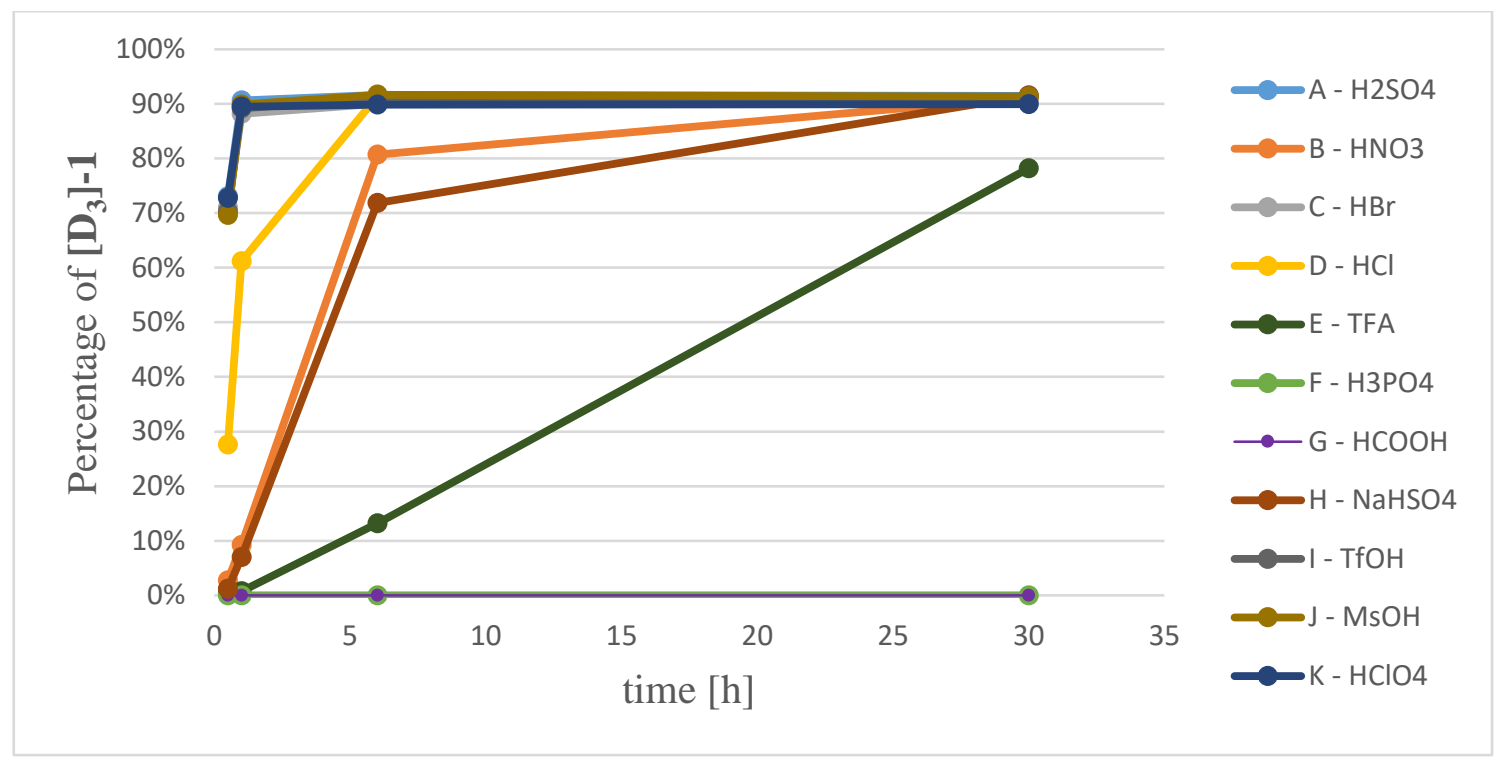

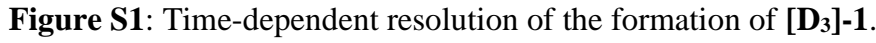

Table S2: Time-dependent formation of $\left[\mathbf{D}_{3}\right]-\mathbf{1}\left(\left[\mathrm{M}+3+\mathrm{H}^{+}\right]^{+}\left(\mathrm{d}_{3}, 172 \mathrm{Da}\right)\right)$ :

\begin{tabular}{|c|c|c|c|c|}
\hline & $30 \mathrm{~min}$ & $1 \mathrm{~h}$ & $6 \mathrm{~h}$ & $30 \mathrm{~h}$ \\
\hline $\mathrm{HClO}_{4}$ & $73 \%$ & $90 \%$ & $90 \%$ & $90 \%$ \\
\hline $\mathrm{H}_{2} \mathrm{SO}_{4}$ & $73 \%$ & $91 \%$ & $92 \%$ & $91 \%$ \\
\hline $\mathrm{HBr}$ & $71 \%$ & $88 \%$ & $90 \%$ & $91 \%$ \\
\hline $\mathrm{MsOH}$ & $70 \%$ & $90 \%$ & $92 \%$ & $91 \%$ \\
\hline TfOH & $70 \%$ & $89 \%$ & $91 \%$ & $91 \%$ \\
\hline HCl & $28 \%$ & $61 \%$ & $91 \%$ & $91 \%$ \\
\hline $\mathrm{HNO}_{3}$ & $3 \%$ & $9 \%$ & $81 \%$ & $91 \%$ \\
\hline $\mathrm{NaHSO}_{4}$ & $1 \%$ & $7 \%$ & $72 \%$ & $92 \%$ \\
\hline TFA & $0 \%$ & $1 \%$ & $13 \%$ & $78 \%$ \\
\hline $\mathrm{H}_{3} \mathrm{PO}_{4}$ & $0 \%$ & $0 \%$ & $0 \%$ & $0 \%$ \\
\hline НСООН & $0 \%$ & $0 \%$ & $0 \%$ & $0 \%$ \\
\hline
\end{tabular}




\section{LIMITED AMOUNTS OF D-SOURCE}

Experimental procedure: 1,3,5-Trimethoxybenzene (1, $0.025 \mathrm{mmol}, 4.2 \mathrm{mg})$ was dissolved in the according solvent $(0.25$ $\mathrm{mL}, 0.1 \mathrm{M})$. A $70 \%$ solution of $\mathrm{HClO}_{4}$ was added $(0.005 \mathrm{mmol}, 5 \mathrm{~mol} \%, 0.11 \mu \mathrm{L})$. The reaction was kept at $\mathrm{rt}$ under air for $10 \mathrm{~h}$ to establish an equilibrium. Then, the isotope ratios were measured via LC/MS (Single ion recording for the according masses).

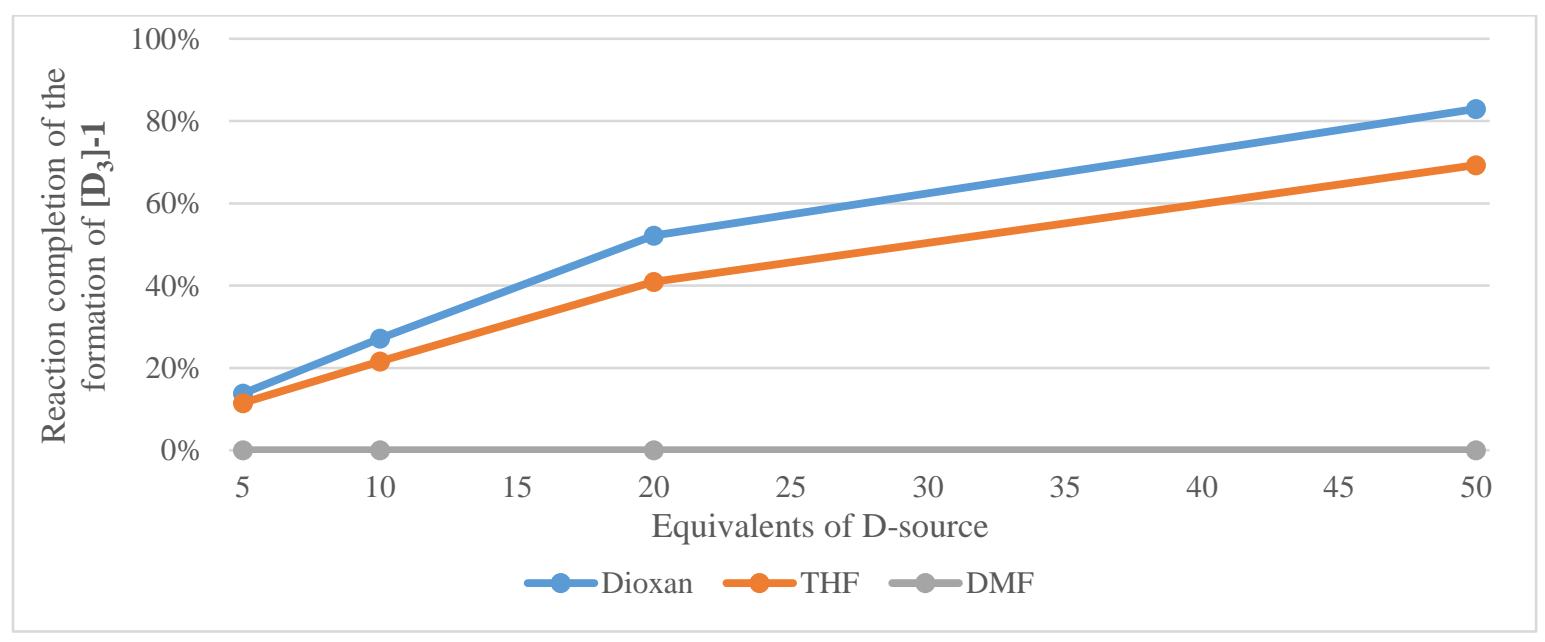

Figure S2: D-source dependent reaction completion rate of the formation of [ $\left.\mathbf{D}_{\mathbf{3}}\right]-\mathbf{1}$ in various solvents $(0.1 \mathrm{M})$ compared to the benchmark reaction in pure $\mathrm{CH}_{3} \mathrm{OD}(0.1 \mathrm{M})$.

Table S3: D-source dependent reaction completion rate of the formation of [ $\left.\mathbf{D}_{\mathbf{3}}\right] \mathbf{- 1}$ in various solvents $(0.1 \mathrm{M})$ compared to the benchmark reaction in pure $\mathrm{CH}_{3} \mathrm{OD}(0.1 \mathrm{M})$.

\begin{tabular}{|c|c|c|c|c|c|c|}
\hline & Eq. Of D-s ource & d3 & d2 & d1 & do & Reaction completion \\
\hline MeOD-d1 & 240 & $81 \%$ & $17 \%$ & $2 \%$ & $0 \%$ & $100 \%$ \\
\hline \multirow[t]{4}{*}{ Dioxan } & 5 & $11 \%$ & $32 \%$ & $39 \%$ & $17 \%$ & $14 \%$ \\
\hline & 10 & $22 \%$ & $41 \%$ & $30 \%$ & $7 \%$ & $27 \%$ \\
\hline & 20 & $42 \%$ & $42 \%$ & $14 \%$ & $2 \%$ & $52 \%$ \\
\hline & 50 & $67 \%$ & $28 \%$ & $4 \%$ & $0 \%$ & $83 \%$ \\
\hline \multirow[t]{4}{*}{ THF } & 5 & $9 \%$ & $27 \%$ & $41 \%$ & $23 \%$ & $11 \%$ \\
\hline & 10 & $17 \%$ & $40 \%$ & $32 \%$ & $11 \%$ & $22 \%$ \\
\hline & 20 & $33 \%$ & $43 \%$ & $20 \%$ & $4 \%$ & $41 \%$ \\
\hline & 50 & $56 \%$ & $36 \%$ & $8 \%$ & $1 \%$ & $69 \%$ \\
\hline \multirow[t]{4}{*}{ DMF } & 5 & $0 \%$ & $1 \%$ & $13 \%$ & $86 \%$ & $0 \%$ \\
\hline & 10 & $0 \%$ & $1 \%$ & $13 \%$ & $86 \%$ & $0 \%$ \\
\hline & 20 & $0 \%$ & $1 \%$ & $13 \%$ & $86 \%$ & $0 \%$ \\
\hline & 50 & $0 \%$ & $1 \%$ & $13 \%$ & $86 \%$ & $0 \%$ \\
\hline
\end{tabular}




\section{$\begin{array}{lllllll}\text { DEPENDENCE } & \text { OF } & { }^{1} \mathrm{H} & \text { NMR } & \text { SHIFT } & \text { AND } & \text { PROTON }\end{array}$}

\section{EXCHANGEABILITY}

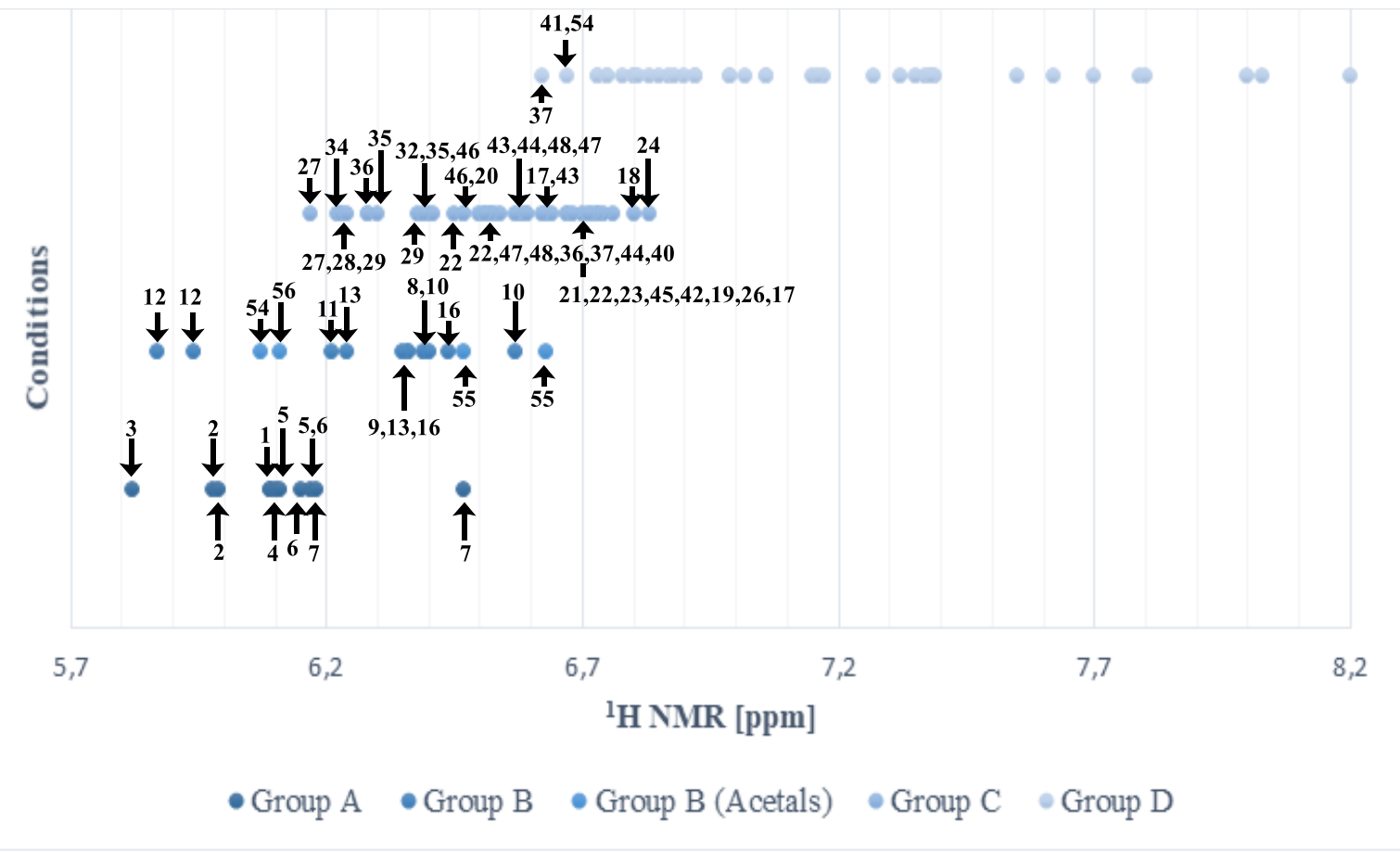

Figure S3: ${ }^{1} \mathrm{H}-\mathrm{NMR}$ shifts of aromatic positions accessible for deuteration in $\mathrm{CD}_{3} \mathrm{OD}$ with compound marker.

Table S4: ${ }^{1} \mathrm{H}-\mathrm{NMR}$ shifts of aromatic positions accessible for deuteration in $\mathrm{CD}_{3} \mathrm{OD}$. Exchangeable protons are bold:

\begin{tabular}{|c|c|c|c|c|c|}
\hline Identifier & Substrate name & \multicolumn{4}{|c|}{$\begin{array}{l}{ }^{1} \mathrm{H} \text { NMR-shifts [ppm] of aromatic } \\
\text { protons }\end{array}$} \\
\hline \multicolumn{6}{|l|}{ Group A } \\
\hline 1 & 1,3,5-Trimethoxybenzene & 6,09 & & & \\
\hline 2 & 3,5-Dimethoxyphenol & 5,98 & 5,99 & & \\
\hline 3 & 2',4',6'-Trihydroxypropiophenone & 5,82 & & & \\
\hline 4 & 1-(Allyloxy)-3,5-dimethoxybenzene & 6,09 & & & \\
\hline 5 & 1-(Benzyloxy)-3,5-dimethoxybenzene & 7,38 & 6,17 & 6,1 & \\
\hline 6 & $\begin{array}{l}\text { 2-(3,5-Dimethoxyphenoxy)-1- } \\
\text { phenylethan-1-one }\end{array}$ & 8 & 7,55 & 6,15 & 6,11 \\
\hline 7 & Resveratrol & 7,37 & 6,8 & 6,47 & 6,18 \\
\hline \multicolumn{6}{|l|}{ Group B } \\
\hline 8 & 3-Methoxyphenol & 7,06 & 6,39 & & \\
\hline 9 & 2,4,6-Trimethoxybenzaldehyde & 6,36 & & & \\
\hline 10 & 1,2,4-Trimethoxybenzene & 6,87 & 6,57 & 6,4 & \\
\hline
\end{tabular}




\begin{tabular}{|c|c|c|c|c|c|}
\hline 11 & 1-Bromo-2,4,6-trimethoxybenzene & 6,21 & & & \\
\hline 12 & 3,5-Dimethoxyaniline & 5,94 & 5,87 & & \\
\hline 13 & (p-Nitrophenyl)-dimethoxybenzene & 8,2 & 7,06 & 6,36 & 6,24 \\
\hline 16 & $\delta$-Tocopherol & 6,44 & 6,35 & & \\
\hline 54 & Neohesperidin-dihydrochalcone & 6,83 & 6,73 & 6,67 & 6,07 \\
\hline 55 & Piceid & 7,39 & 6,78 & 6,63 & 6,47 \\
\hline 56 & Hesperidin & 6,9 & 6,11 & & \\
\hline \multicolumn{6}{|l|}{ Group C } \\
\hline 17 & 2,6-Dimethoxyphenol & 6,76 & 6,63 & & \\
\hline 18 & 2-Methoxyphenol & 6,92 & 6,8 & 6,8 & \\
\hline 19 & 4-Hydroxyanisole & 6,73 & 6,73 & & \\
\hline 20 & 1,3-Dimethoxybenzene & 7,17 & 6,5 & 6,47 & \\
\hline 21 & 1,2,3-Trimethoxybenzene & 7,02 & 6,67 & & \\
\hline 22 & 1-Bromo-3,5-dimethoxybenzene & 6,67 & 6,45 & & \\
\hline 23 & 2,6-dimethoxybenzoic acid & 7,35 & 6,7 & & \\
\hline 24 & 5-Bromo-1,2,3-trimethoxybenzene & 6,83 & & & \\
\hline 26 & 2-Methoxy-4-methylphenol & 6,74 & 6,68 & & \\
\hline 27 & 3-Aminophenol & 6,91 & 6,23 & 6,17 & \\
\hline 28 & 3-(Dimethylamino)phenol & 7,01 & 6,23 & & \\
\hline 29 & 2,5-Dimethoxyaniline & 6,74 & 6,39 & 6,24 & \\
\hline 31 & 2,2',4,4'-Tetrahydroxybenzophenone & 7,38 & 6,38 & & \\
\hline 34 & IC261 & 7,79 & 7,16 & 6,99 & 6,88 \\
\hline 35 & Genistein & 7,5 & 6,9 & 6,4 & 6,3 \\
\hline 36 & Quercetin & 7,8 & 7,7 & 6,99 & 6,51 \\
\hline 37 & Hematoxylin & 6,75 & 6,62 & 6,52 & \\
\hline 40 & Chrysin & 8,03 & 7,62 & 6,8 & 6,54 \\
\hline 42 & 4-(tert-butyl)phenol & 7,22 & 6,71 & & \\
\hline 43 & $m$-Cresol & 7,04 & 6,64 & 6,62 & $\mathbf{6 , 5 7}$ \\
\hline 44 & 3-Fluorphenol & 7,15 & 6,59 & 6,53 & \\
\hline 45 & $o$-Cresol & 7,05 & 6,99 & 6,73 & 6,72 \\
\hline 46 & 3,4-Dimethylphenol & 6,47 & 6,41 & & \\
\hline 47 & 3,5-Dimethylphenol & 6,91 & 6,59 & 6,51 & \\
\hline 48 & Estradiol & 7,09 & 6,58 & 6,52 & \\
\hline \multicolumn{6}{|l|}{ Group D } \\
\hline & 1,4-Dimethoxybenzene & 6,85 & & & \\
\hline & Anisole & 7,27 & 6,92 & & \\
\hline & Paracetamol & 7,32 & 6,75 & & \\
\hline & 3,4-Dihydroxybenzoesäure & 6,81 & & & \\
\hline 41 & $p$-Cresol & 6,88 & 6,68 & & \\
\hline
\end{tabular}




\section{DEUTERATION OF PICEID UNDER VARIOUS CONDITIONS}
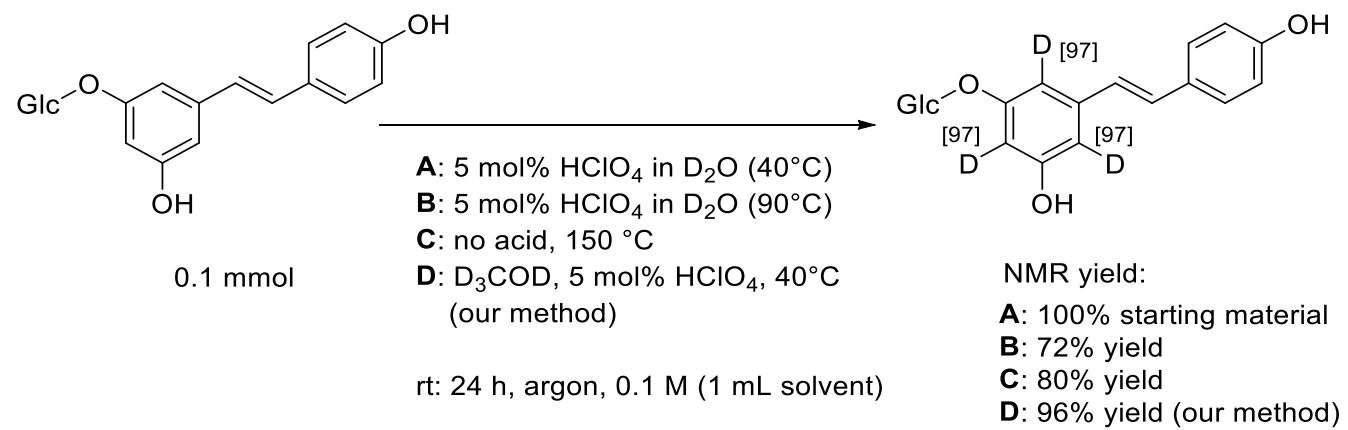

Scheme S1: Deuteration of piceid (55) under various conditions.

The deuterium incorporation and yield were determined by ${ }^{1} \mathrm{H}$ NMR analysis of the mixture in presence of ethylene carbonate as an internal standard $\left(1 \mathrm{M}\right.$ in $\left.\mathrm{d}_{4}-\mathrm{MeOD}, 50 \mu \mathrm{L}, 50 \mu \mathrm{mol}\right)$.

A: Piceid (55, $0.1 \mathrm{mmol}, 39.0 \mathrm{mg})$ was dissolved in $\mathrm{D}_{2} \mathrm{O}(1 \mathrm{~mL})$. A $70 \%$ solution of $\mathrm{HClO}_{4}(0.005 \mathrm{mmol}, 5 \mathrm{~mol} \%, 0.42 \mu \mathrm{L})$ was added and the mixture was kept at $40{ }^{\circ} \mathrm{C}$ under argon for $1 \mathrm{~d}(0 \%$ deuterium incorporation).

B: Piceid $(\mathbf{5 5}, 0.1 \mathrm{mmol}, 39.0 \mathrm{mg})$ was dissolved in $\mathrm{D}_{2} \mathrm{O}(1 \mathrm{~mL})$. A $70 \%$ solution of $\mathrm{HClO}_{4}(0.005 \mathrm{mmol}, 5 \mathrm{~mol} \%, 0.42 \mu \mathrm{L})$ was added and the mixture was kept at $90{ }^{\circ} \mathrm{C}$ under argon for $1 \mathrm{~d}(72 \mu \mathrm{mol}, 72 \%$ yield, $97 \%$ deuterium incorporation $)$.

C: Piceid $(\mathbf{5 5}, 0.1 \mathrm{mmol}, 39.0 \mathrm{mg})$ was dissolved in $\mathrm{D}_{2} \mathrm{O}(1 \mathrm{~mL})$ and the mixture was kept at $150{ }^{\circ} \mathrm{C}$ under argon for $1 \mathrm{~d}(80$ $\mu \mathrm{mol}, 80 \%$ yield, $97 \%$ deuterium incorporation).

D: Piceid $(\mathbf{5 5}, 0.1 \mathrm{mmol}, 39.0 \mathrm{mg})$ was dissolved in MeOD-d $(1 \mathrm{~mL})$. A 70\% solution of $\mathrm{HClO}_{4}(0.005 \mathrm{mmol}, 5 \mathrm{~mol} \%, 0.42$ $\mu \mathrm{L}$ ) was added and the mixture was kept at $40{ }^{\circ} \mathrm{C}$ under argon for $1 \mathrm{~d}(96 \mu \mathrm{mol}, 96 \%$ yield, $97 \%$ deuterium incorporation). 


\section{STABILITY ASSAY}

Experimental procedure: The according substrate $(0.01 \mathrm{mmol})$ was dissolved in a solution of $50: 50 \mathrm{CH} \mathrm{CN}_{3}: \mathrm{H}_{2} \mathrm{O}+0.1 \%$ formic acid $(1.0 \mathrm{~mL}, 10 \mathrm{mM})$. Over the course of the assay, the samples were kept in a closed $\mathrm{HPLC}$ vial at $10^{\circ} \mathrm{C}$. For the assay, the isotopic purity of fully deuterated compound was determined by measuring the ratio of isotopes via LC/MS and single ion recording (SIR) of the according masses. The relative change in isotopic purity of the compound compared to the starting isotopic purity was monitored via regular LC/MS runs over 7 days.

\section{$\mathrm{H}_{2} \mathrm{O}: \mathrm{CH}_{3} \mathrm{CN} 50: 50+0.1 \%$ formic acid $(1 \mathrm{~mL}, 10 \mathrm{mM})$ at $10{ }^{\circ} \mathrm{C}$}

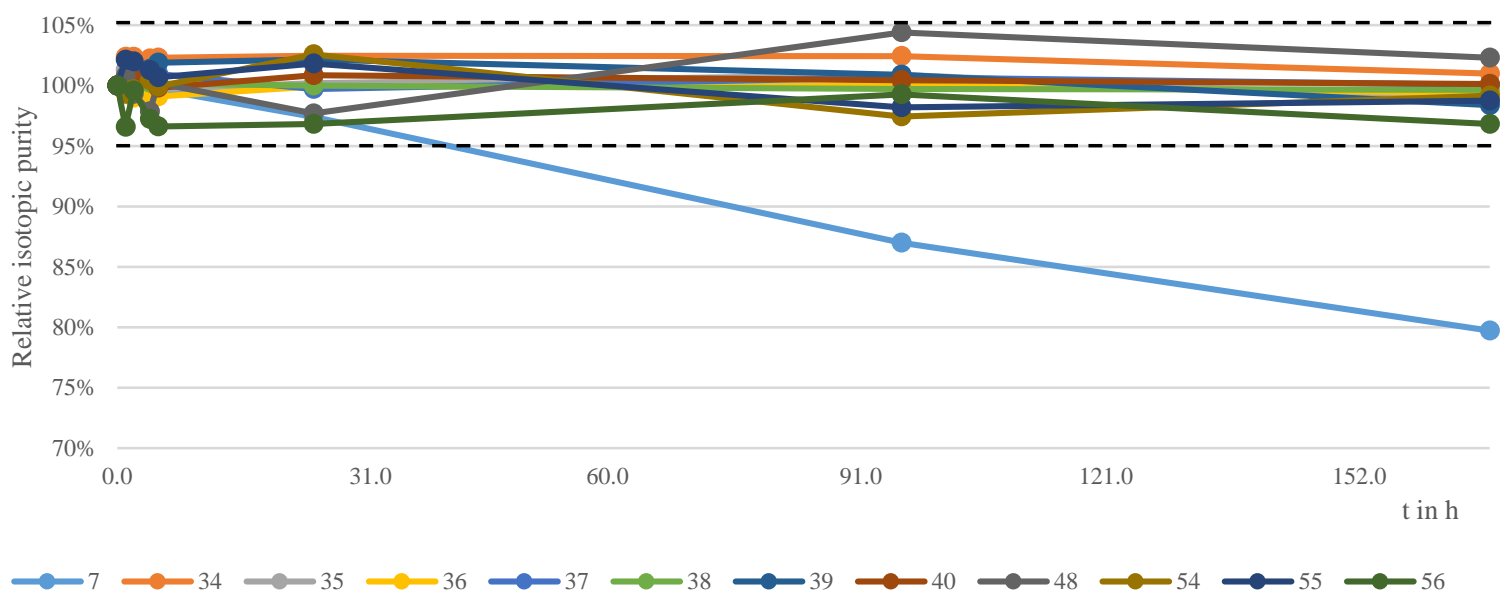

Figure S4: Kinetic stability assay in $50: 50 \mathrm{CH}_{3} \mathrm{CN}: \mathrm{H}_{2} \mathrm{O}+0.1 \%$ formic acid $(1.0 \mathrm{~mL}, 10 \mathrm{mM}, \mathrm{pH} \sim 2.7)$ at $10{ }^{\circ} \mathrm{C}$. Isotopic purity of the fully deuterated substrate was normalized to $100 \%$ at $\mathrm{t}_{0}$.

Table S5: Kinetic stability assay in $50: 50 \mathrm{CH}_{3} \mathrm{CN}: \mathrm{H}_{2} \mathrm{O}+0.1 \%$ formic acid $(1.0 \mathrm{~mL}, 10 \mathrm{mM}, \mathrm{pH} \sim 2.7)$ at $10{ }^{\circ} \mathrm{C}$. Isotopic purity of the fully deuterated substrate was normalized to $100 \%$ at $\mathrm{t}_{0}$.

\begin{tabular}{|r|r|r|r|r|r|r|r|r|r|r|r|r|r|r|r|r|r|}
\hline $\mathbf{t i n} \mathbf{h}$ & $\mathbf{7}$ & $\mathbf{3 4}$ & $\mathbf{3 5}$ & $\mathbf{3 6}$ & $\mathbf{3 7}$ & $\mathbf{3 8}$ & $\mathbf{3 9}$ & $\mathbf{4 8}$ & $\mathbf{5 4}$ & $\mathbf{5 5}$ \\
\hline $\mathbf{0 . 0}$ & $100.0 \%$ & $100.0 \%$ & $100.0 \%$ & $100.0 \%$ & $100.0 \%$ & $100.0 \%$ & $100.0 \%$ & $100.0 \%$ & $100.0 \%$ & $100.0 \%$ & $100.0 \%$ & $100.0 \%$ \\
\hline $\mathbf{1 . 3}$ & $99.5 \%$ & $102.4 \%$ & $101.3 \%$ & $99.9 \%$ & $100.0 \%$ & $100.0 \%$ & $100.1 \%$ & $100.1 \%$ & $100.8 \%$ & $99.3 \%$ & $102.1 \%$ & $96.6 \%$ \\
\hline $\mathbf{2 . 7}$ & $99.4 \%$ & $102.4 \%$ & $99.7 \%$ & $99.0 \%$ & $101.1 \%$ & $99.7 \%$ & $101.4 \%$ & $100.4 \%$ & $99.3 \%$ & $99.7 \%$ & $102.0 \%$ & $99.6 \%$ \\
\hline $\mathbf{4 . 0}$ & $99.2 \%$ & $102.2 \%$ & $100.9 \%$ & $99.3 \%$ & $100.3 \%$ & $100.2 \%$ & $101.4 \%$ & $101.2 \%$ & $97.9 \%$ & $100.4 \%$ & $101.3 \%$ & $97.2 \%$ \\
\hline $\mathbf{5 . 3}$ & $99.7 \%$ & $102.3 \%$ & $99.7 \%$ & $99.1 \%$ & $100.9 \%$ & $100.2 \%$ & $101.9 \%$ & $99.8 \%$ & $100.2 \%$ & $99.9 \%$ & $100.7 \%$ & $96.6 \%$ \\
\hline $\mathbf{2 4 . 0}$ & $97.4 \%$ & $102.5 \%$ & $100.2 \%$ & $99.9 \%$ & $99.7 \%$ & $100.0 \%$ & $102.2 \%$ & $100.8 \%$ & $97.7 \%$ & $102.6 \%$ & $101.8 \%$ & $96.8 \%$ \\
\hline $\mathbf{9 6 . 0}$ & $87.0 \%$ & $102.4 \%$ & $100.9 \%$ & $100.0 \%$ & $100.7 \%$ & $99.7 \%$ & $100.9 \%$ & $100.4 \%$ & $104.4 \%$ & $97.4 \%$ & $98.2 \%$ & $99.3 \%$ \\
\hline $\mathbf{1 6 8 . 0}$ & $79.7 \%$ & $101.0 \%$ & $98.8 \%$ & $99.4 \%$ & $100.1 \%$ & $99.6 \%$ & $98.4 \%$ & $100.1 \%$ & $102.3 \%$ & $99.2 \%$ & $98.7 \%$ & $96.8 \%$ \\
\hline
\end{tabular}




\section{${ }^{1} \mathrm{H}$ NMR SPECTRA OF THE LABELED AND UNLABELED COMPOUNDS}

(Deuterated product (red) stacked with non-deuterated substrate (blue))

1

$1 \mathrm{H}$ NMR (400 MHz, MeOD)

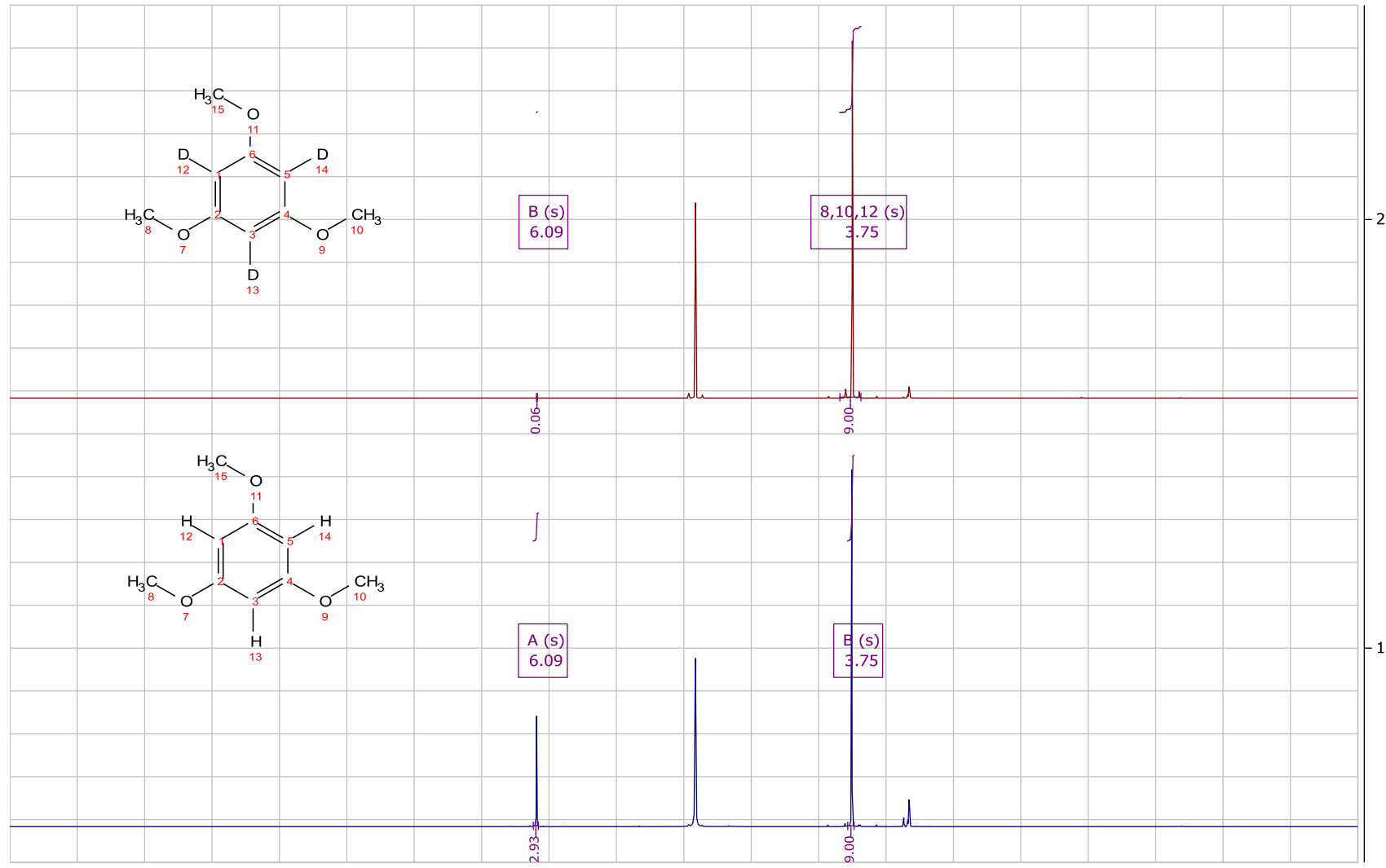

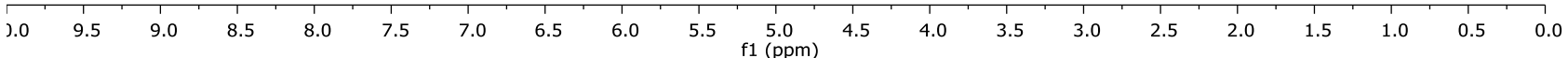


2

1H NMR (400 MHz, MeOD)

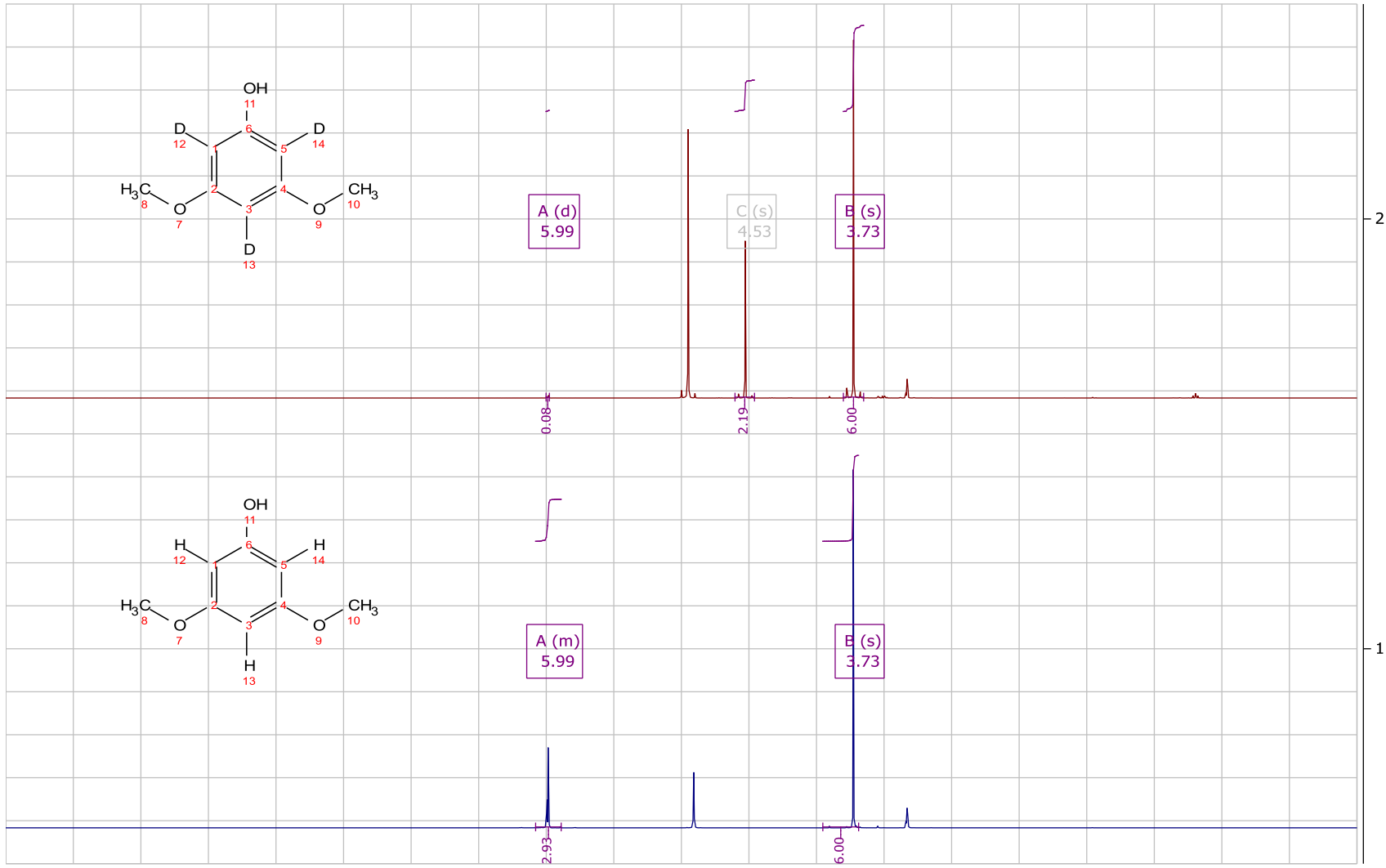

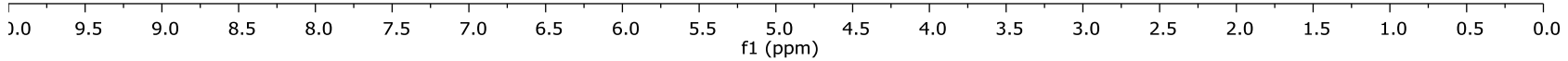


3

1H NMR (400 MHz, MeOD)

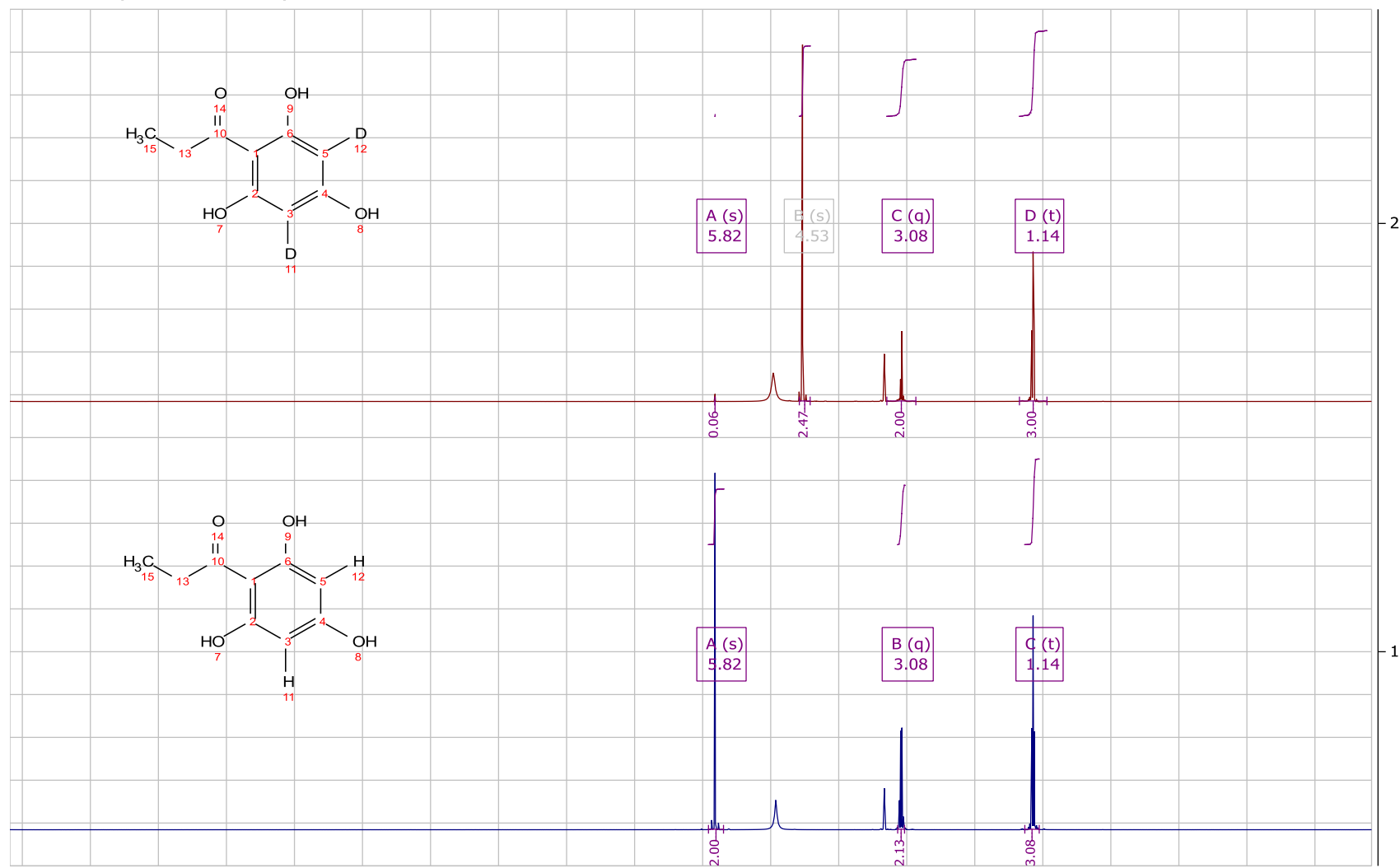


4

$1 \mathrm{H}$ NMR (400 MHz, MeOD)

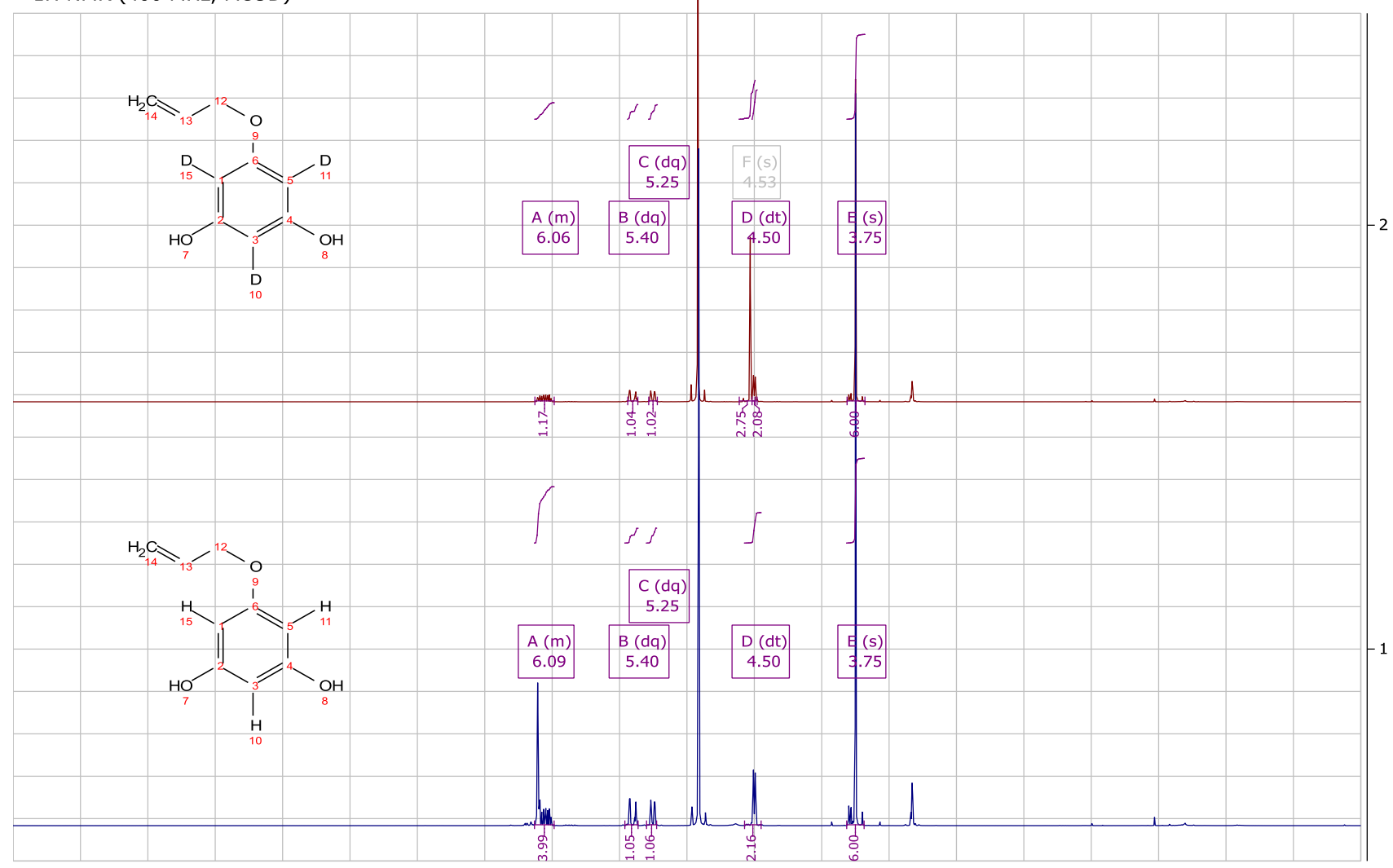

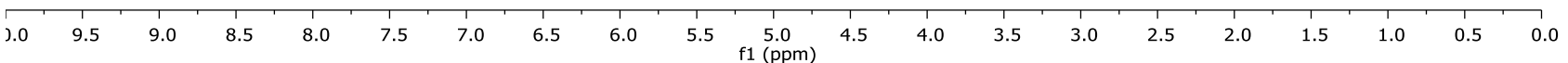


5

$1 \mathrm{H}$ NMR (400 MHz, MeOD)

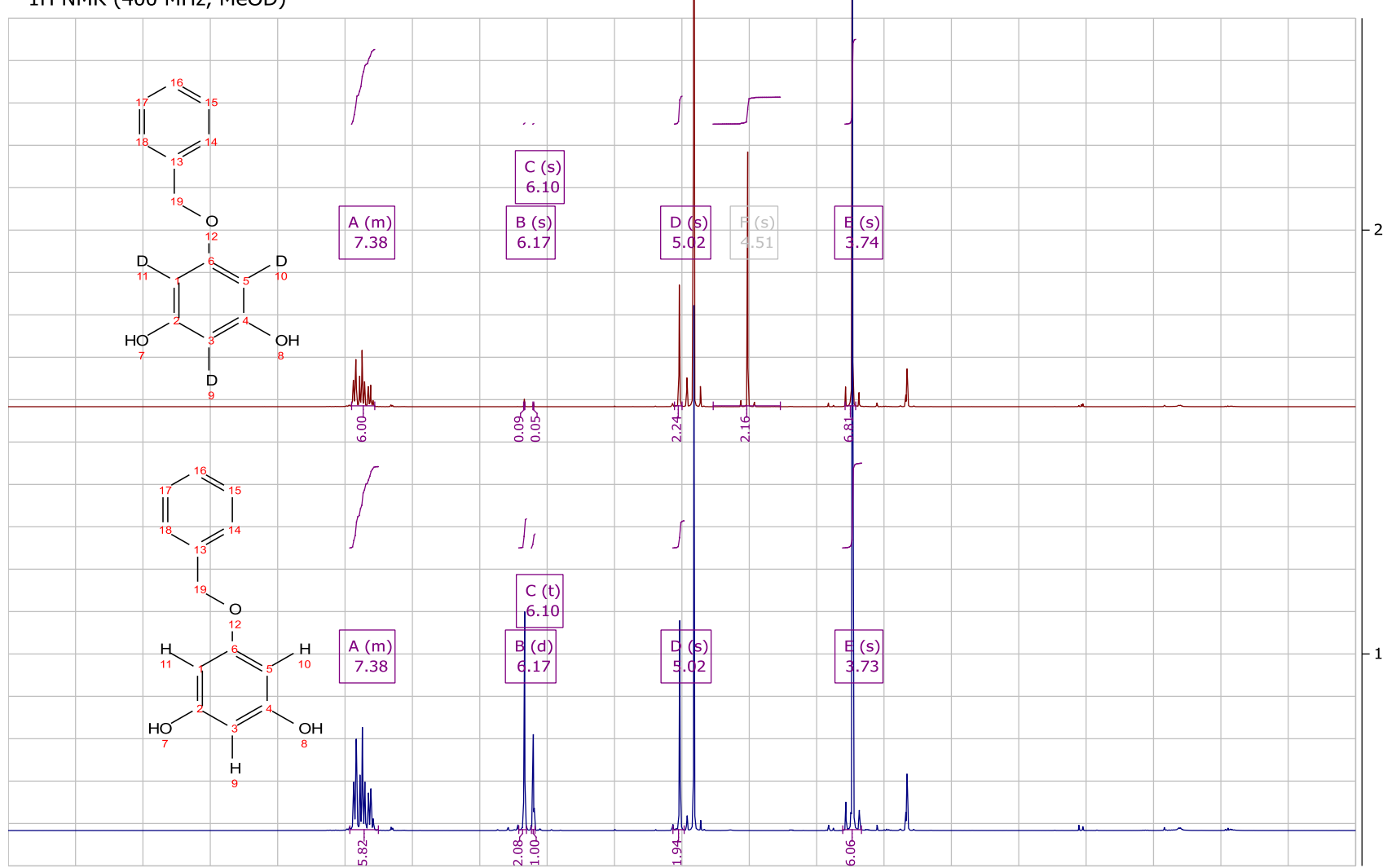

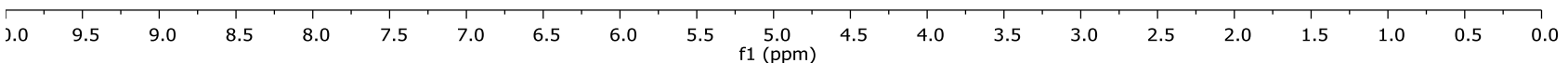


6
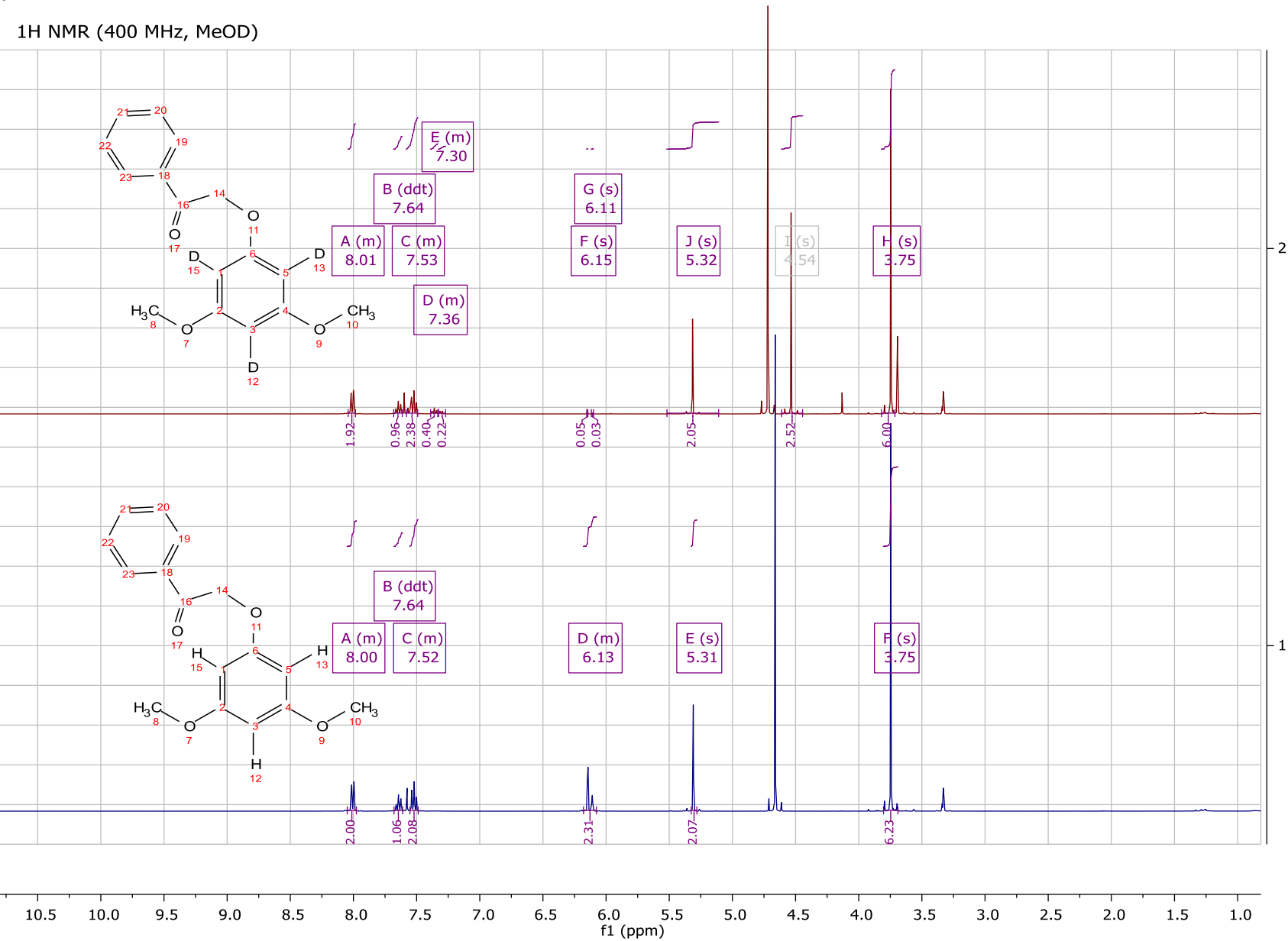
7

$1 \mathrm{H}$ NMR (400 MHz, MeOD)

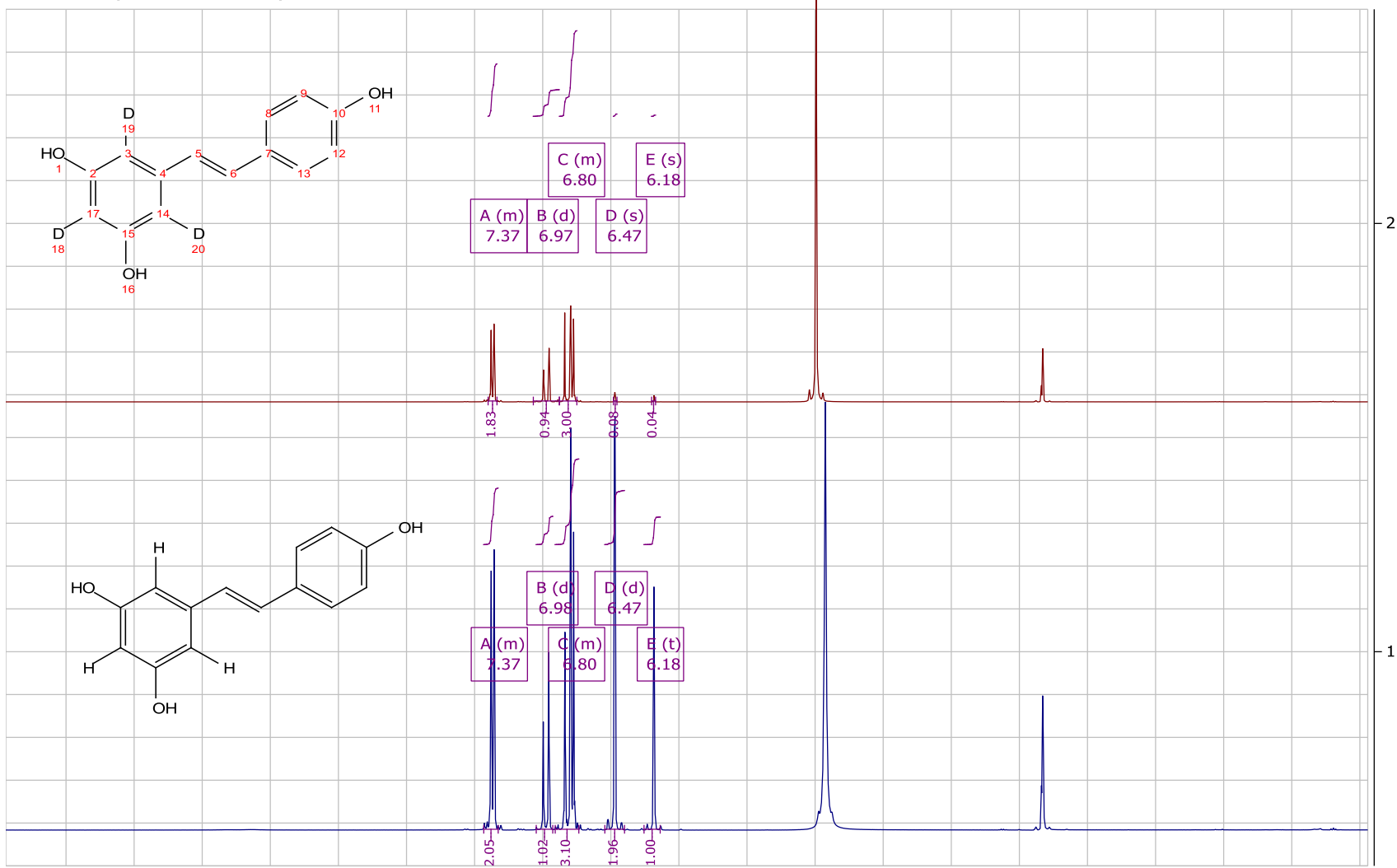

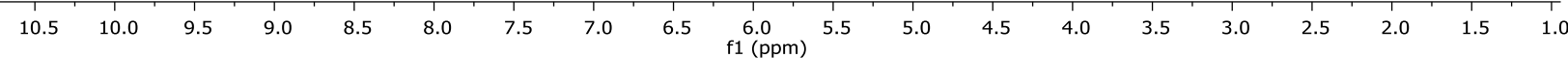


8

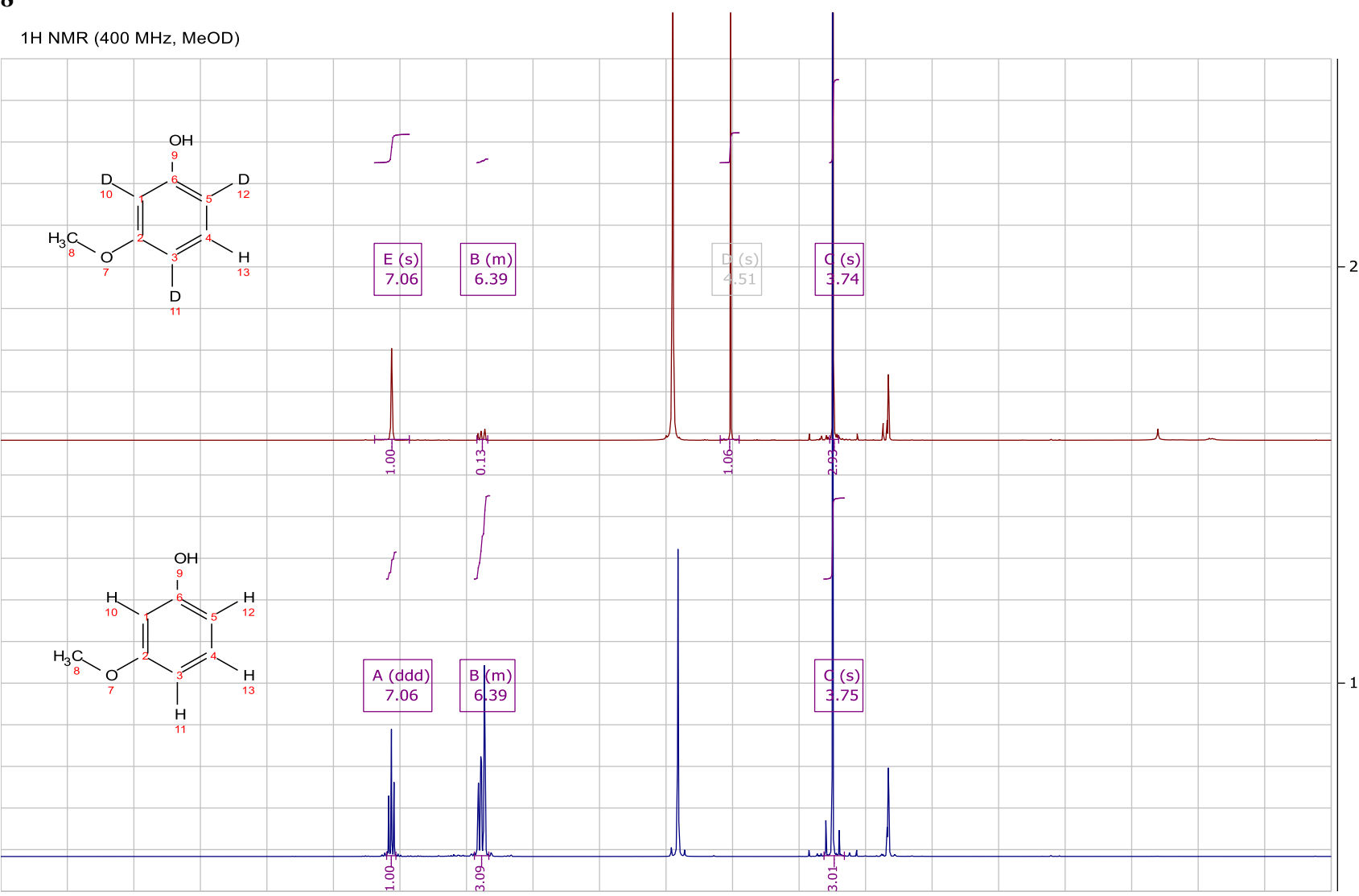

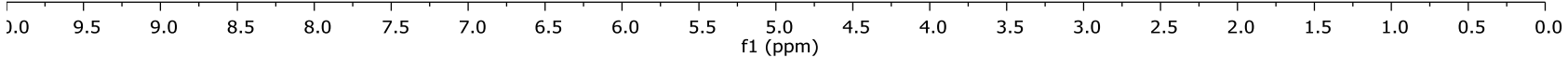


9

1H NMR (400 MHz, MeOD)

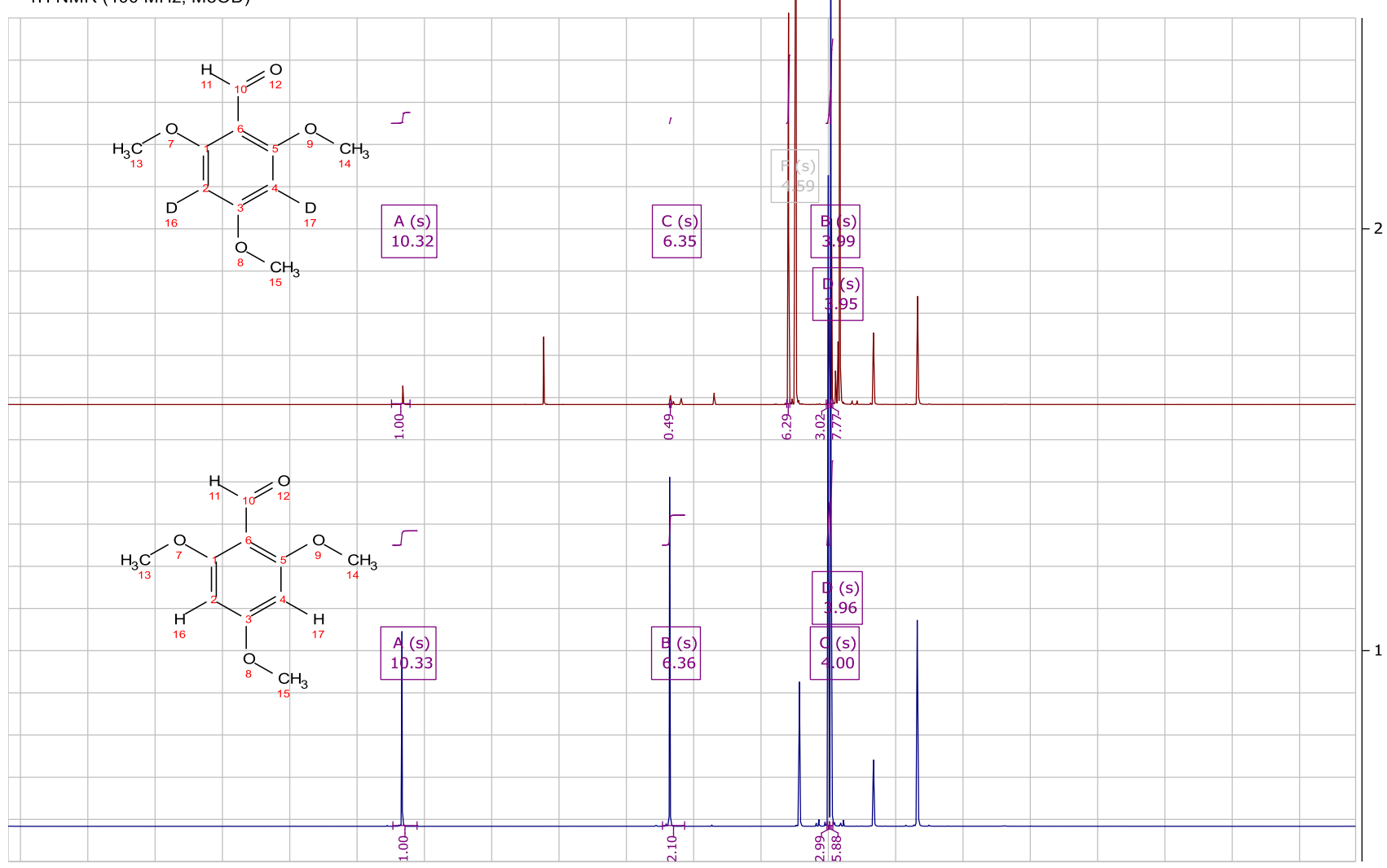

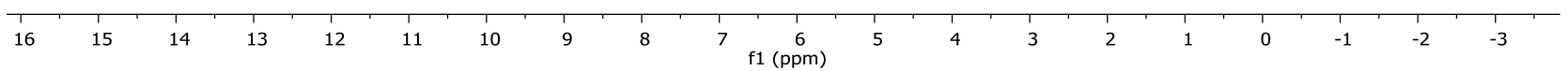


10

$1 \mathrm{H}$ NMR $(400 \mathrm{MHz}, \mathrm{MeOD})$

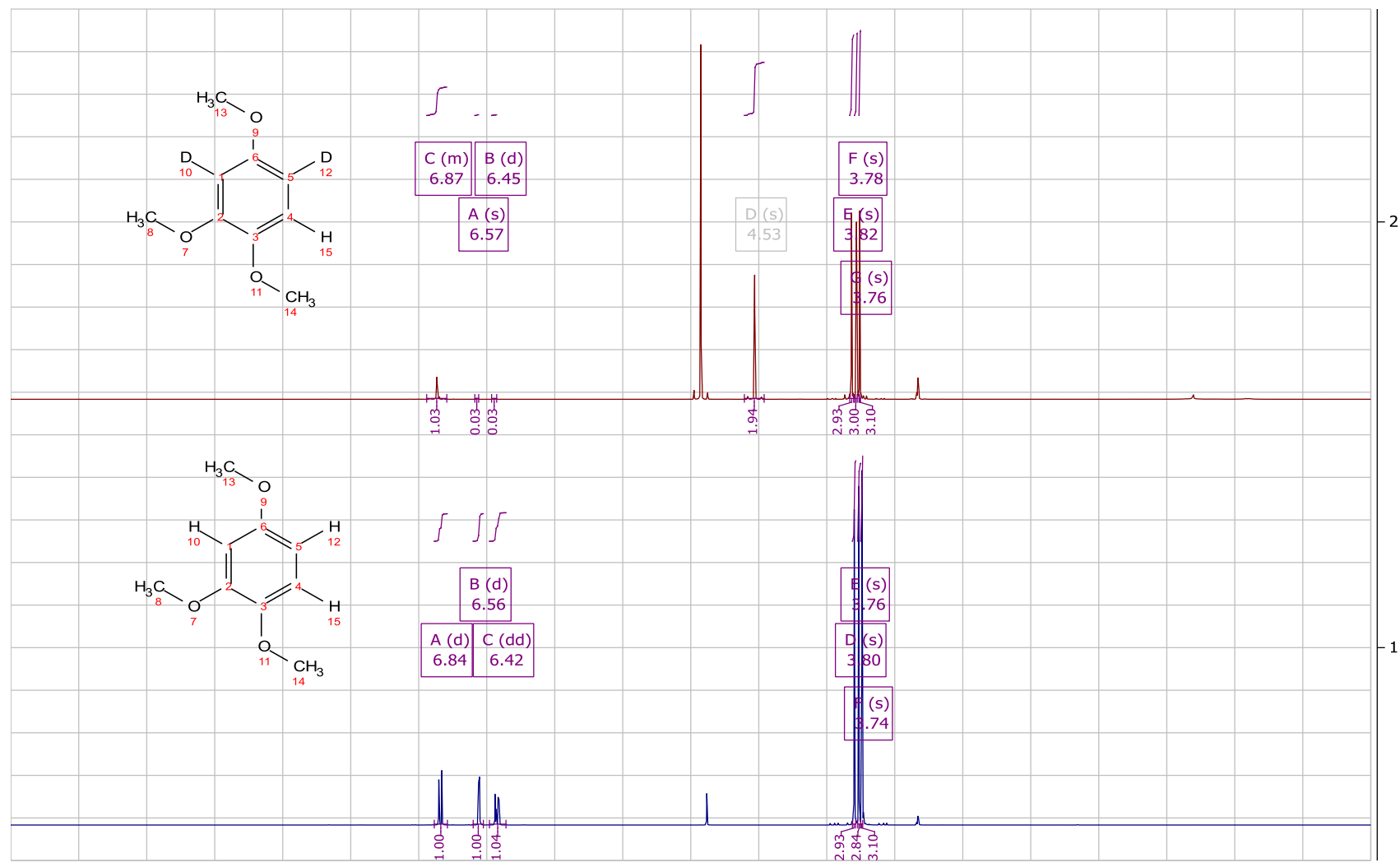

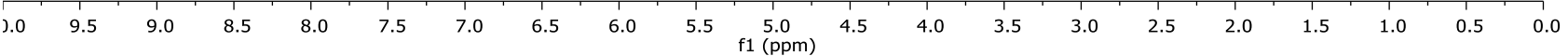


11

$1 \mathrm{H}$ NMR $(400 \mathrm{MHz}, \mathrm{MeOD})$

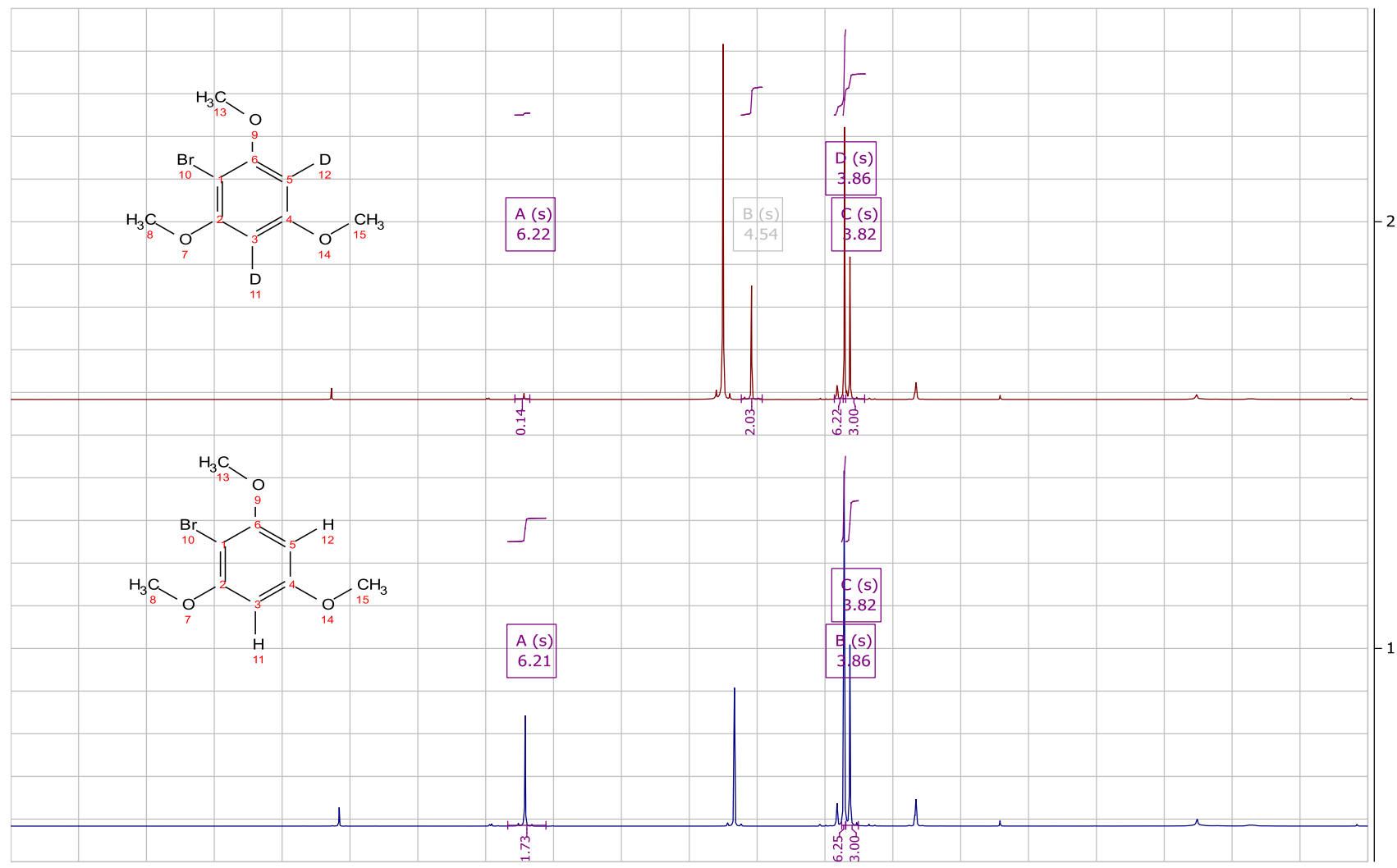

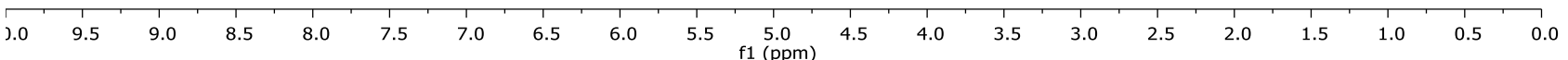


12

$1 \mathrm{H}$ NMR $(400 \mathrm{MHz}, \mathrm{MeOD})$

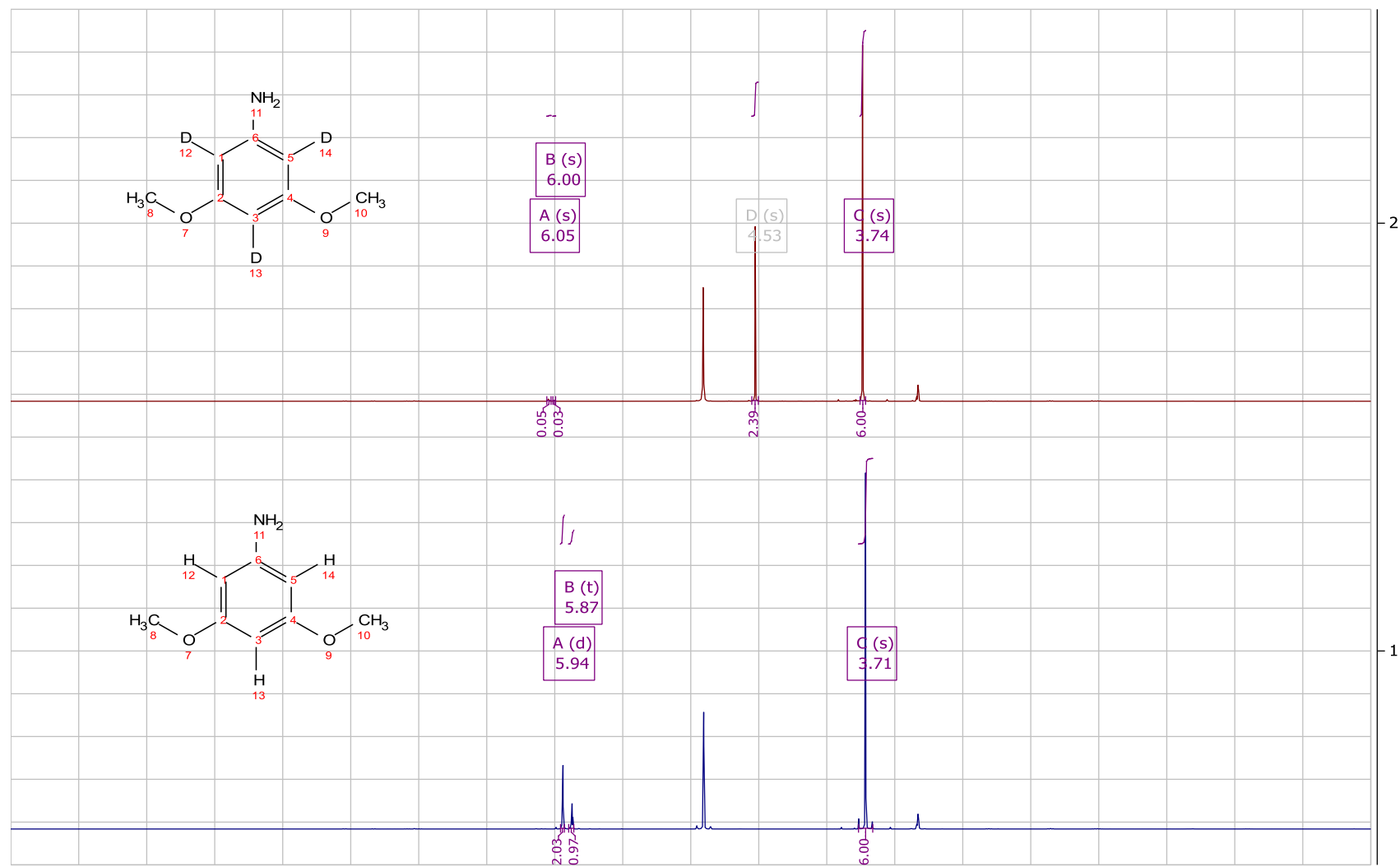

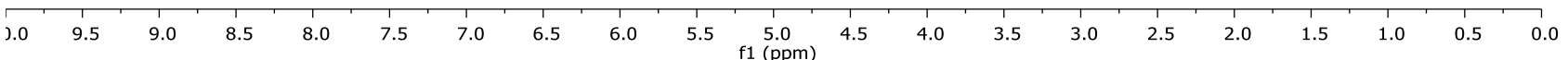


13

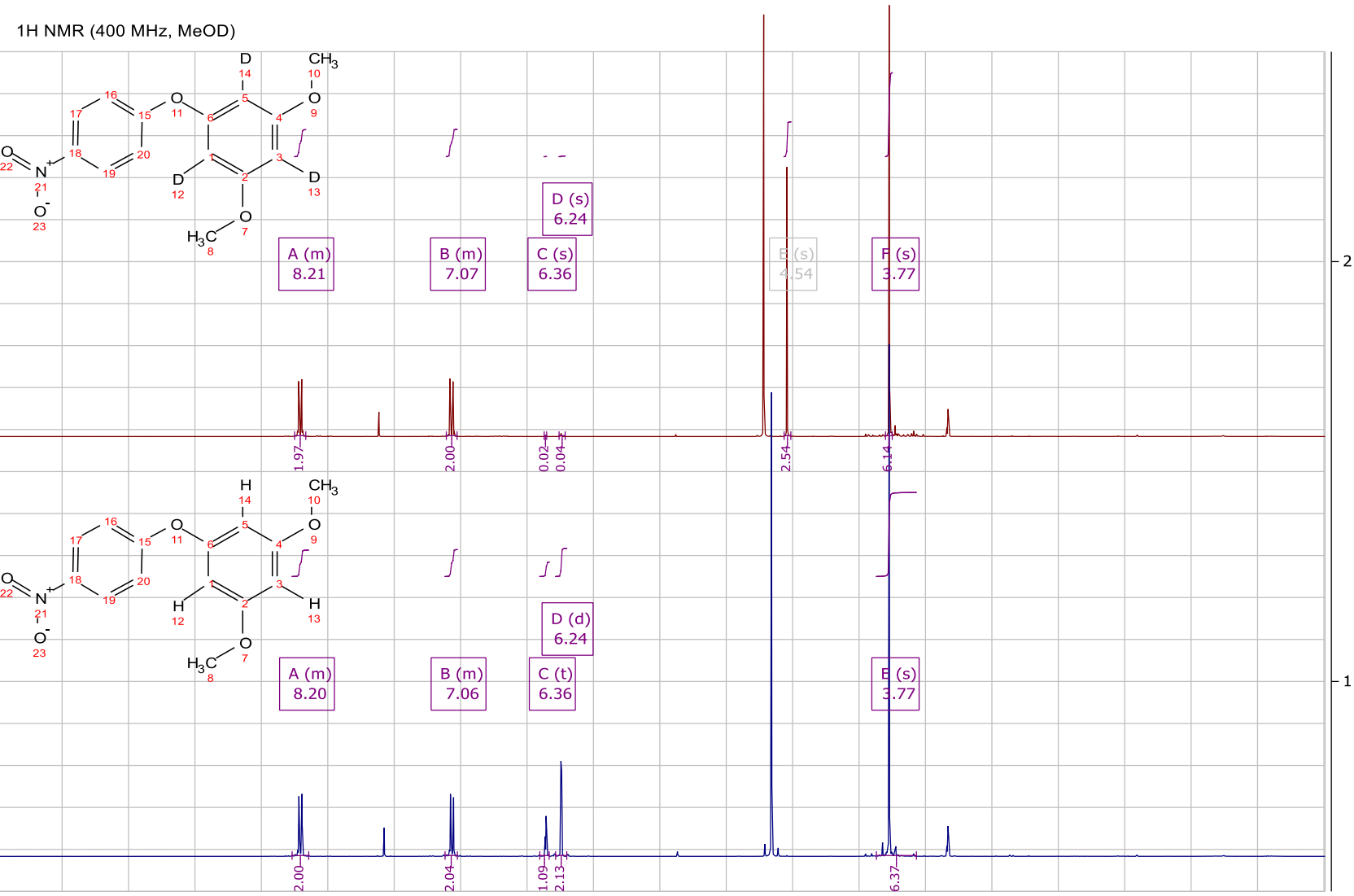

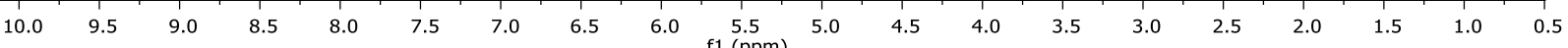


14

$1 \mathrm{H}$ NMR $(400 \mathrm{MHz}, \mathrm{MeOD})$

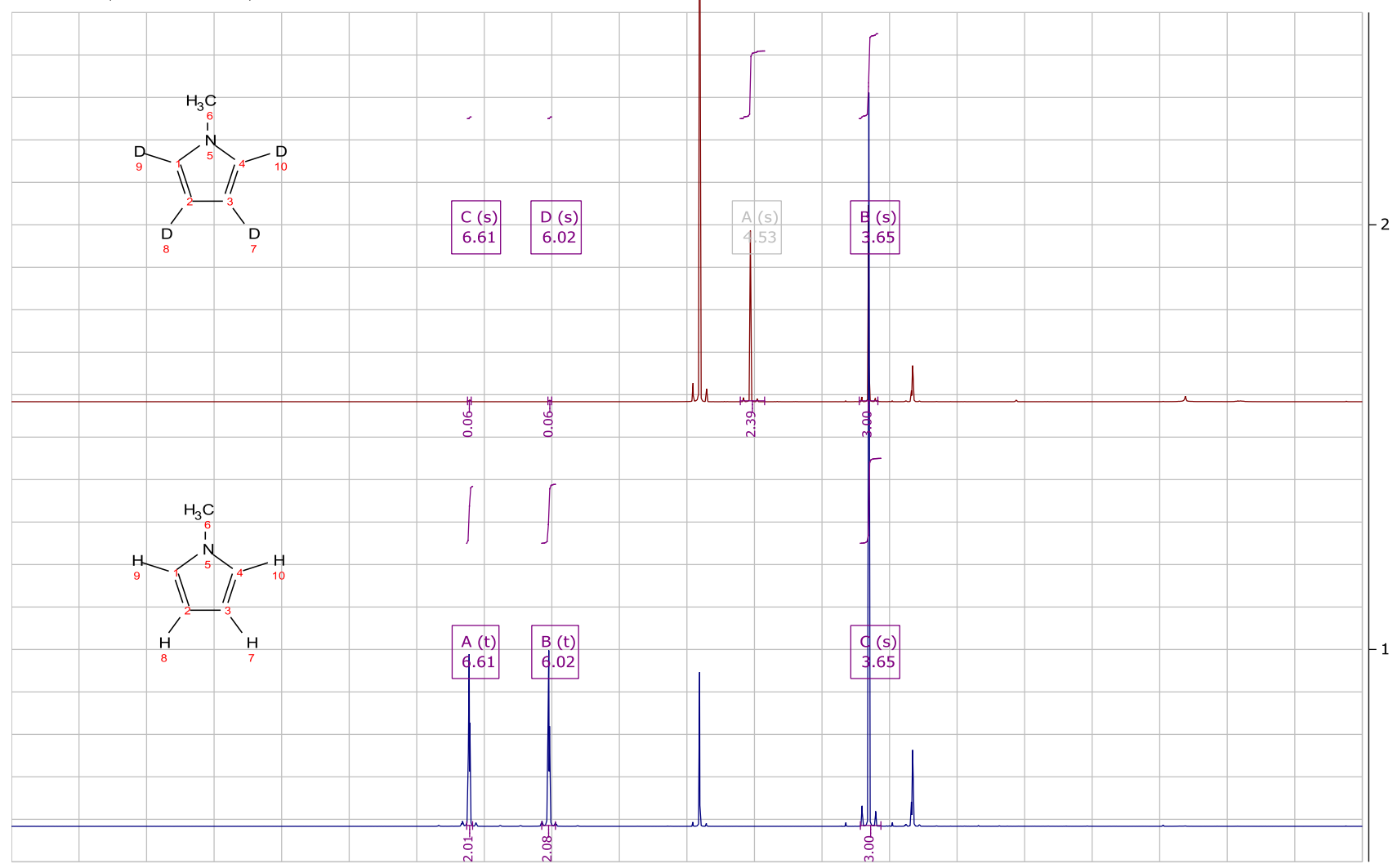

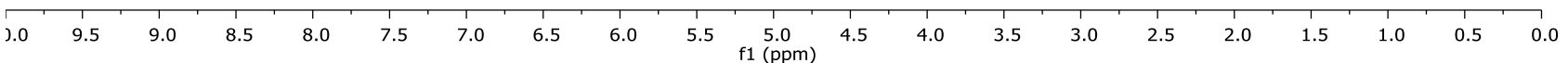


15

$1 \mathrm{H}$ NMR $(400 \mathrm{MHz}, \mathrm{MeOD})$

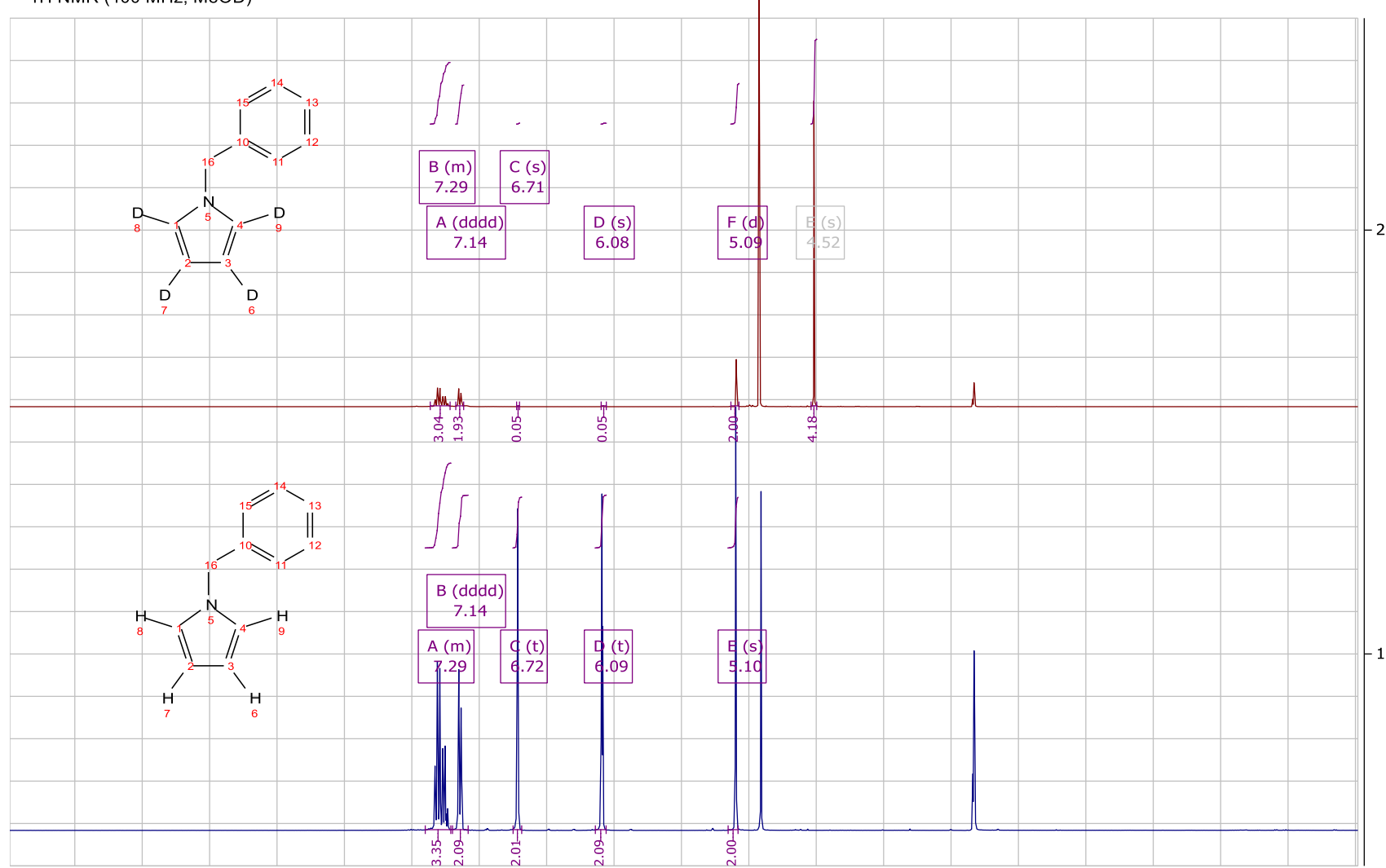

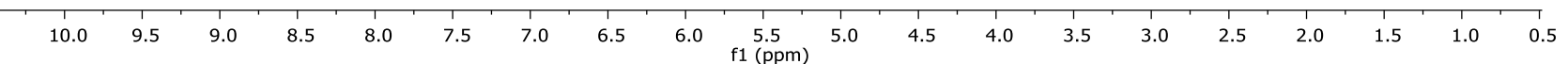


16

$1 \mathrm{H}$ NMR $(400 \mathrm{MHz}, \mathrm{MeOD})$

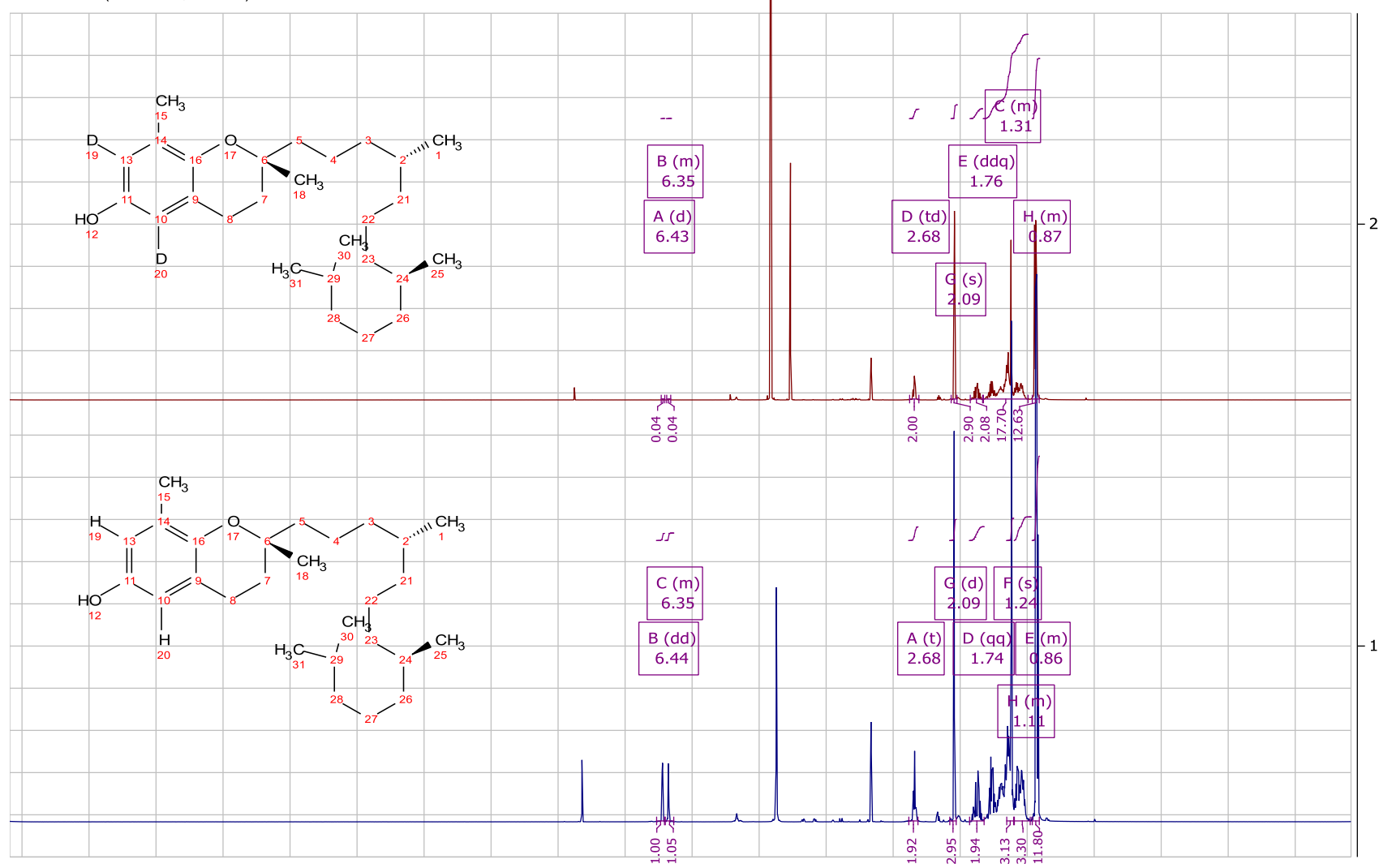

\begin{tabular}{lllllllllllllllllllll}
\hline 16 & 15 & 14 & 13 & 12 & 11 & 10 & 9 & 8 & 7 & $\begin{array}{c}6 \\
\mathrm{f} 1(\mathrm{ppm})\end{array}$ & 5 & 4 & 3 & 2 & 1 & 0 & -1 & -2 & -3 &
\end{tabular} 
17

$1 \mathrm{H}$ NMR (400 MHz, MeOD)

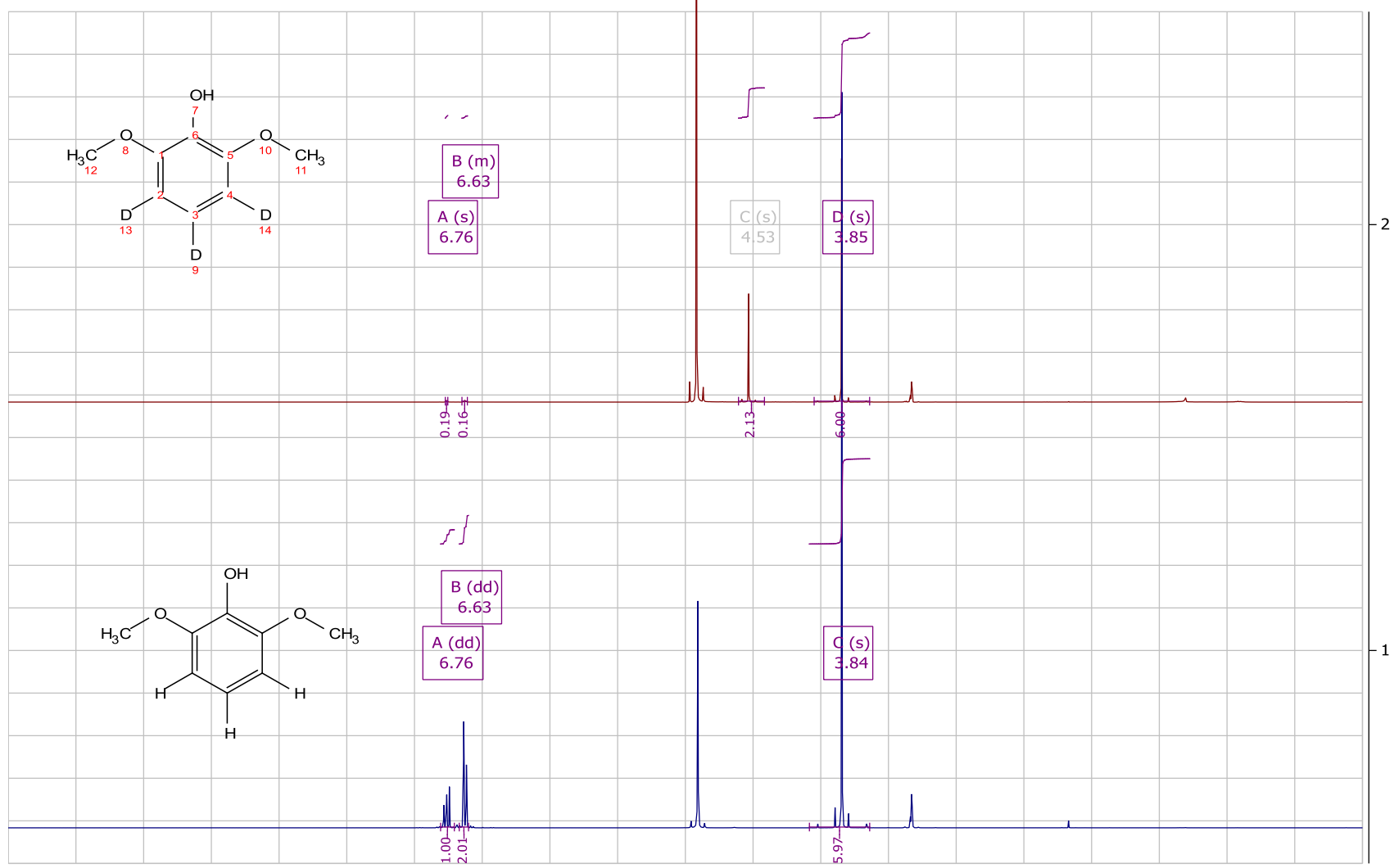

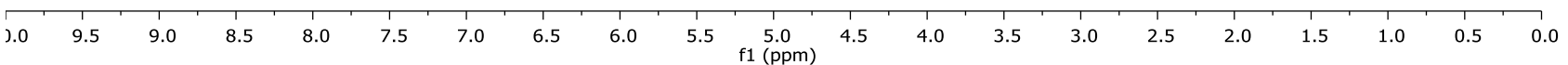


18

$1 \mathrm{H}$ NMR $(400 \mathrm{MHz}, \mathrm{MeOD})$

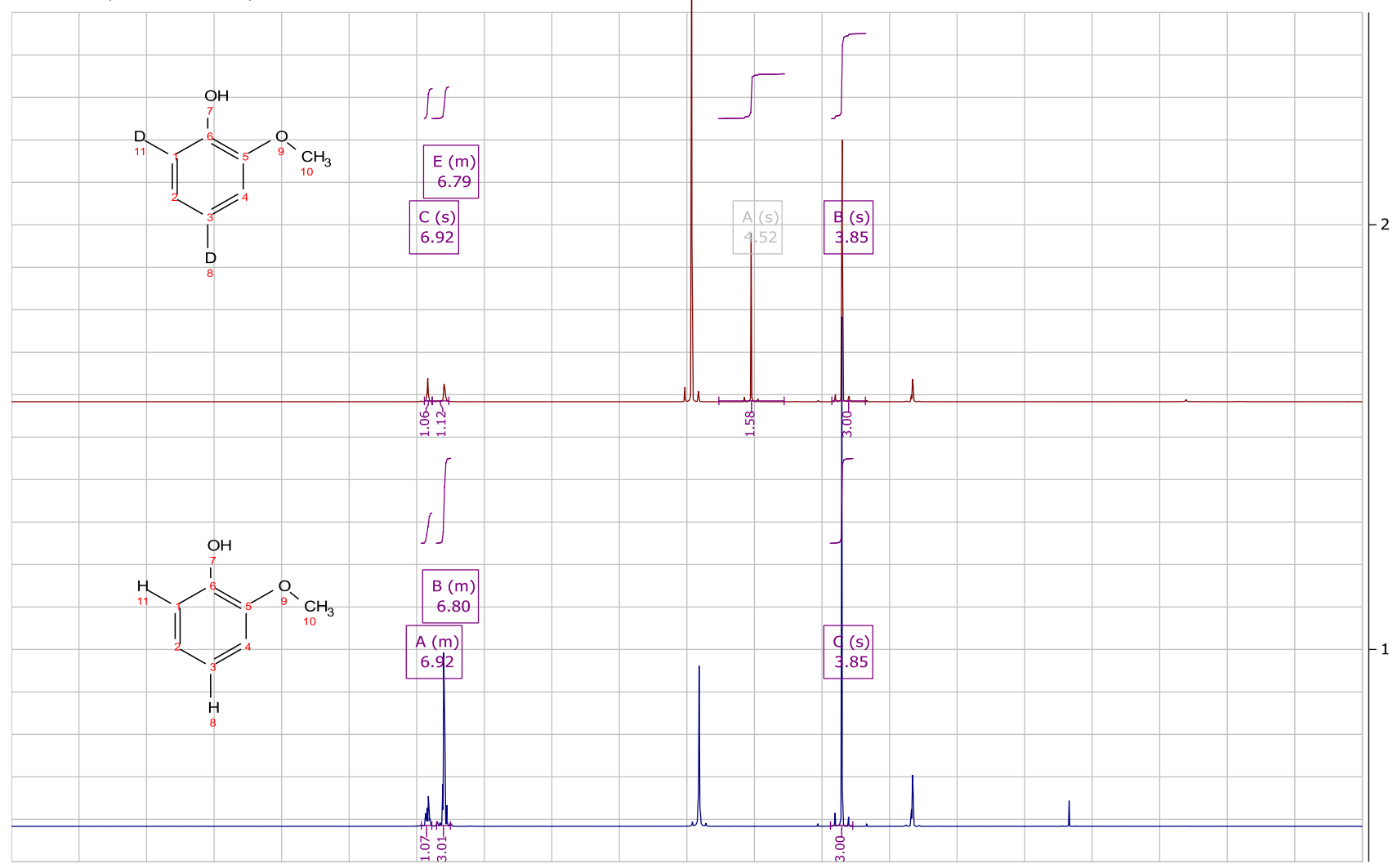

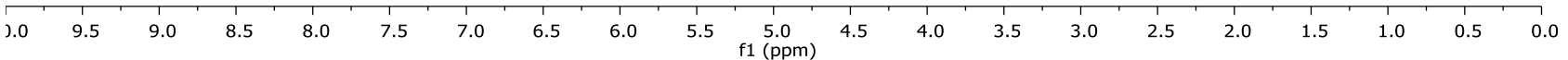


19

$1 \mathrm{H}$ NMR $(400 \mathrm{MHz}, \mathrm{MeOD})$

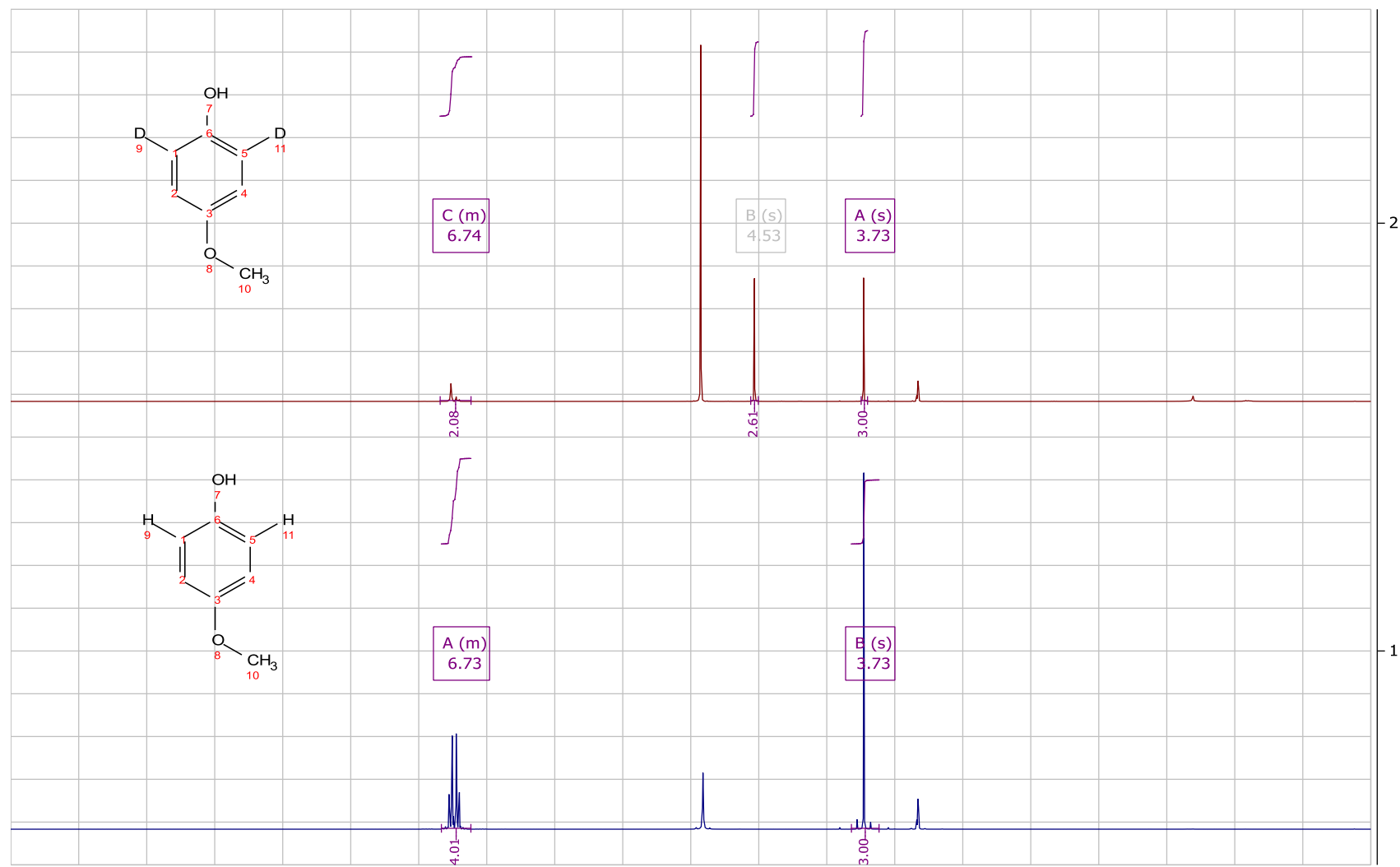

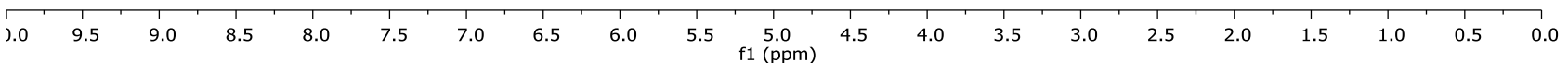


20

$1 \mathrm{H}$ NMR $(400 \mathrm{MHz}, \mathrm{MeOD})$

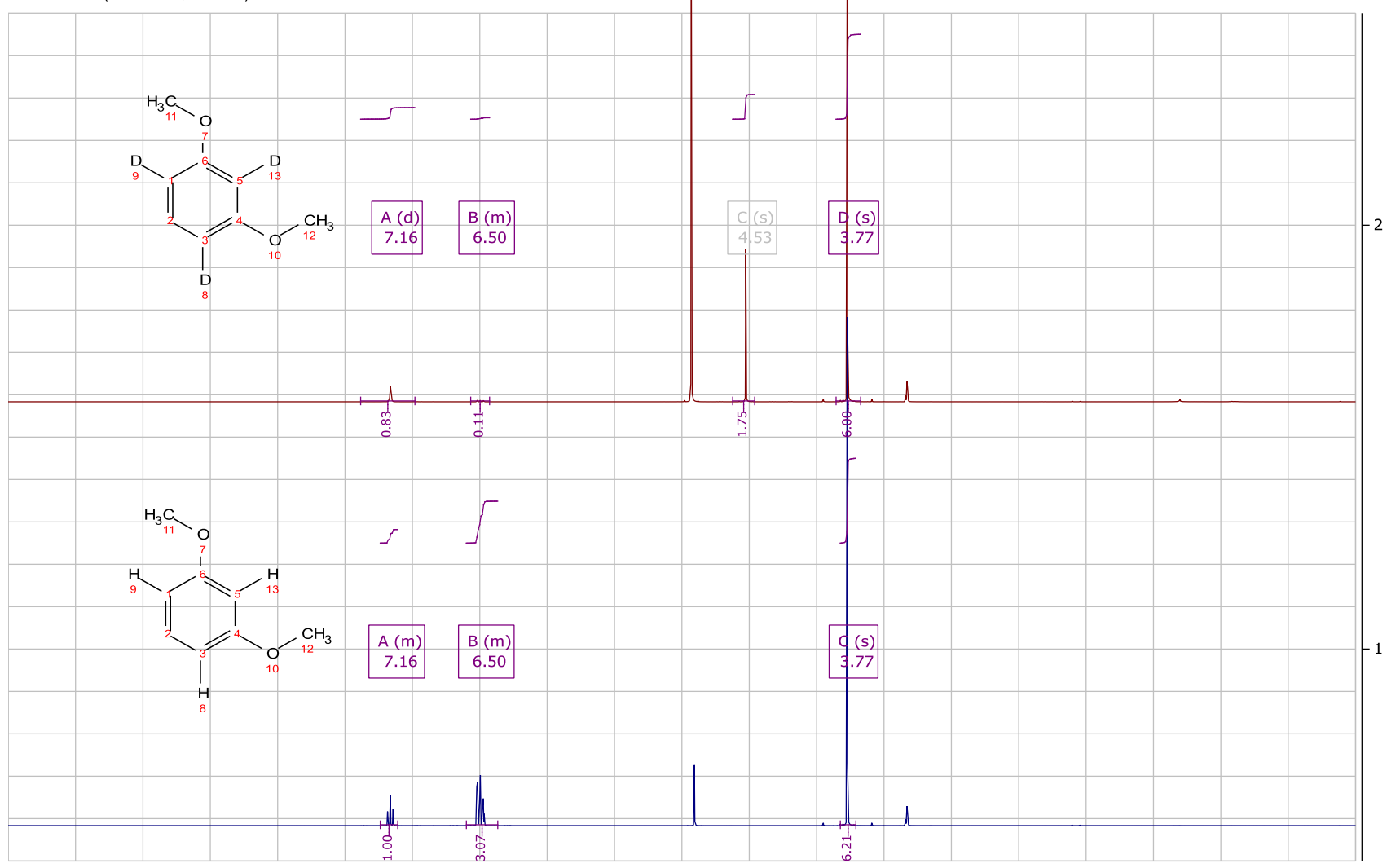

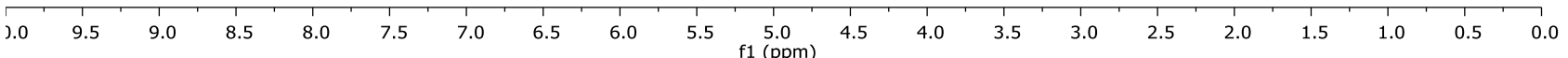


21

$1 \mathrm{H}$ NMR $(400 \mathrm{MHz}, \mathrm{MeOD})$

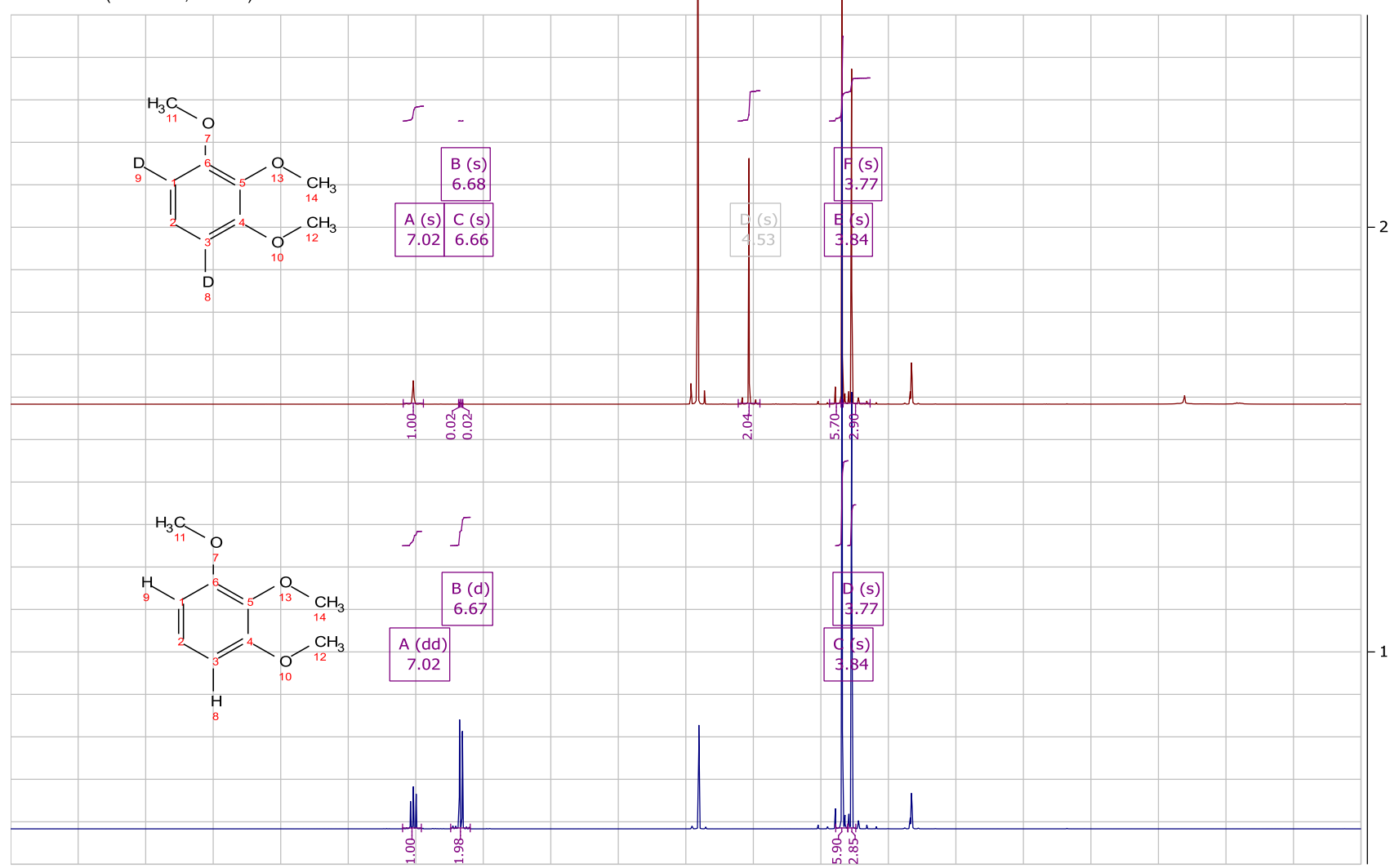

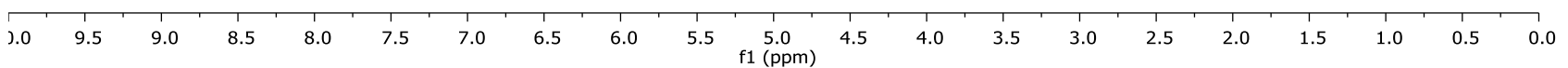


22

1H NMR (400 MHz, MeOD)
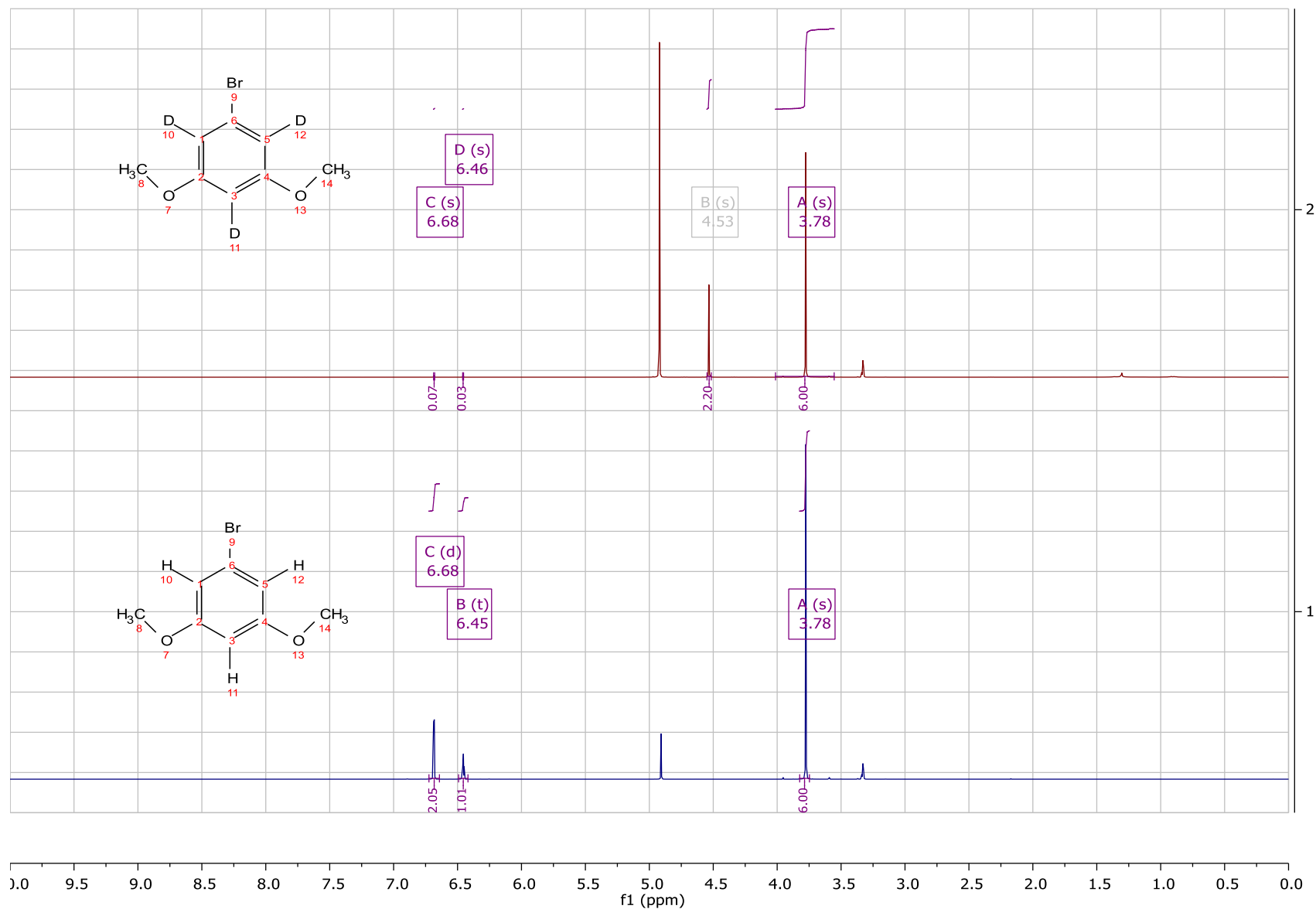
23

$1 \mathrm{H}$ NMR $(400 \mathrm{MHz}, \mathrm{MeOD})$

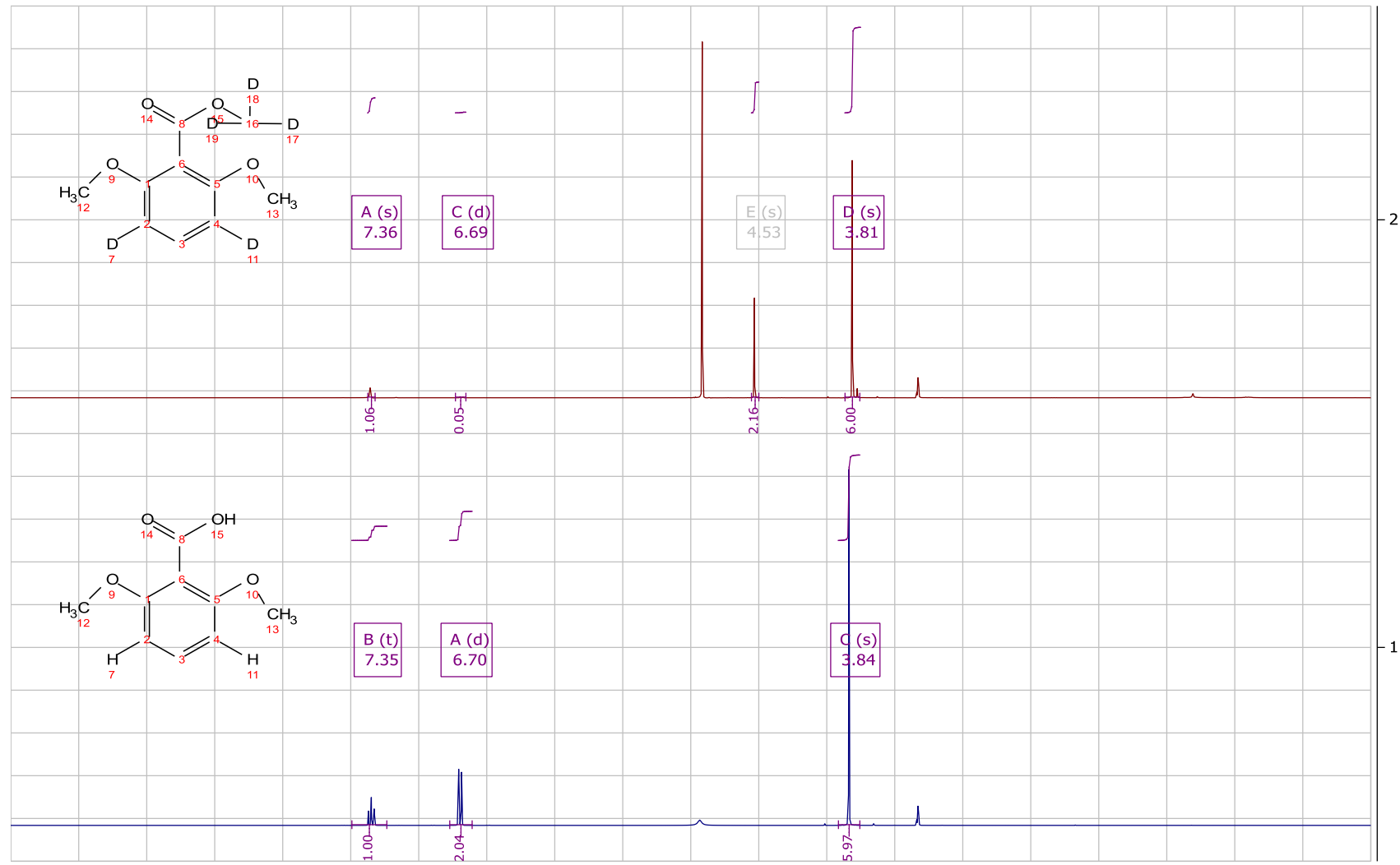

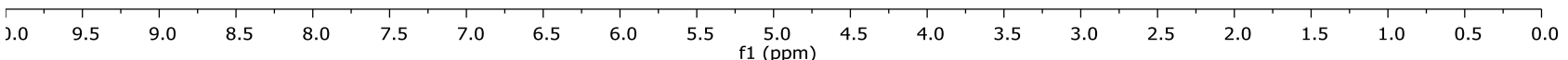


24

$1 \mathrm{H}$ NMR $(400 \mathrm{MHz}, \mathrm{MeOD})$

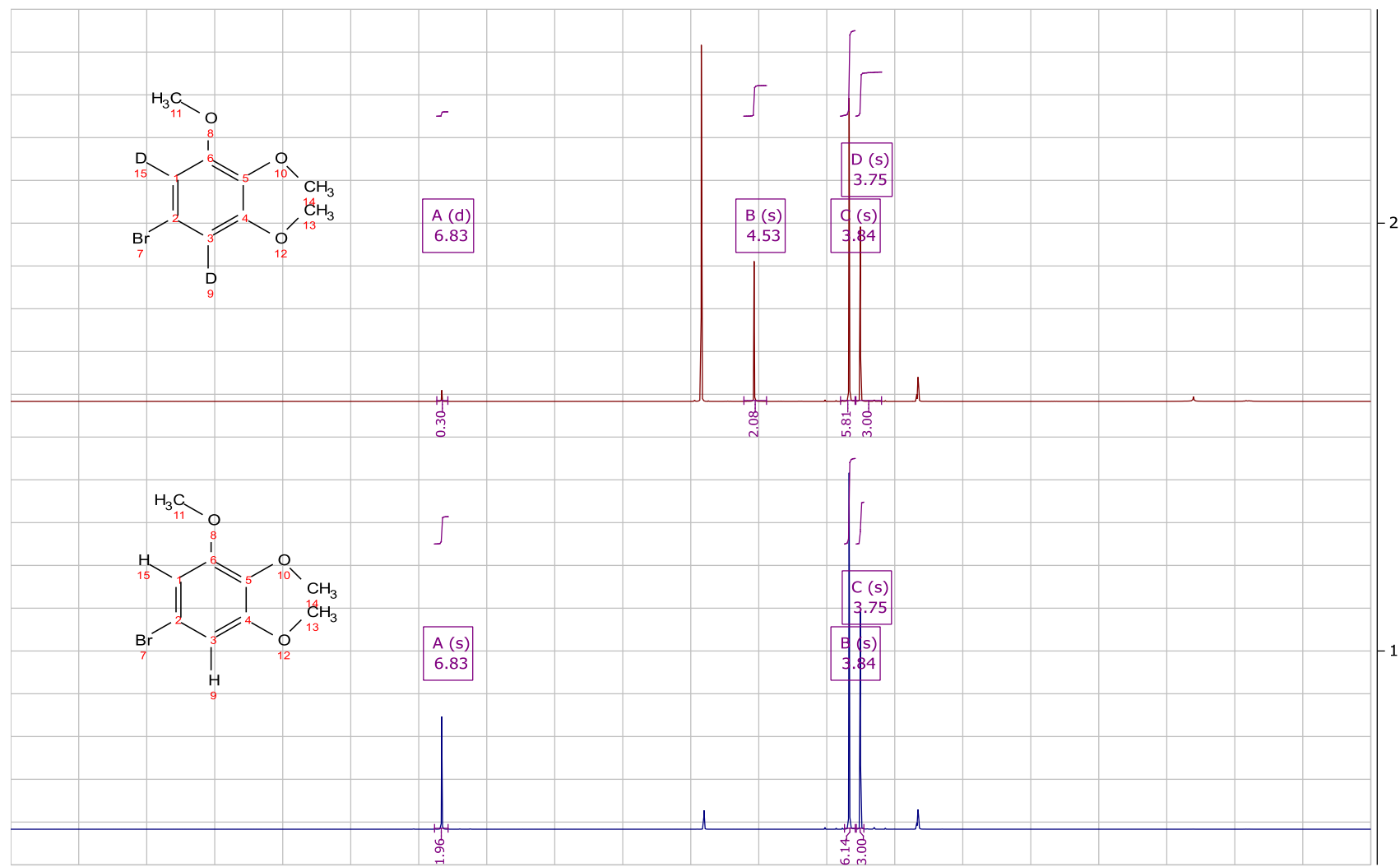

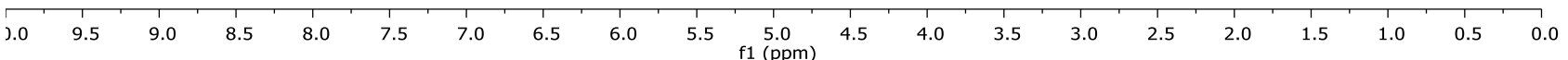


25

$1 \mathrm{H}$ NMR $(400 \mathrm{MHz}, \mathrm{MeOD})$

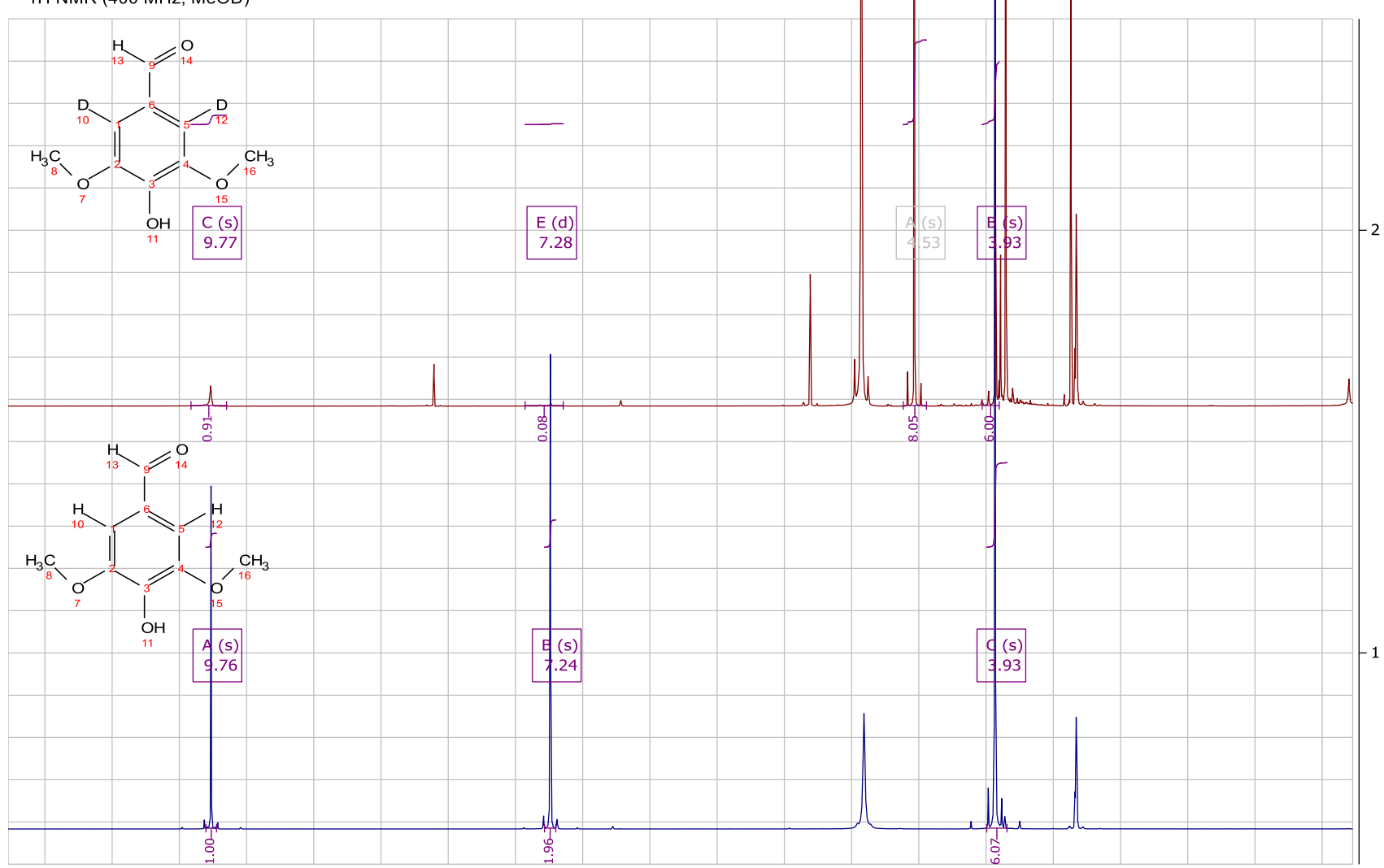

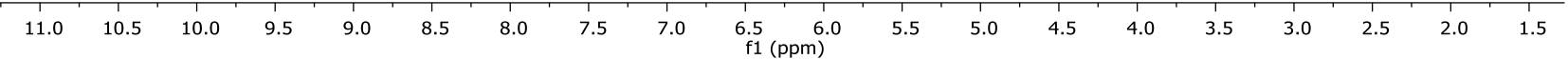




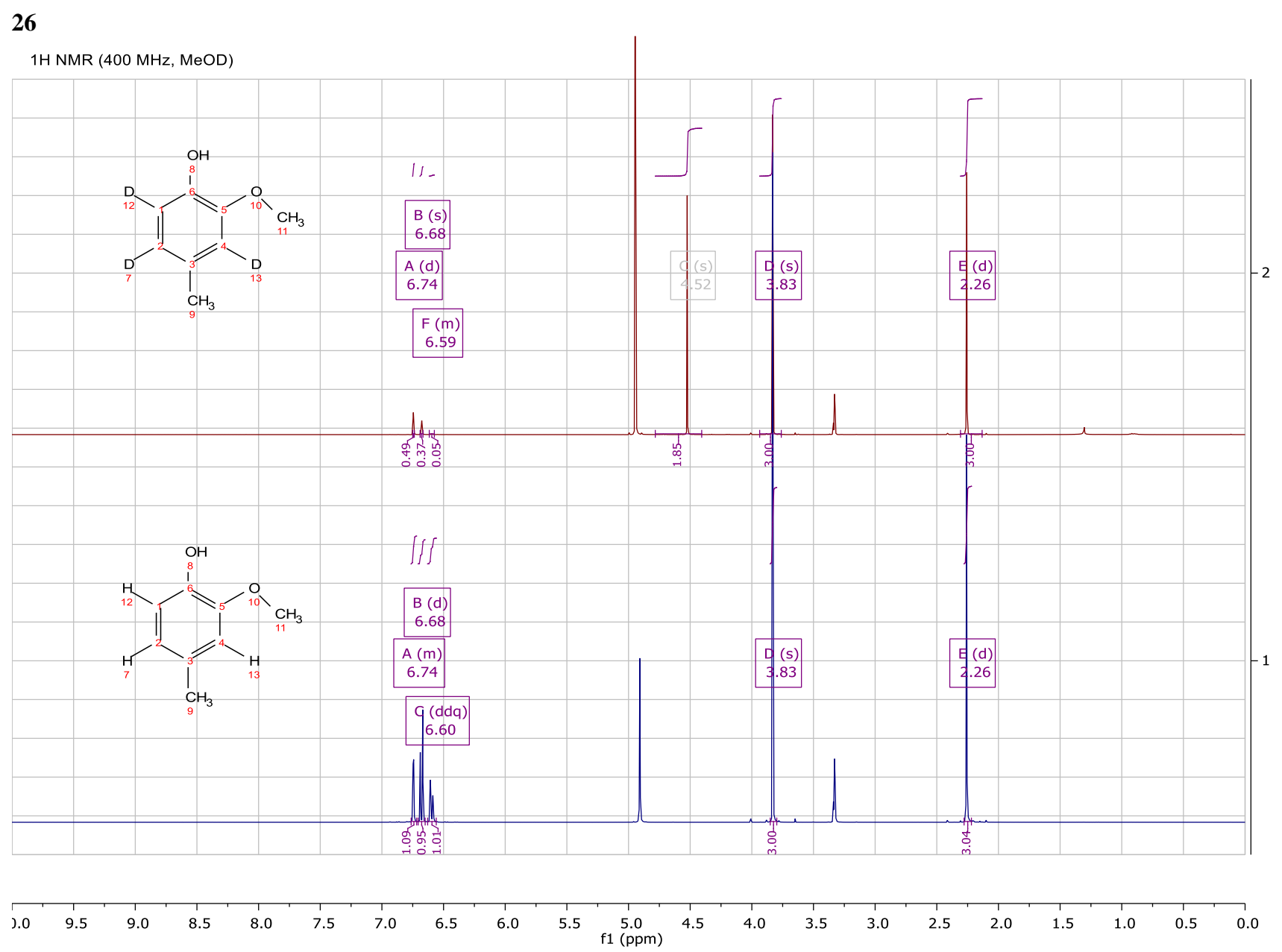


27

$1 \mathrm{H}$ NMR (400 MHz, MeOD)

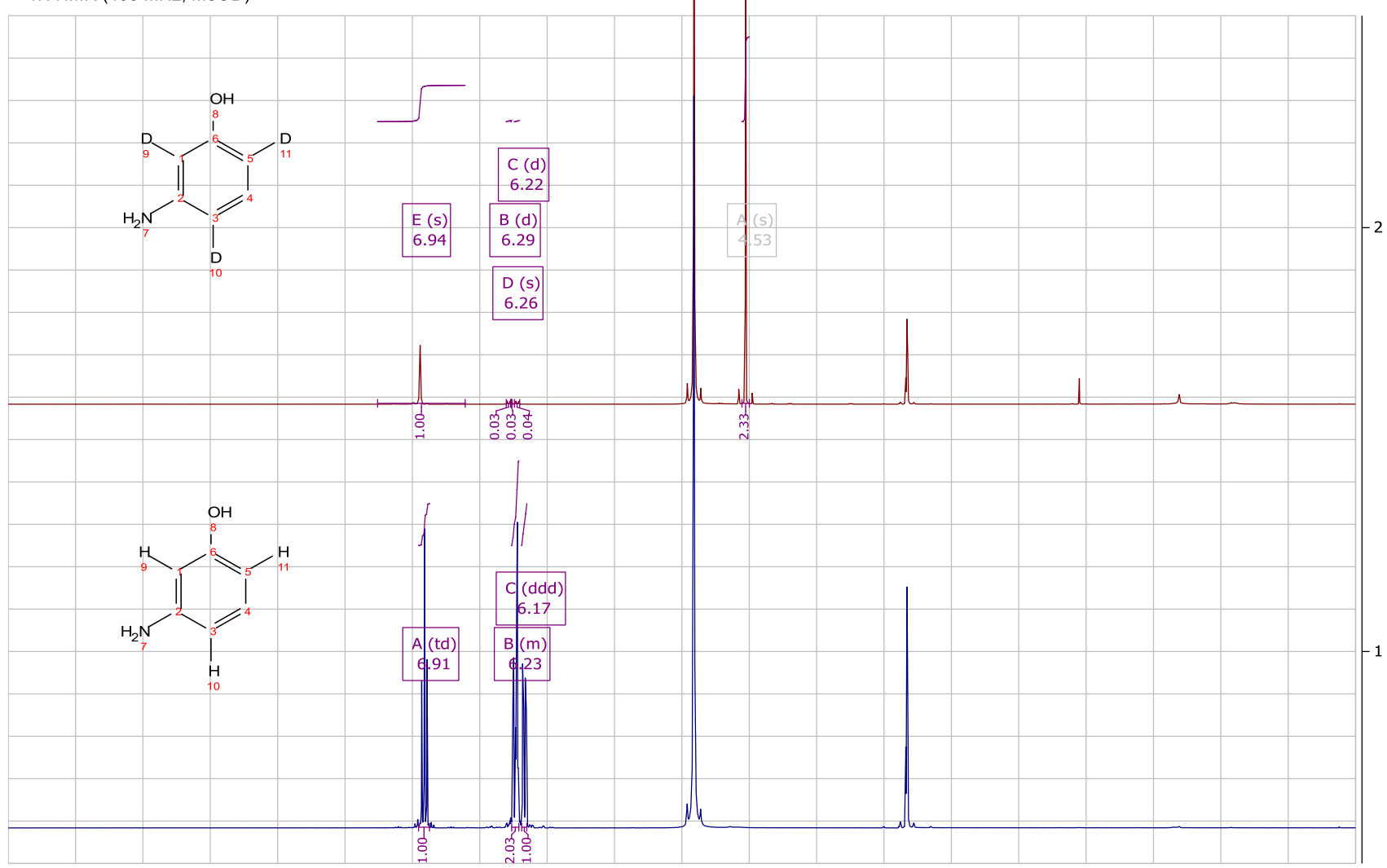

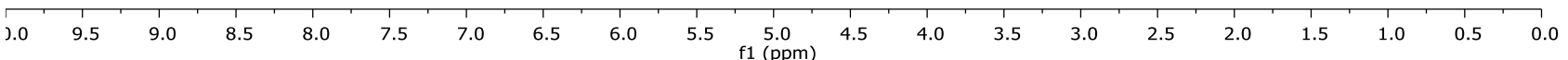


28

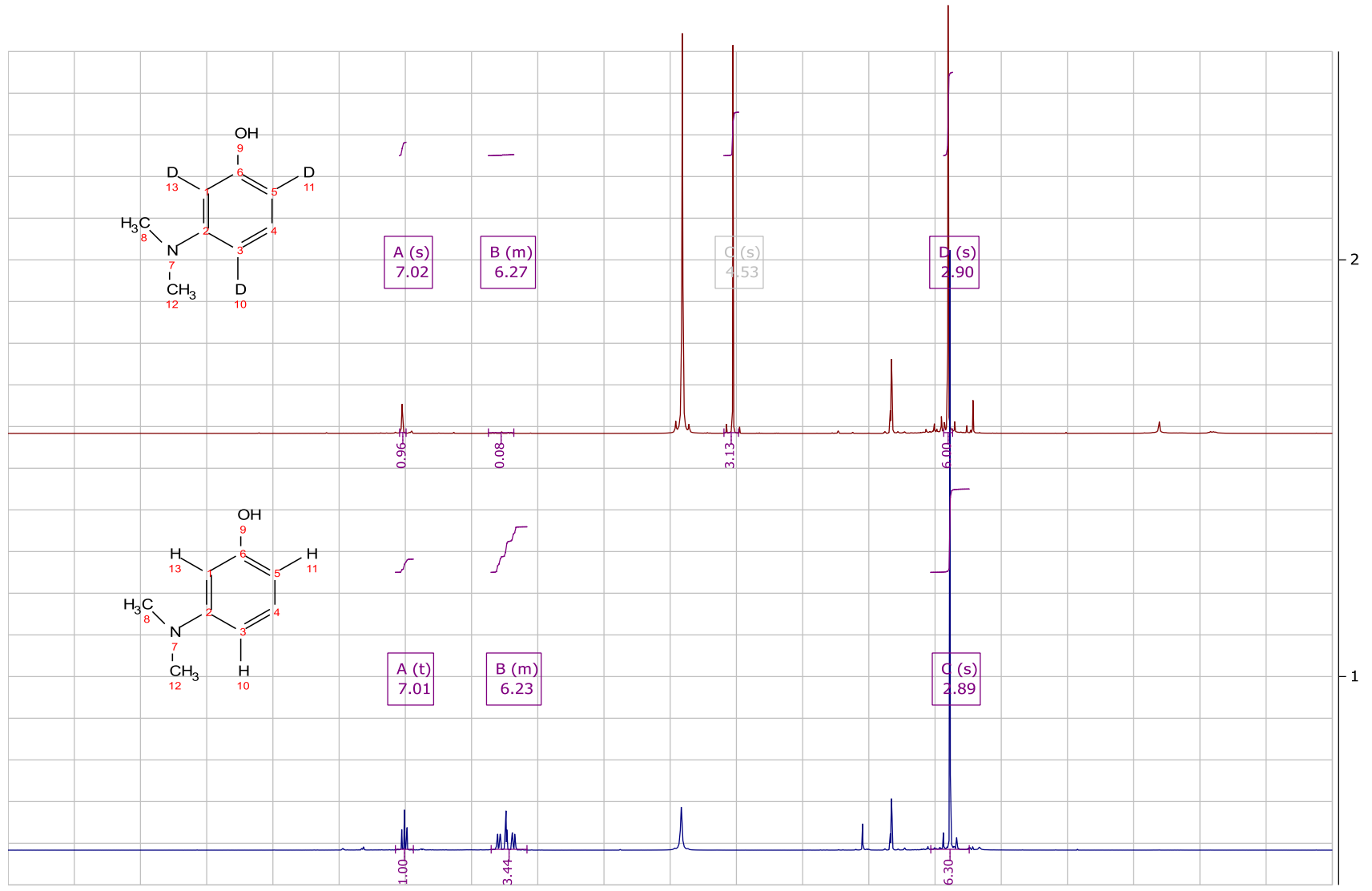

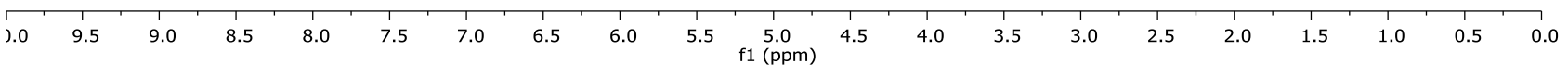


29

$1 \mathrm{H}$ NMR (400 MHz, MeOD)

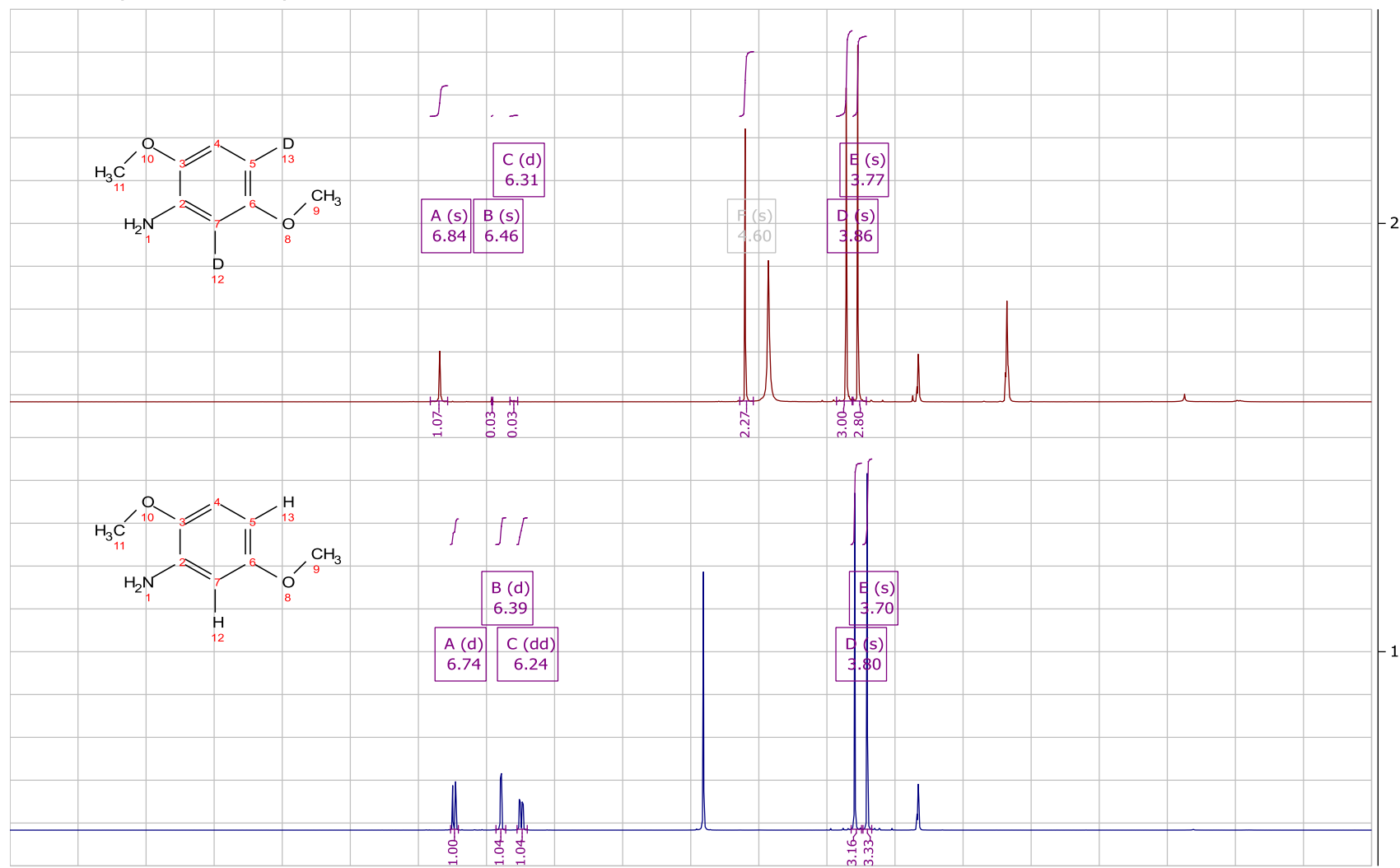

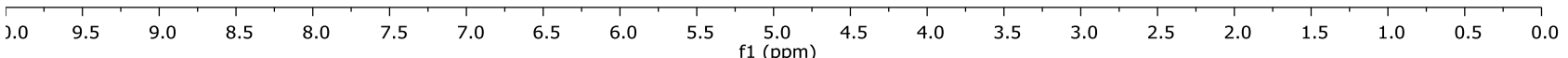


30

1H NMR (400 MHz, MeOD)

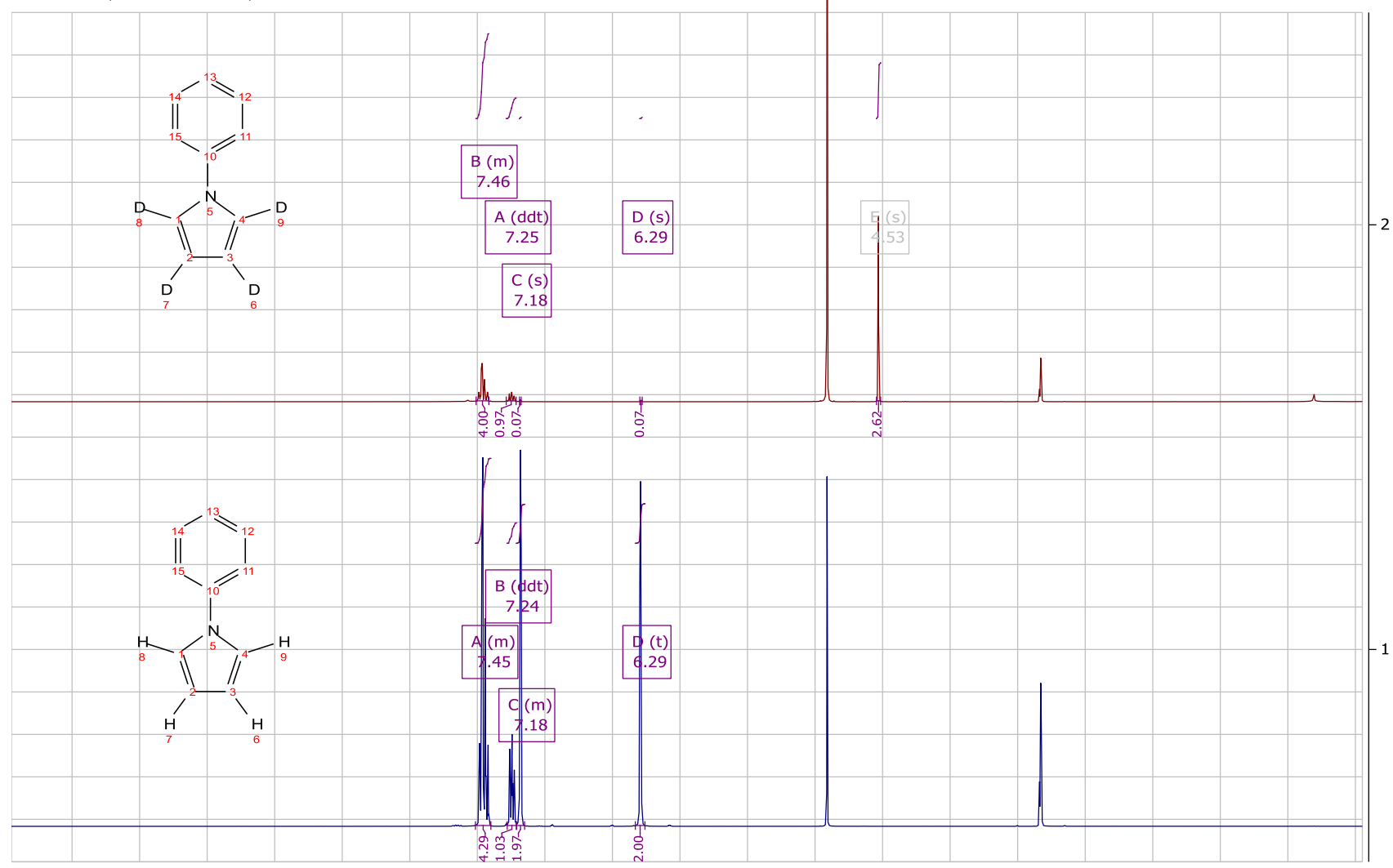

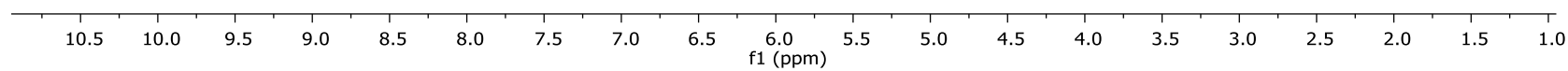


31

$1 \mathrm{H}$ NMR (400 MHz, MeOD)

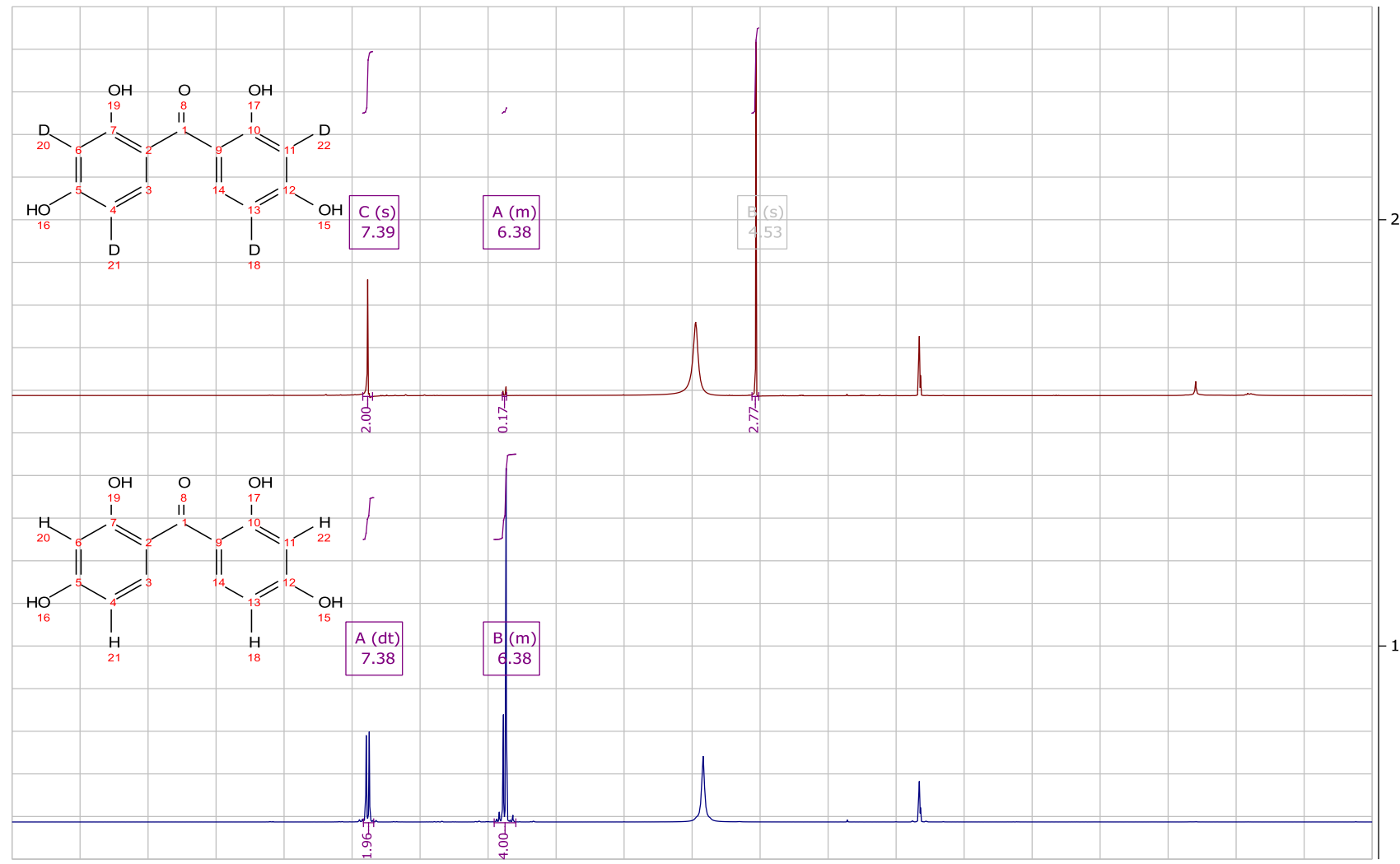

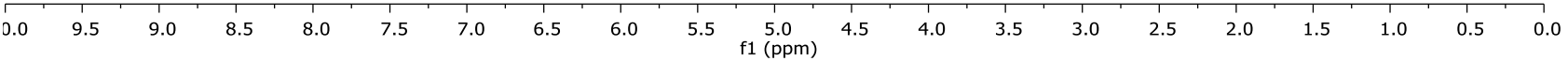


32

$1 \mathrm{H} \mathrm{NMR}(400 \mathrm{MHz}, \mathrm{MeOD})$

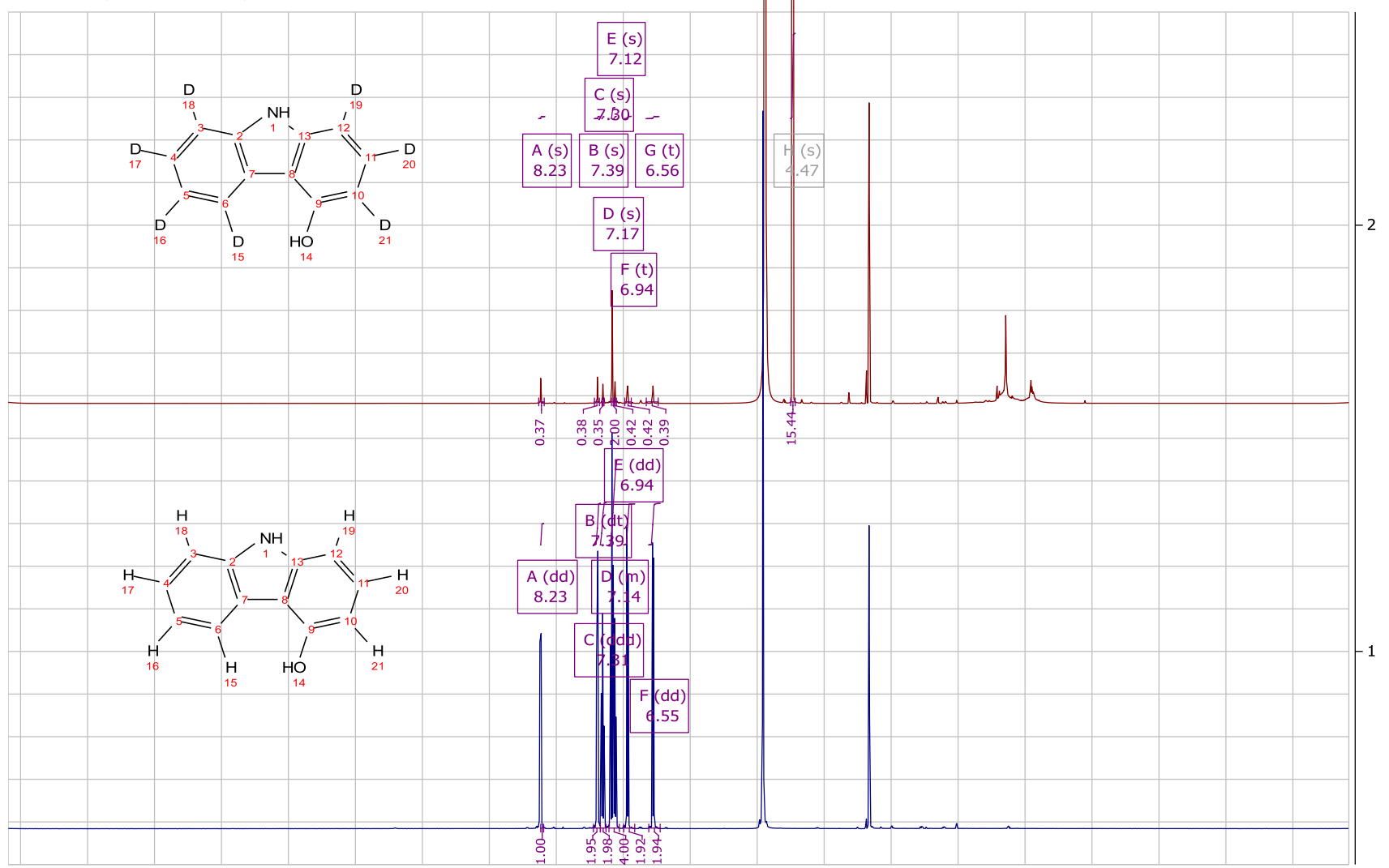

\begin{tabular}{lllllllllllllllllllll}
\hline 16 & 15 & 14 & 13 & 12 & 11 & 10 & 9 & 8 & 7 & 6 & 5 & 4 & 3 & 2 & 1 & 0 & -1 & -2 & -3
\end{tabular} 
33

1H NMR (400 MHz, MeOD)
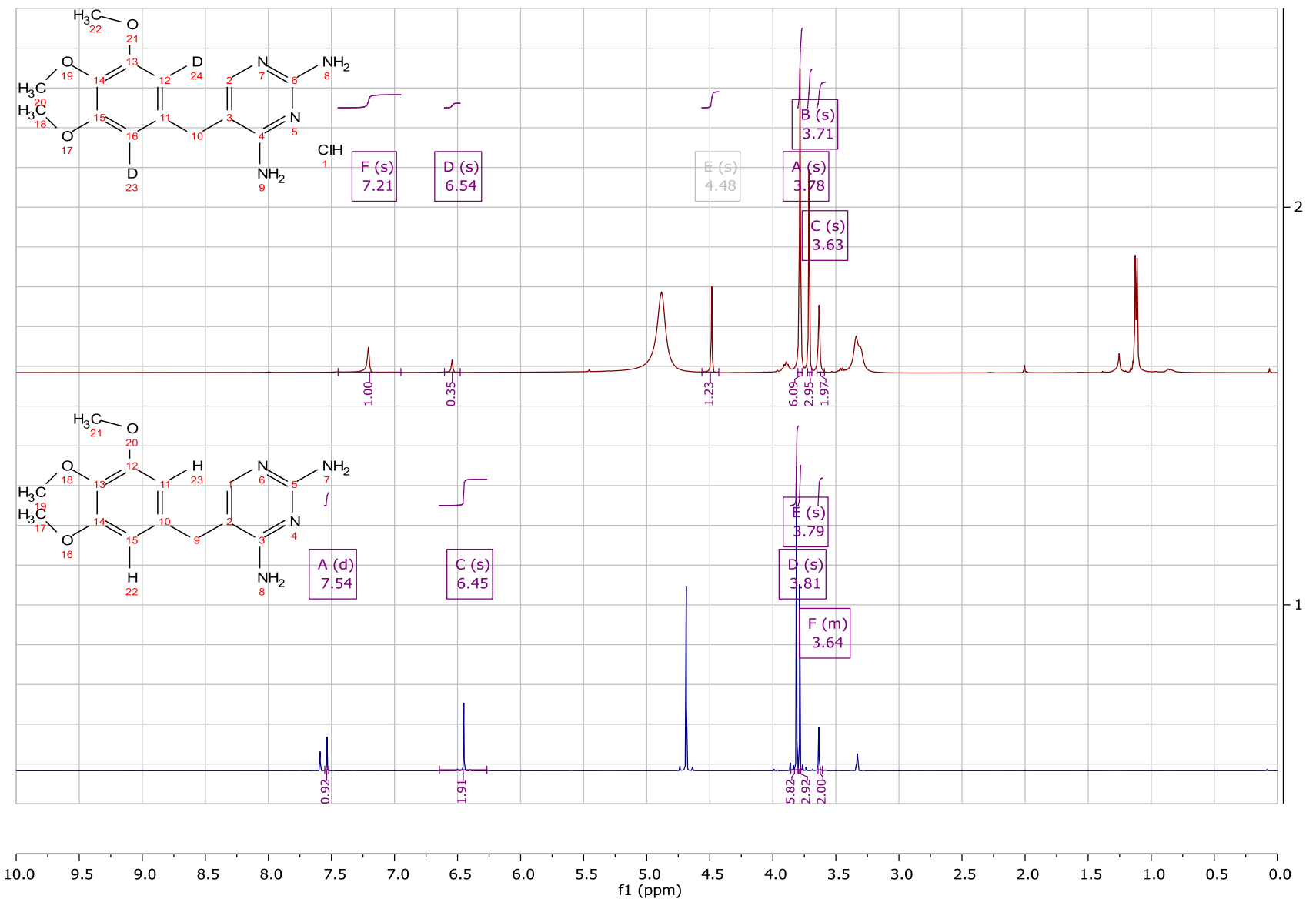


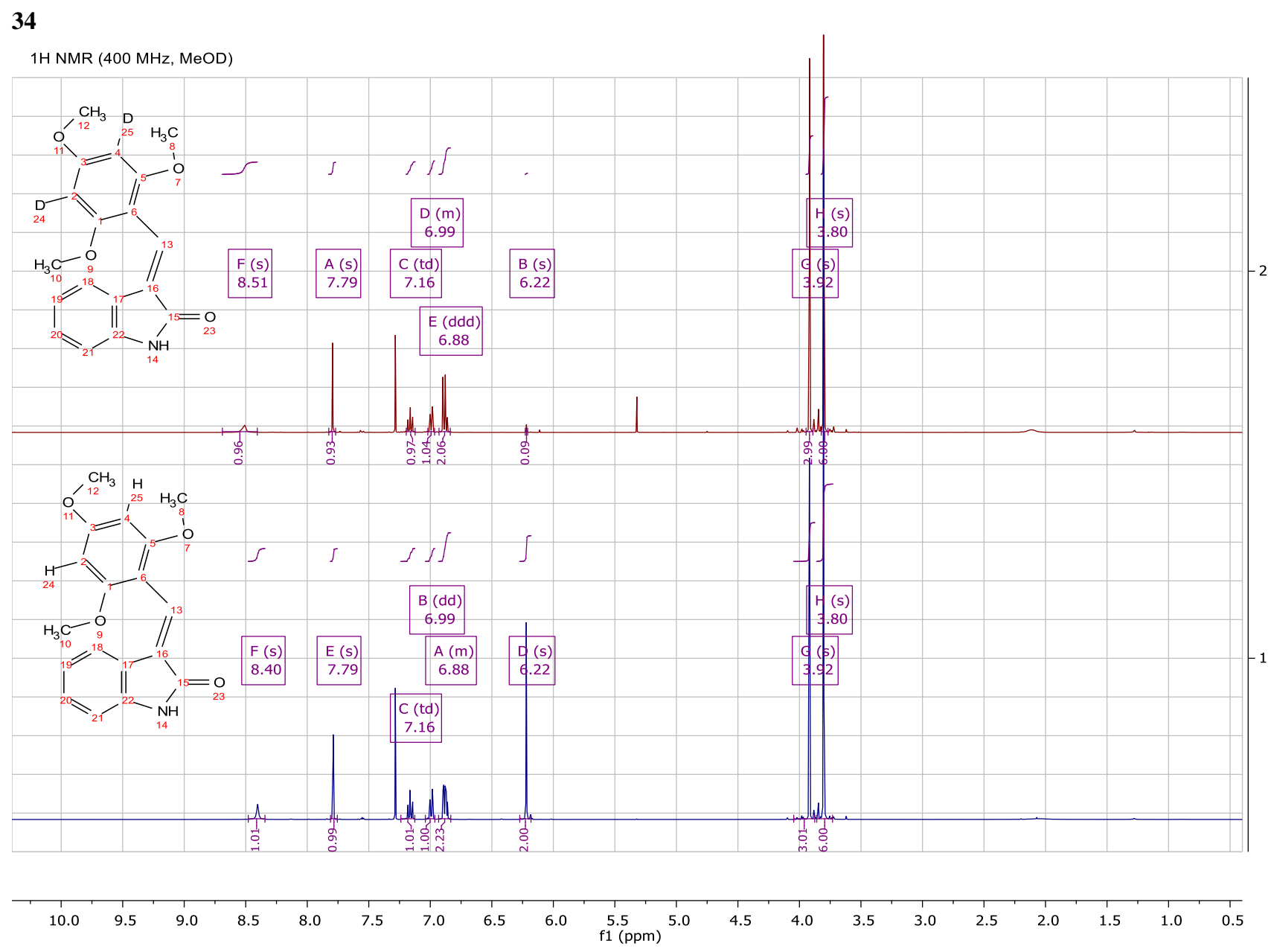


1H NMR (400 MHz, MeOD)

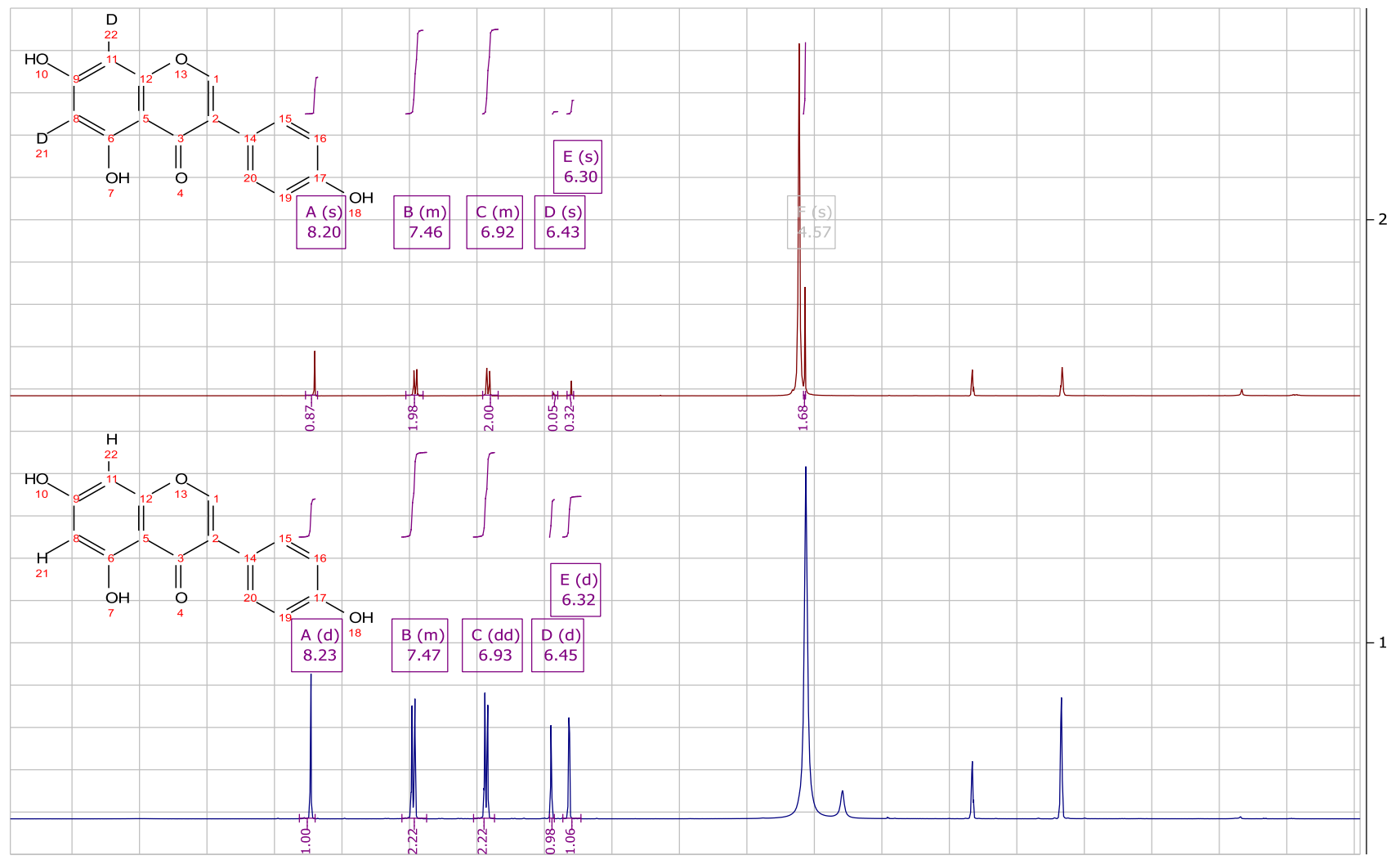

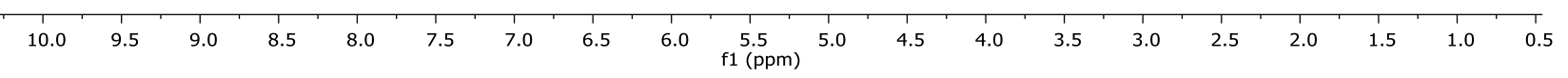


36

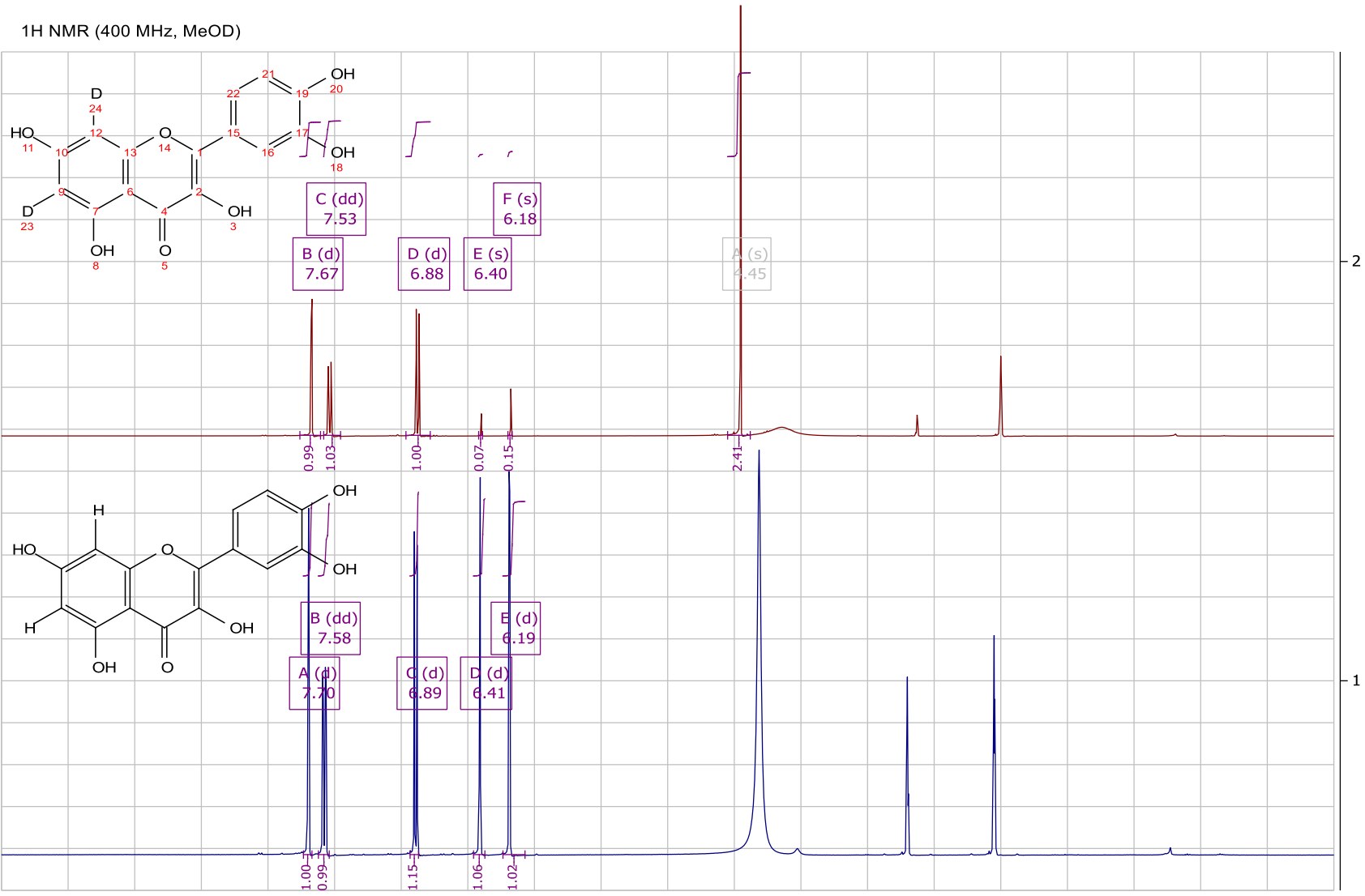

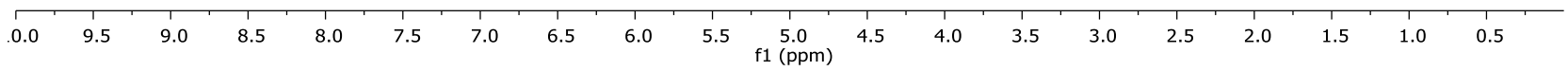


37

$1 \mathrm{H}$ NMR $(400 \mathrm{MHz}, \mathrm{MeOD})$

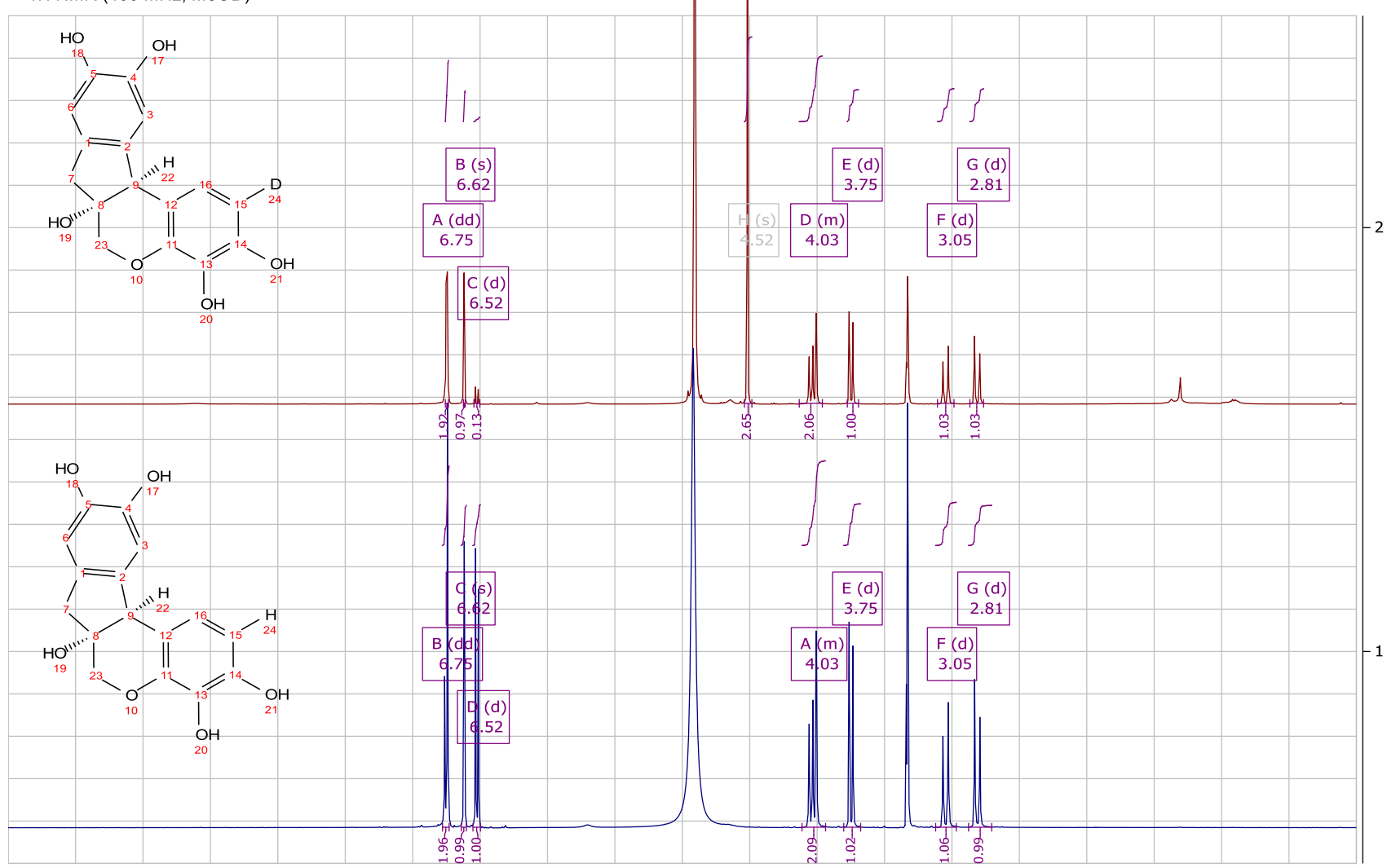

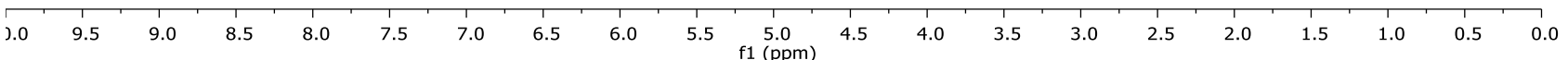



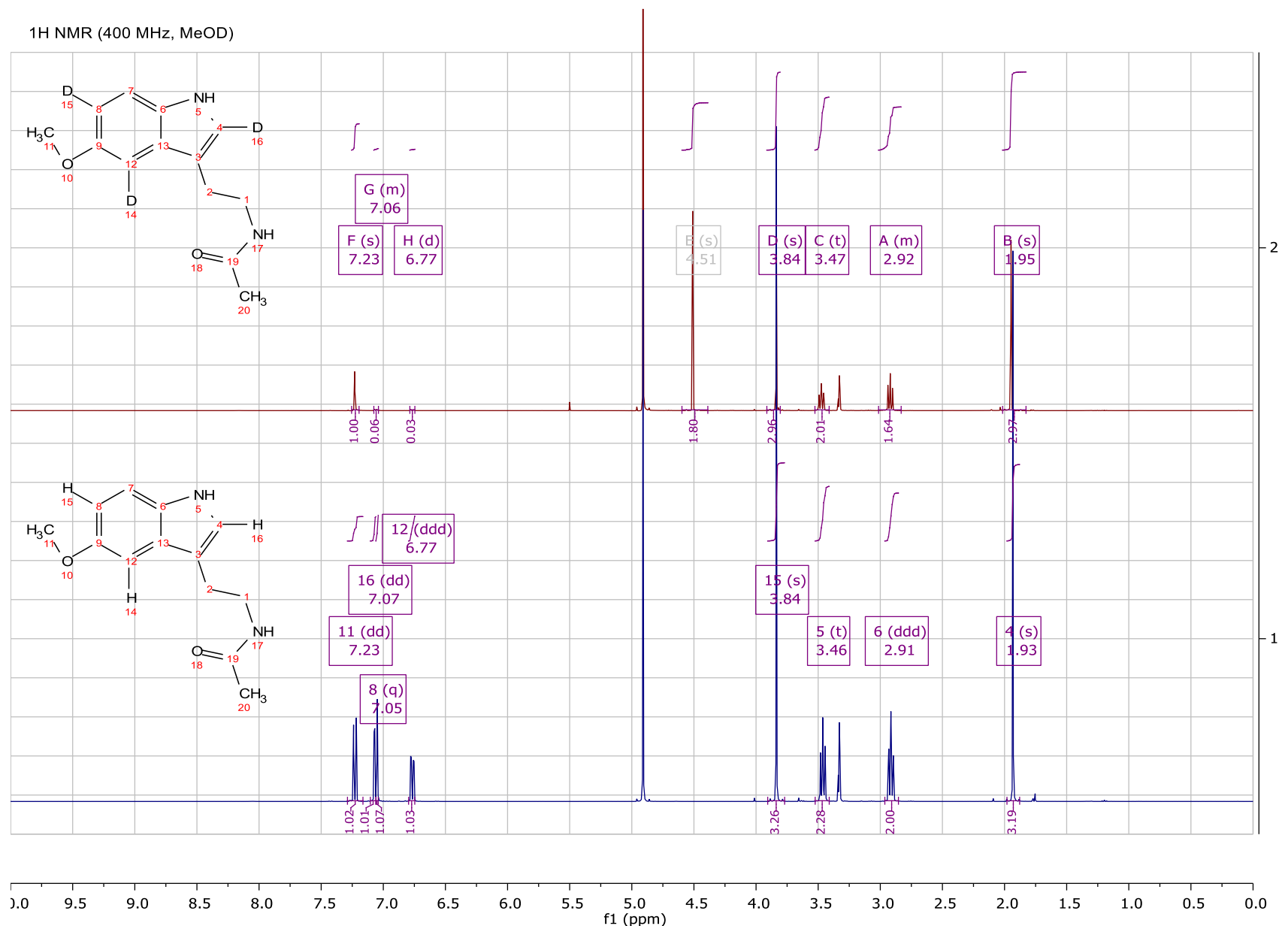

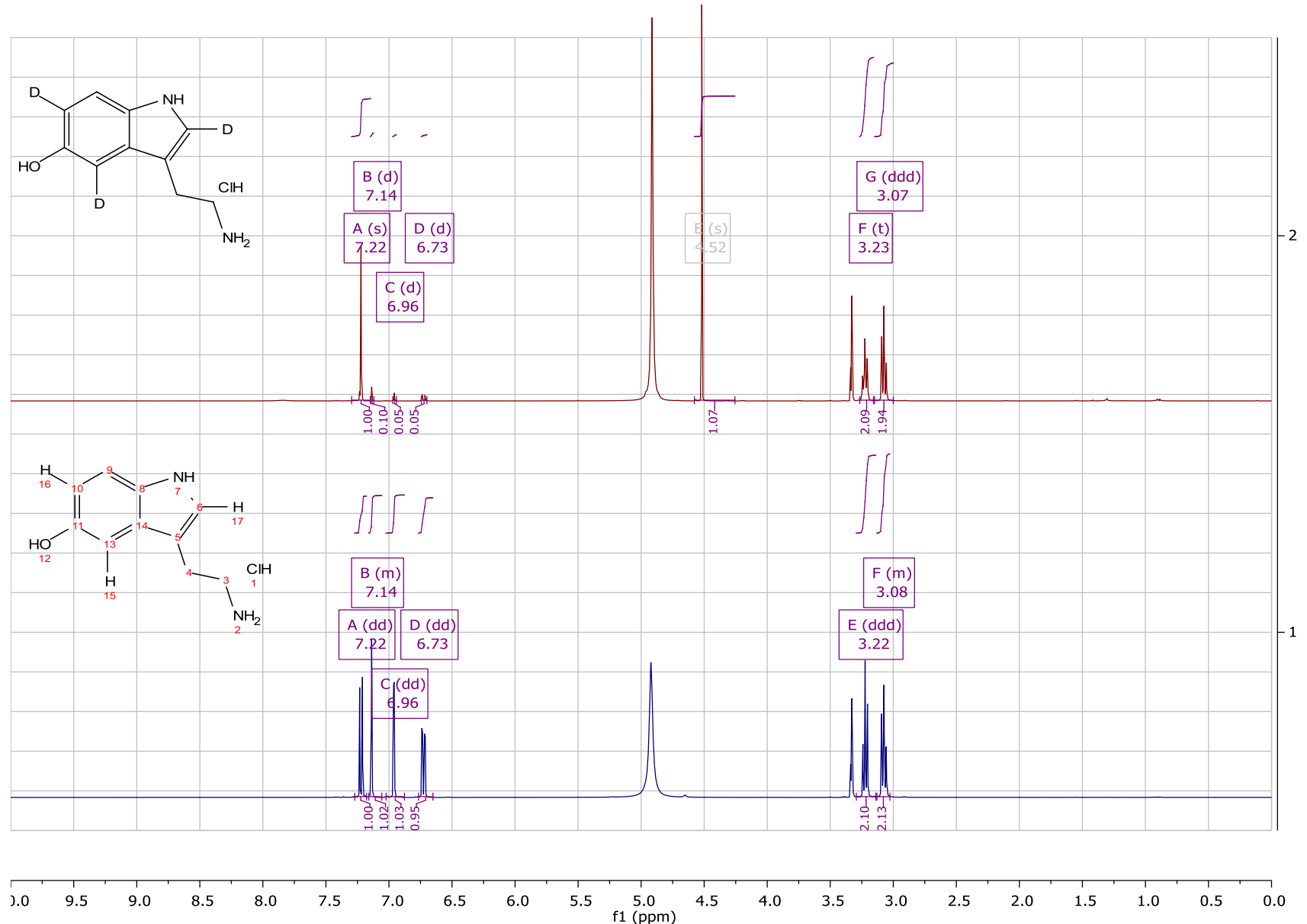

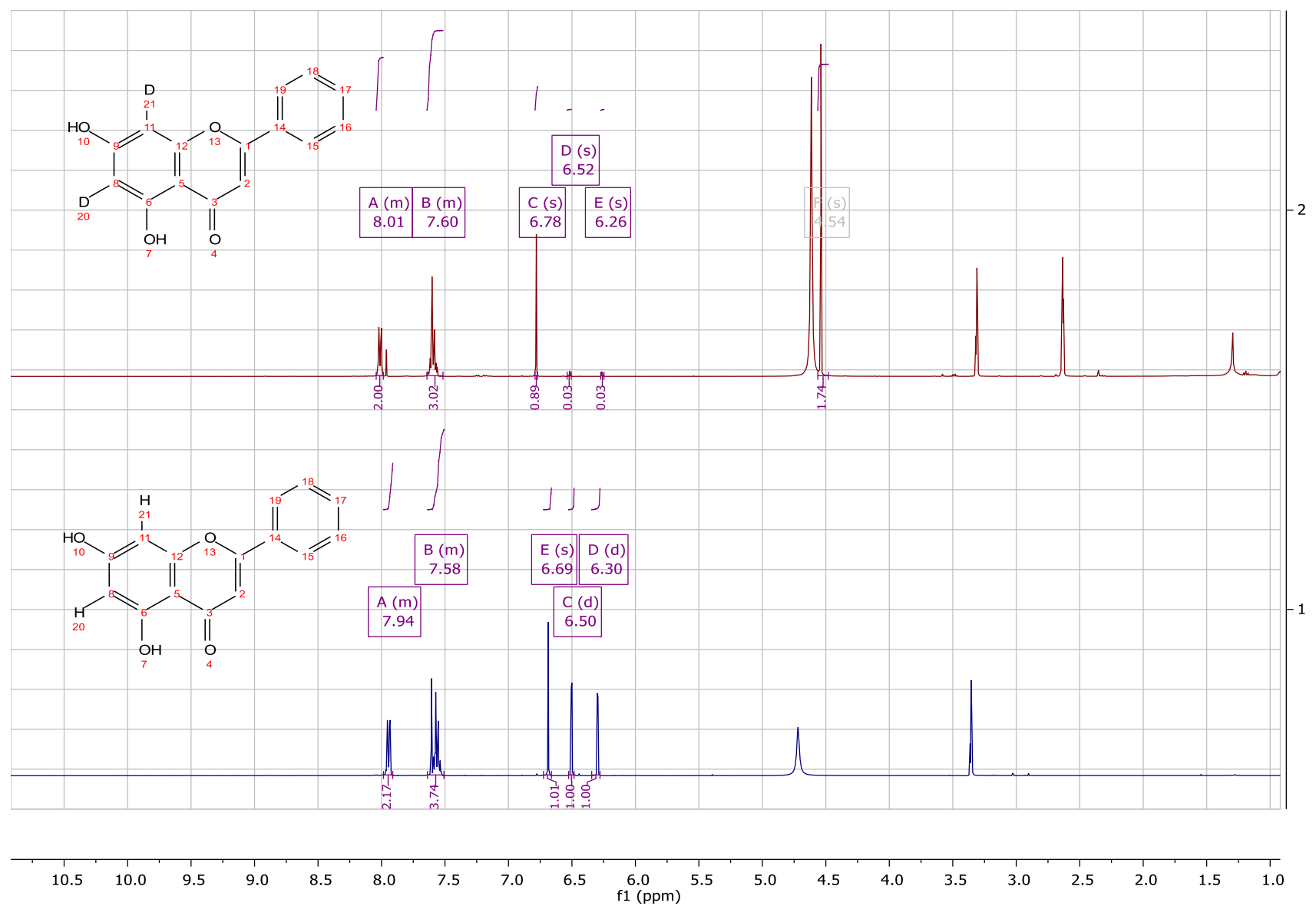
41

$1 \mathrm{H}$ NMR $(400 \mathrm{MHz}, \mathrm{MeOD})$

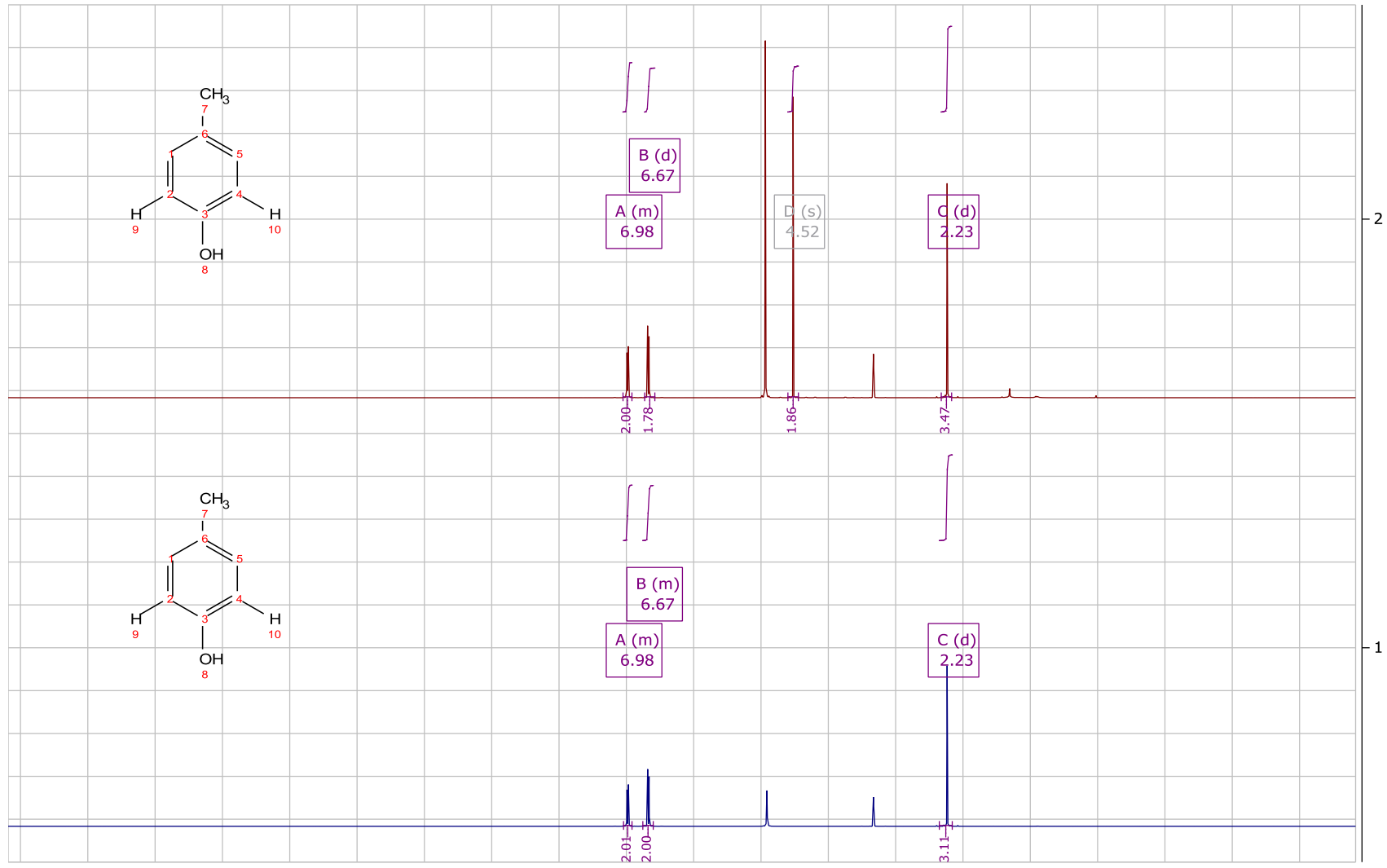

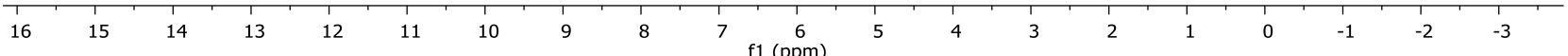


42

$1 \mathrm{H}$ NMR (400 MHz, MeOD)

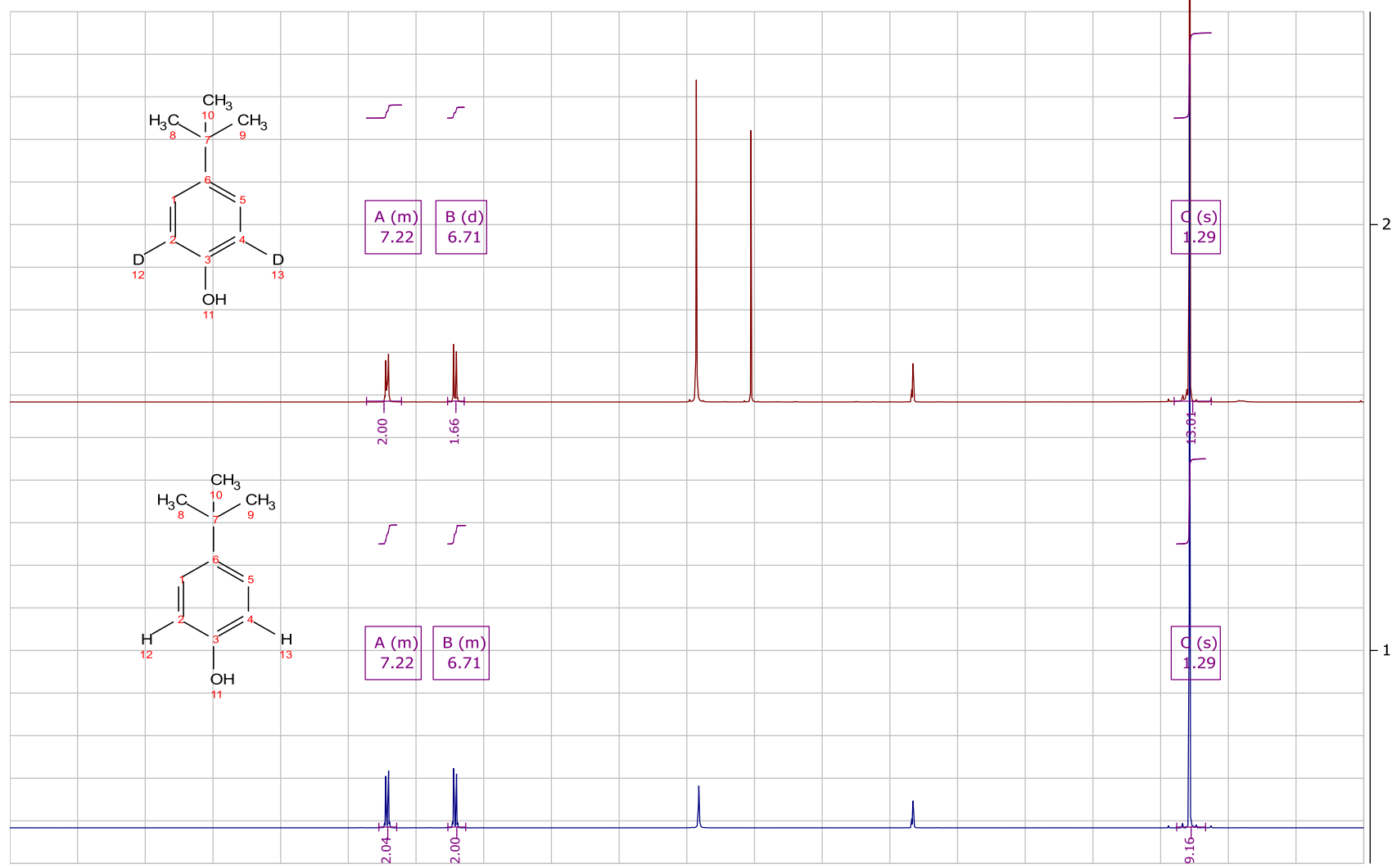

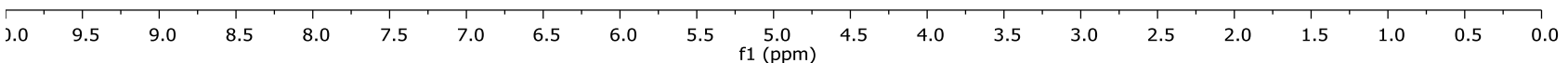



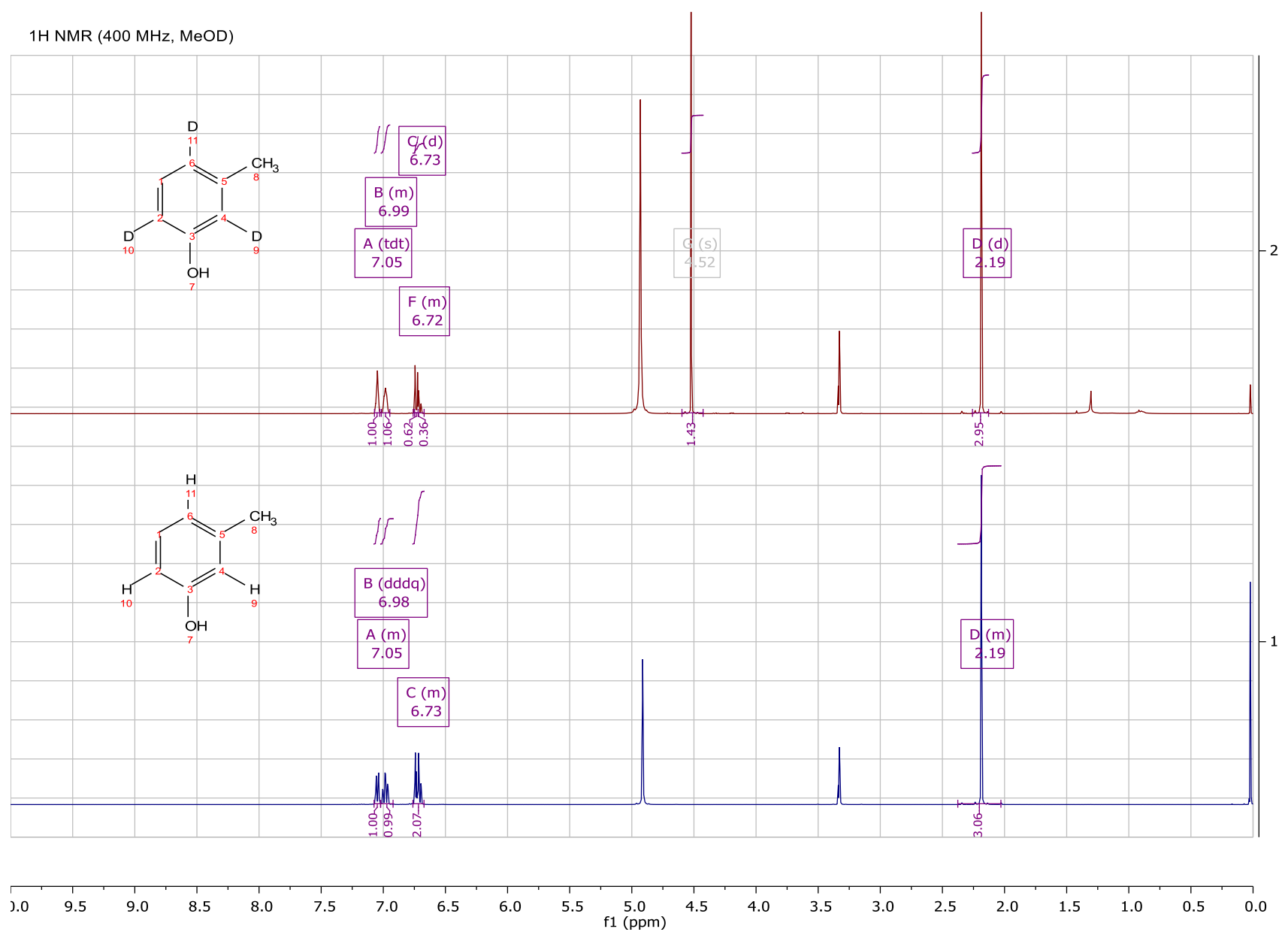
44

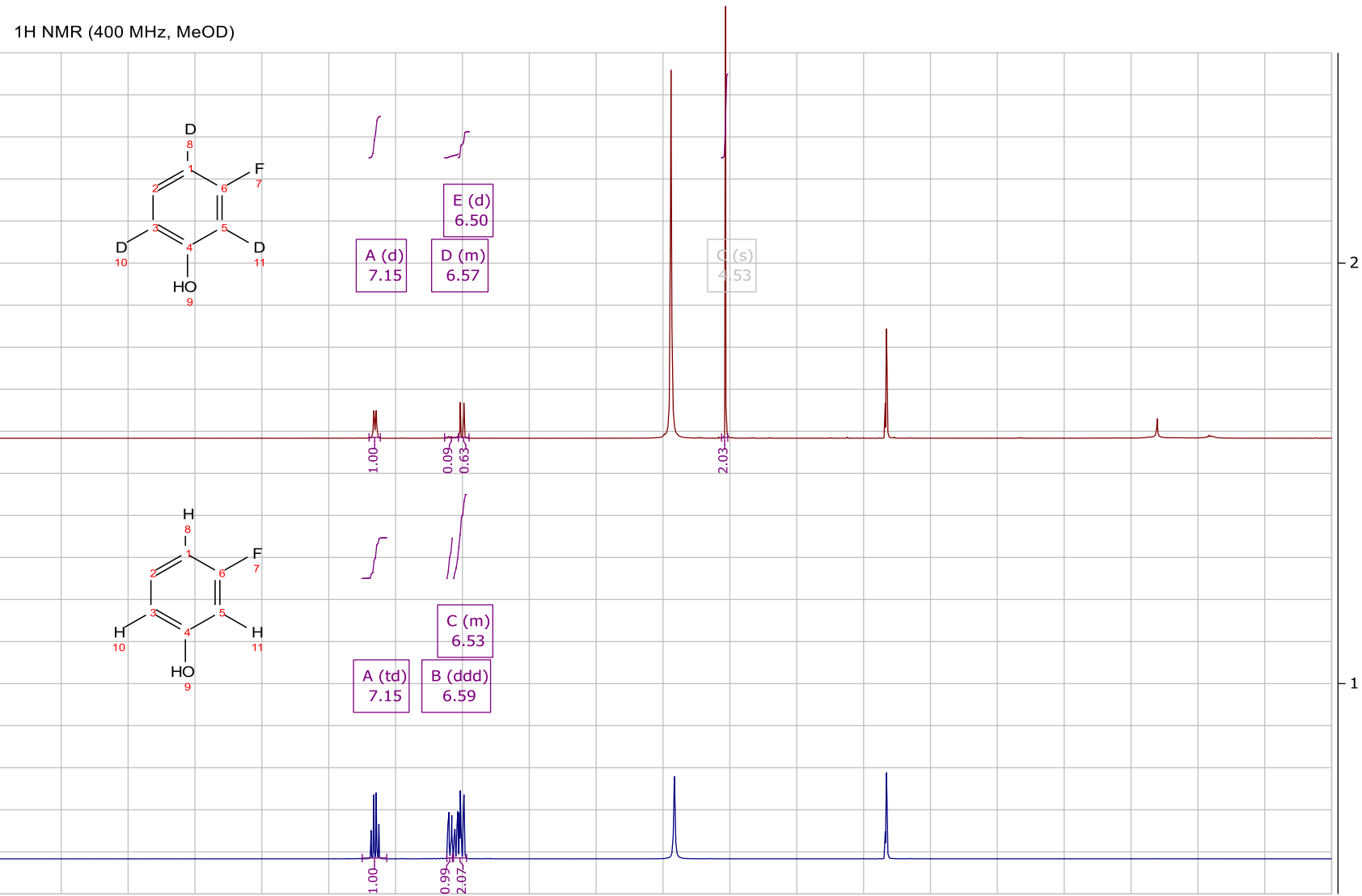

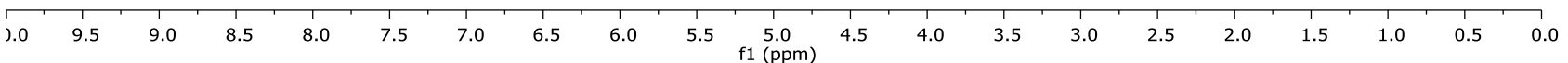



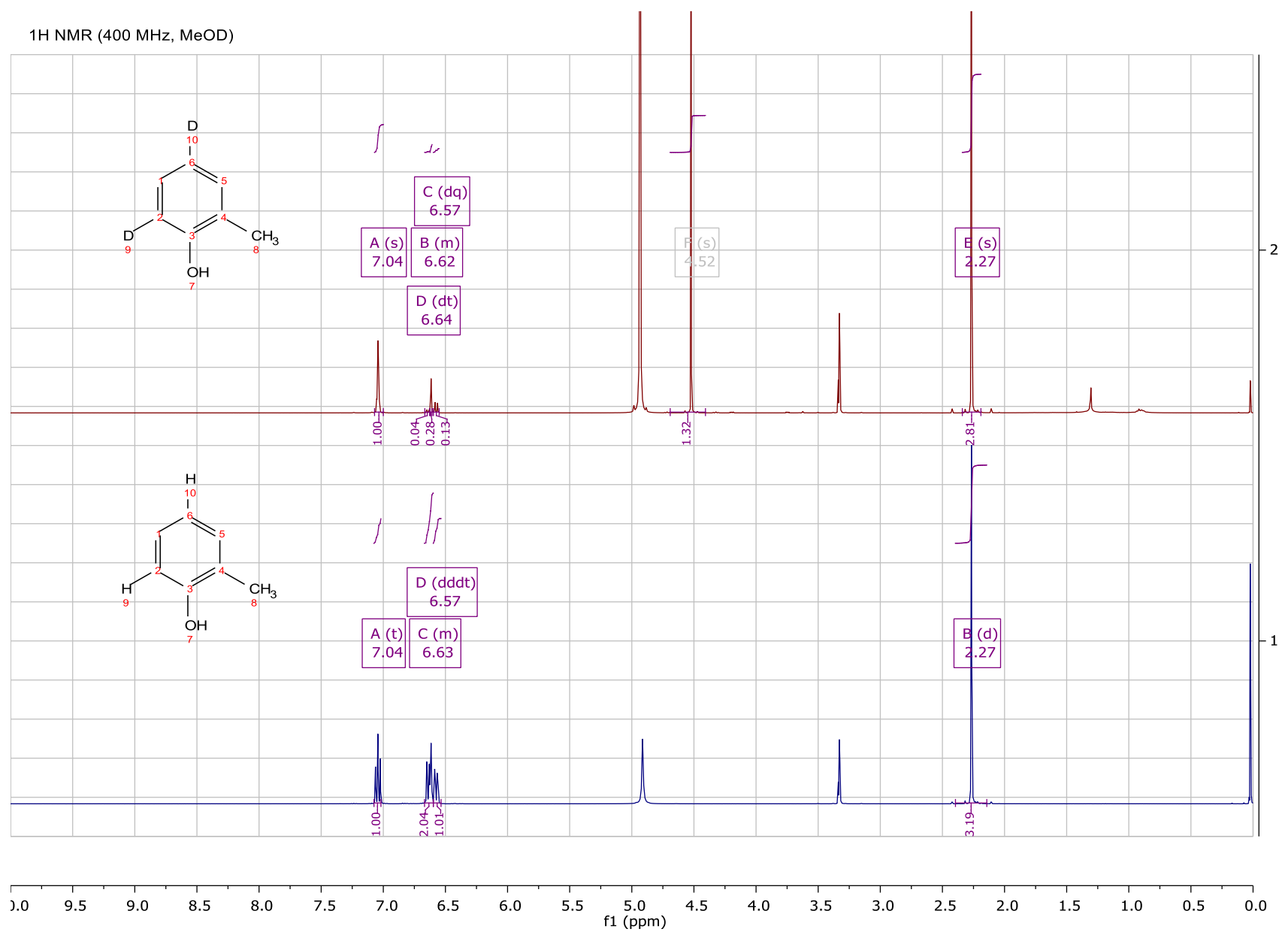
46

$1 \mathrm{H}$ NMR $(400 \mathrm{MHz}, \mathrm{MeOD})$

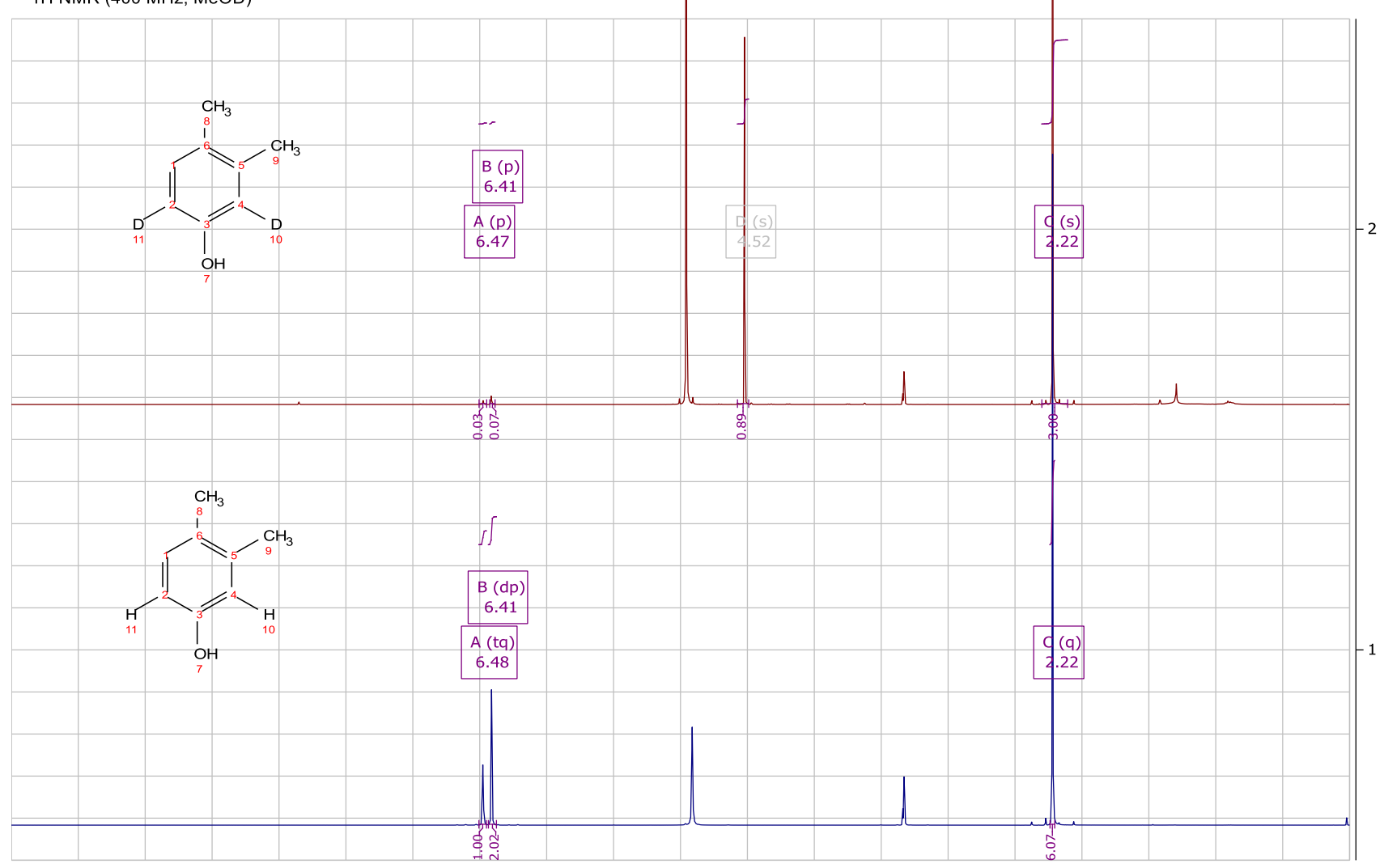

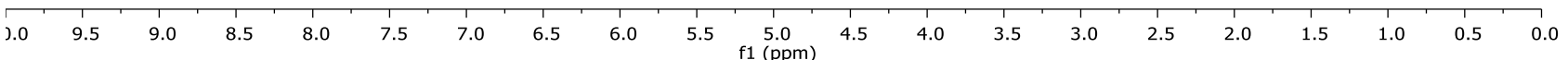


47

$1 \mathrm{H}$ NMR (400 MHz, MeOD)

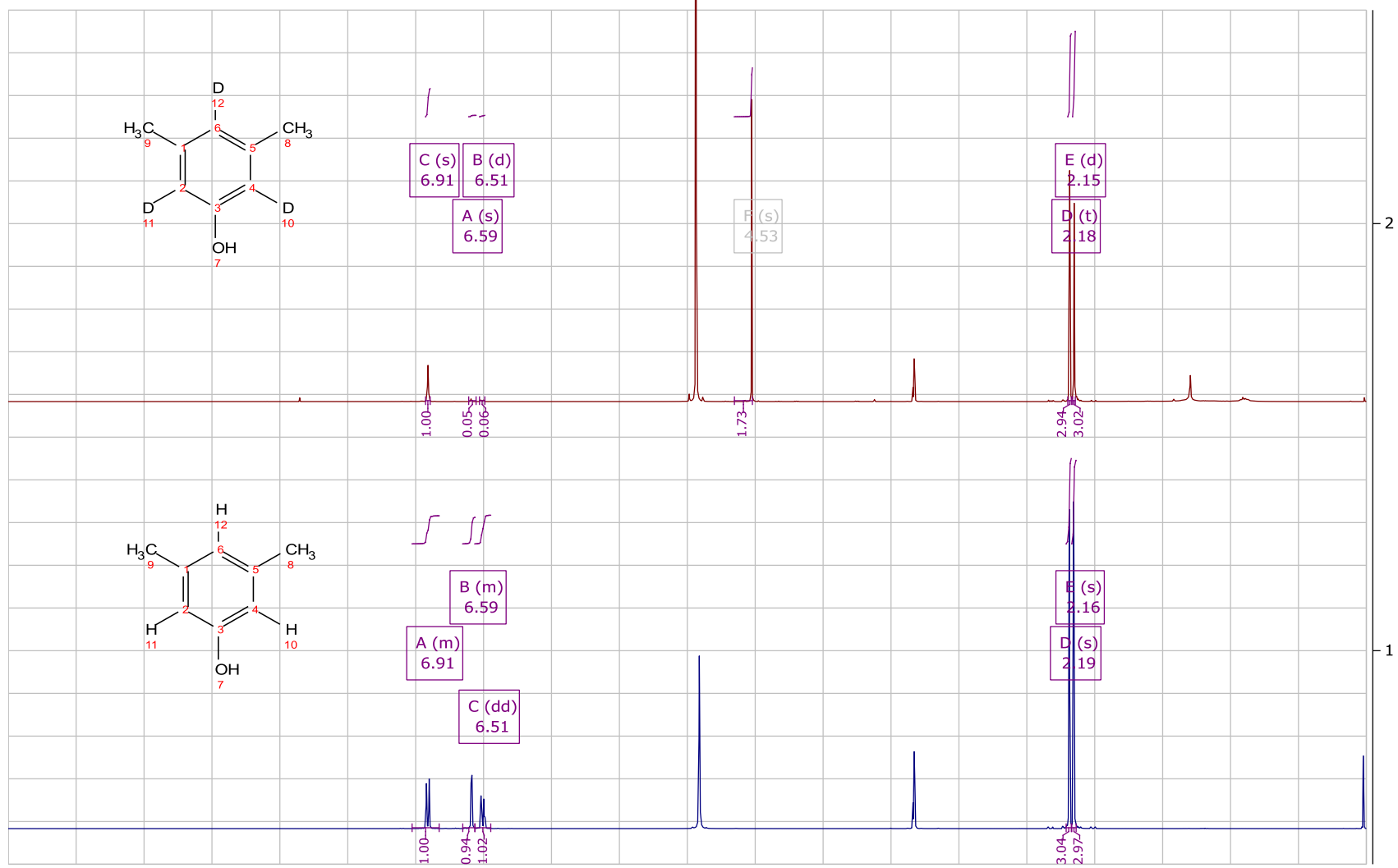

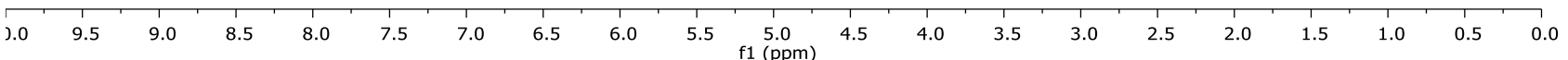


48

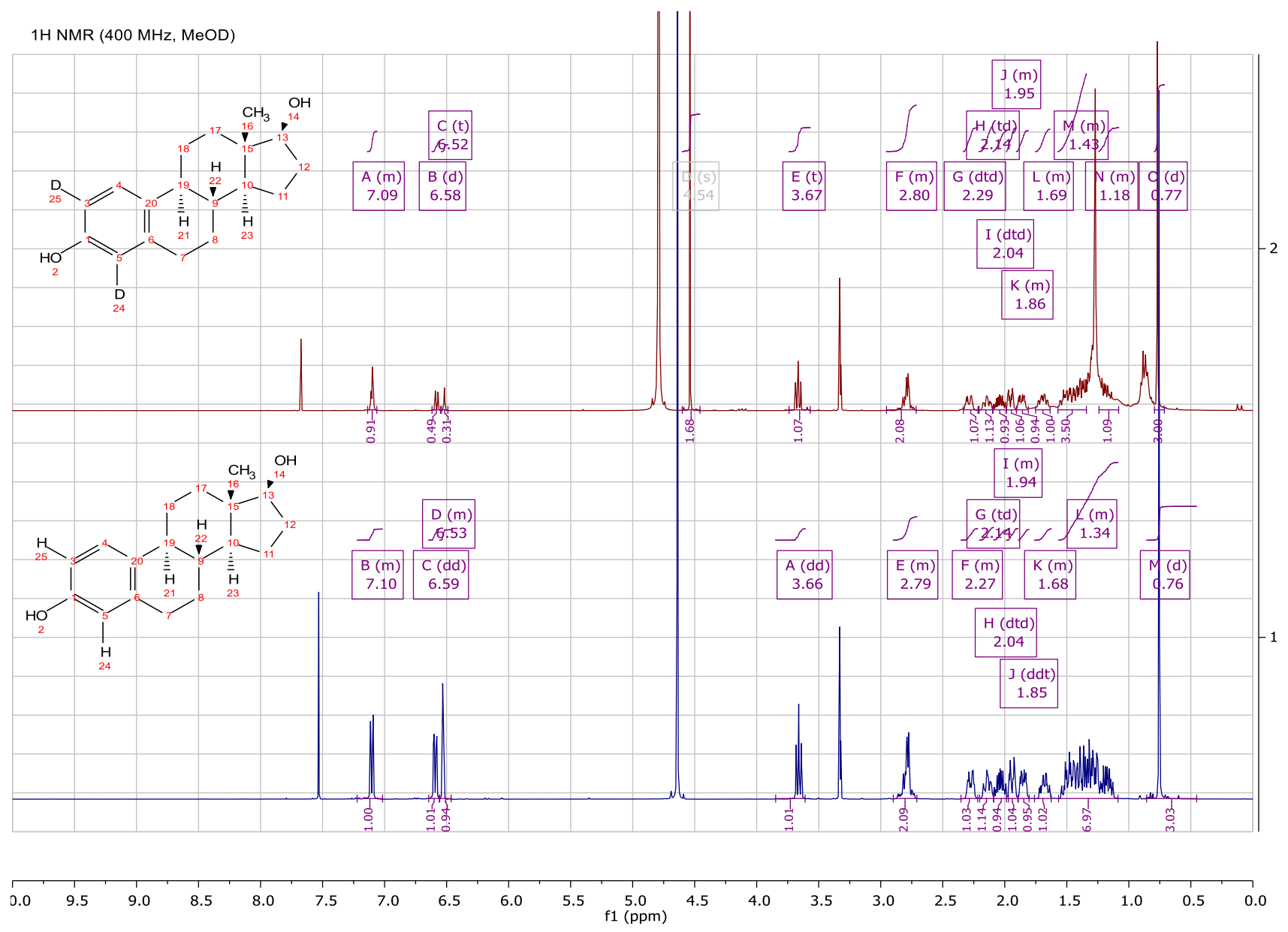



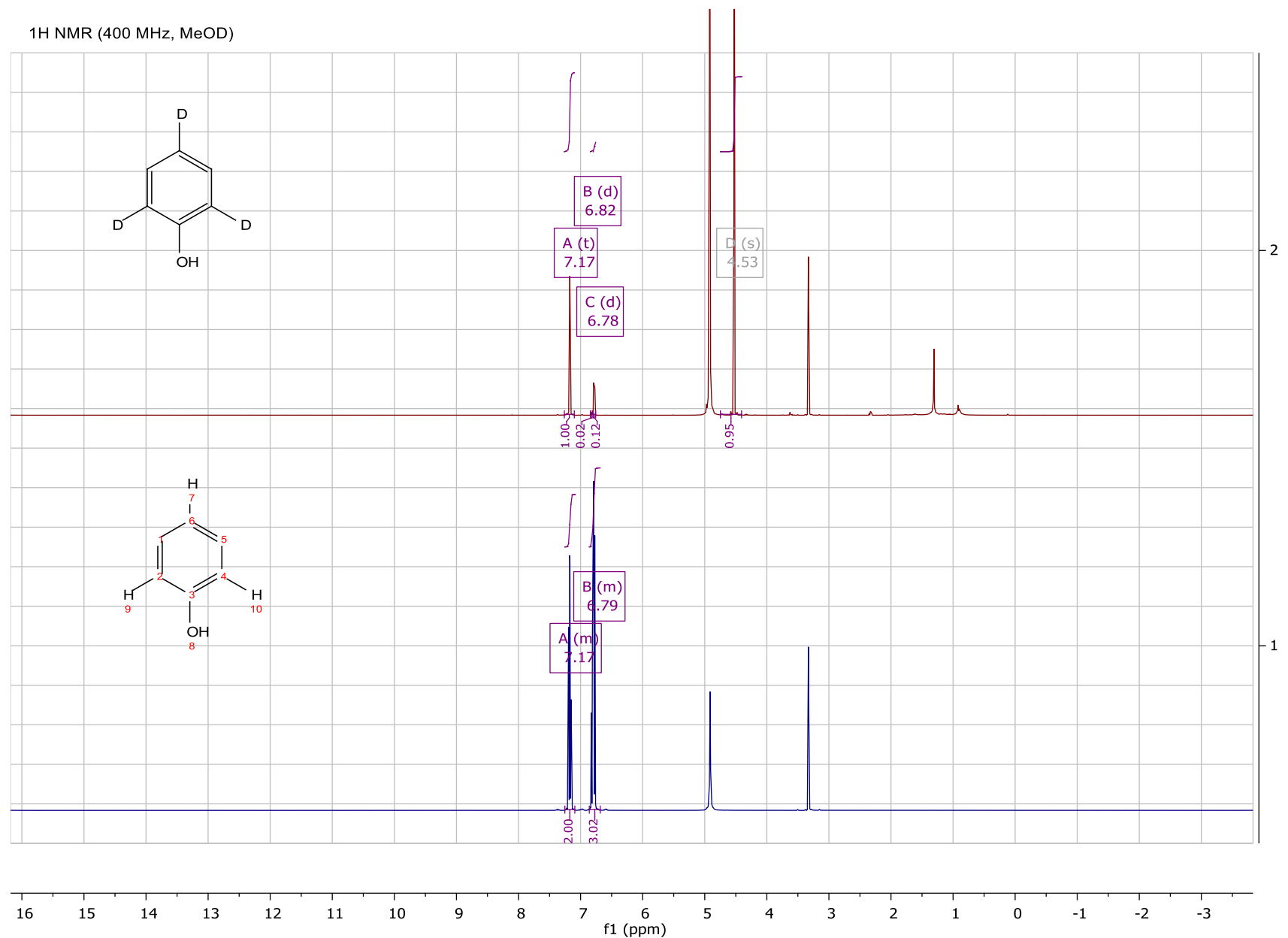


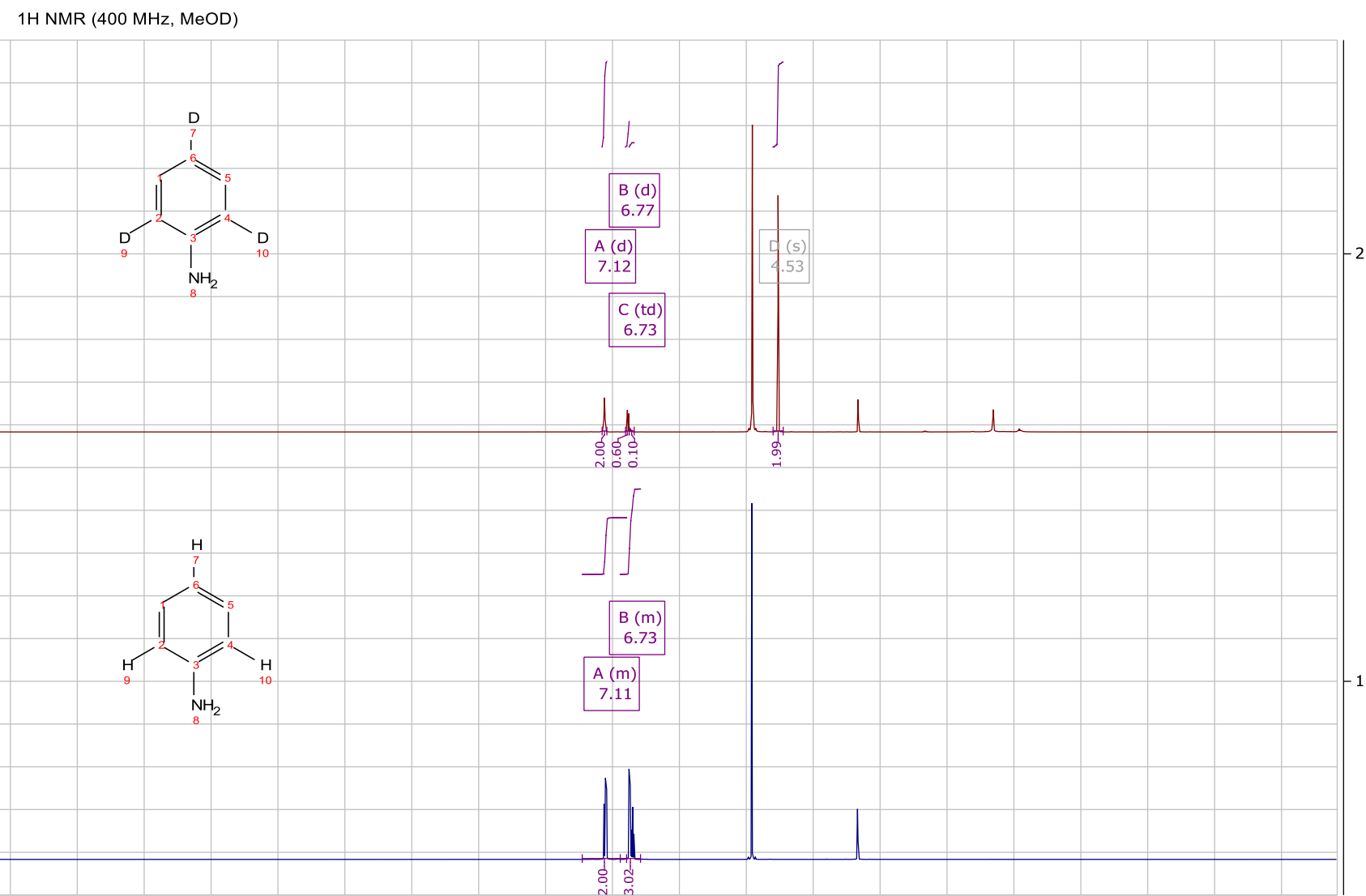


51
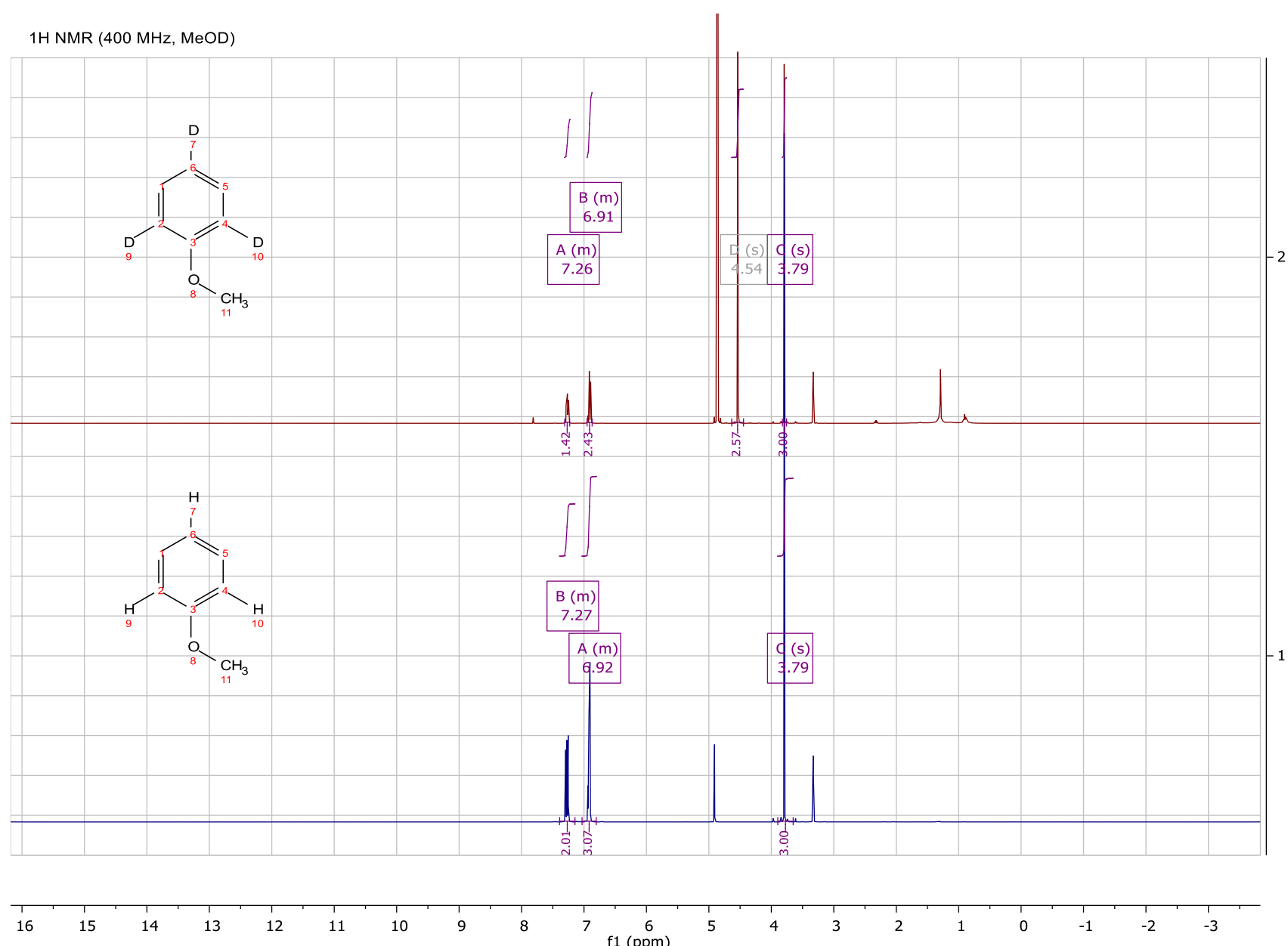
52

$1 \mathrm{H}$ NMR $(400 \mathrm{MHz}, \mathrm{MeOD})$

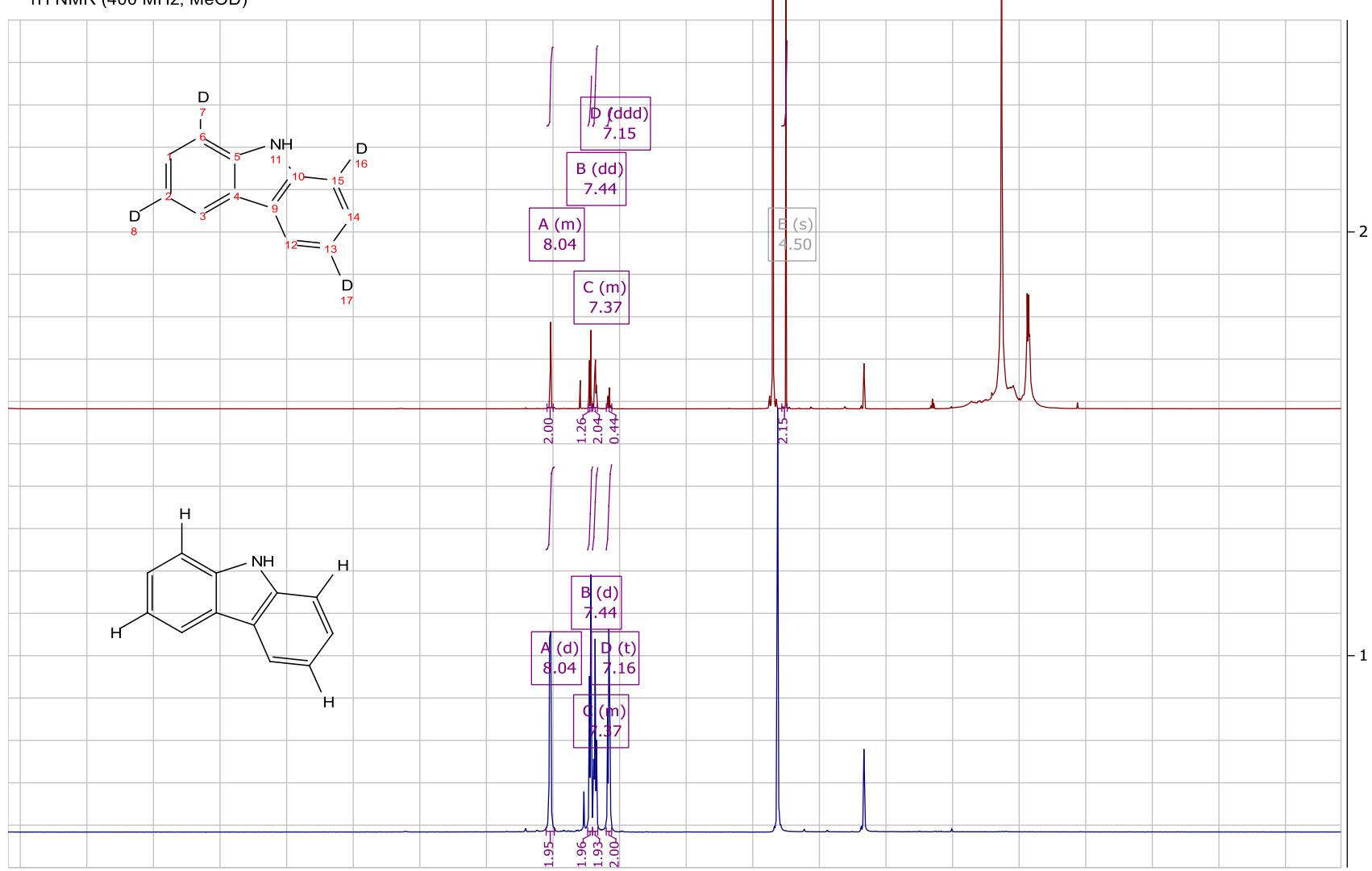

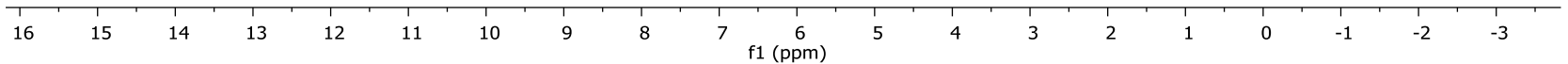




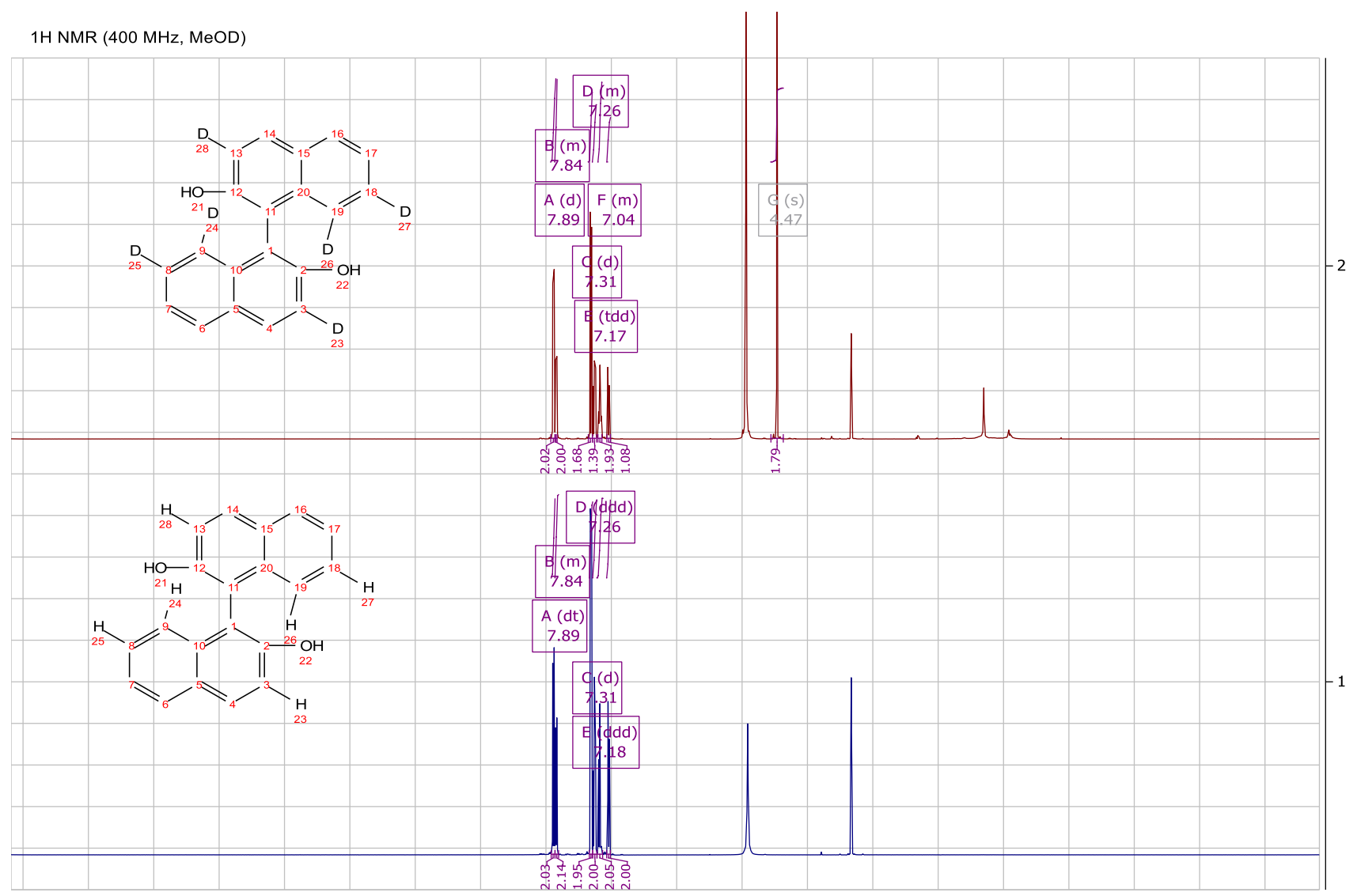

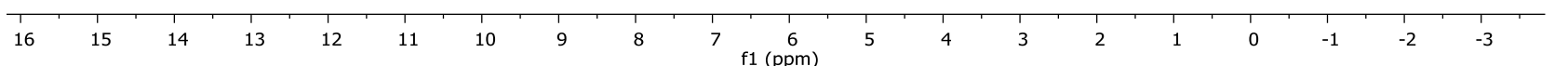


54

$1 \mathrm{H}$ NMR $(400 \mathrm{MHz}, \mathrm{MeOD})$

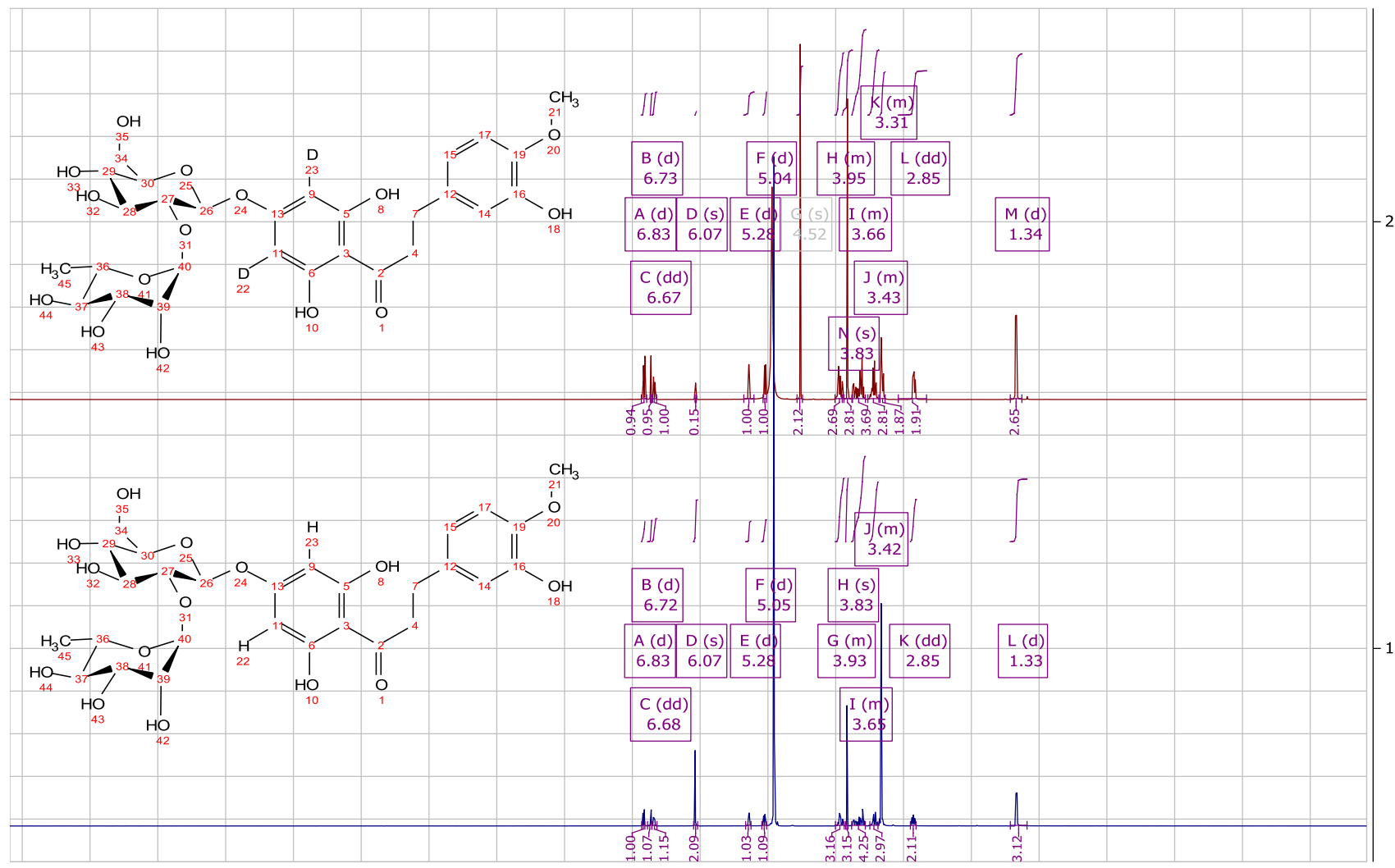

\begin{tabular}{|c|c|c|c|c|c|c|c|c|c|c|c|c|c|c|c|c|c|c|c|}
\hline 16 & 15 & 14 & 13 & 12 & 11 & 10 & 9 & 8 & 7 & $\begin{array}{c}6 \\
\mathrm{f} 1(\mathrm{ppm})\end{array}$ & 5 & 4 & 3 & 2 & 1 & 0 & -1 & -2 & -3 \\
\hline
\end{tabular}



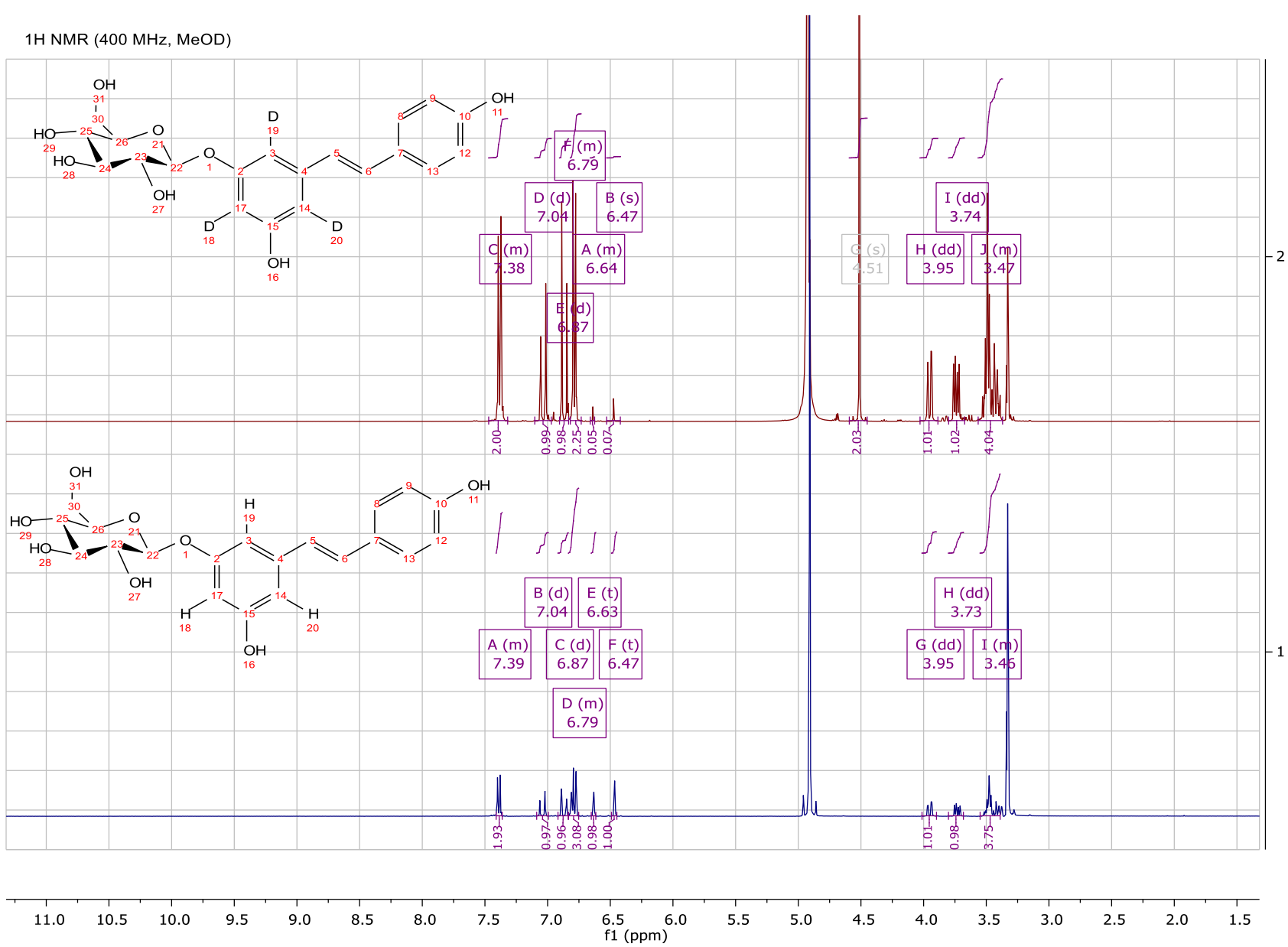


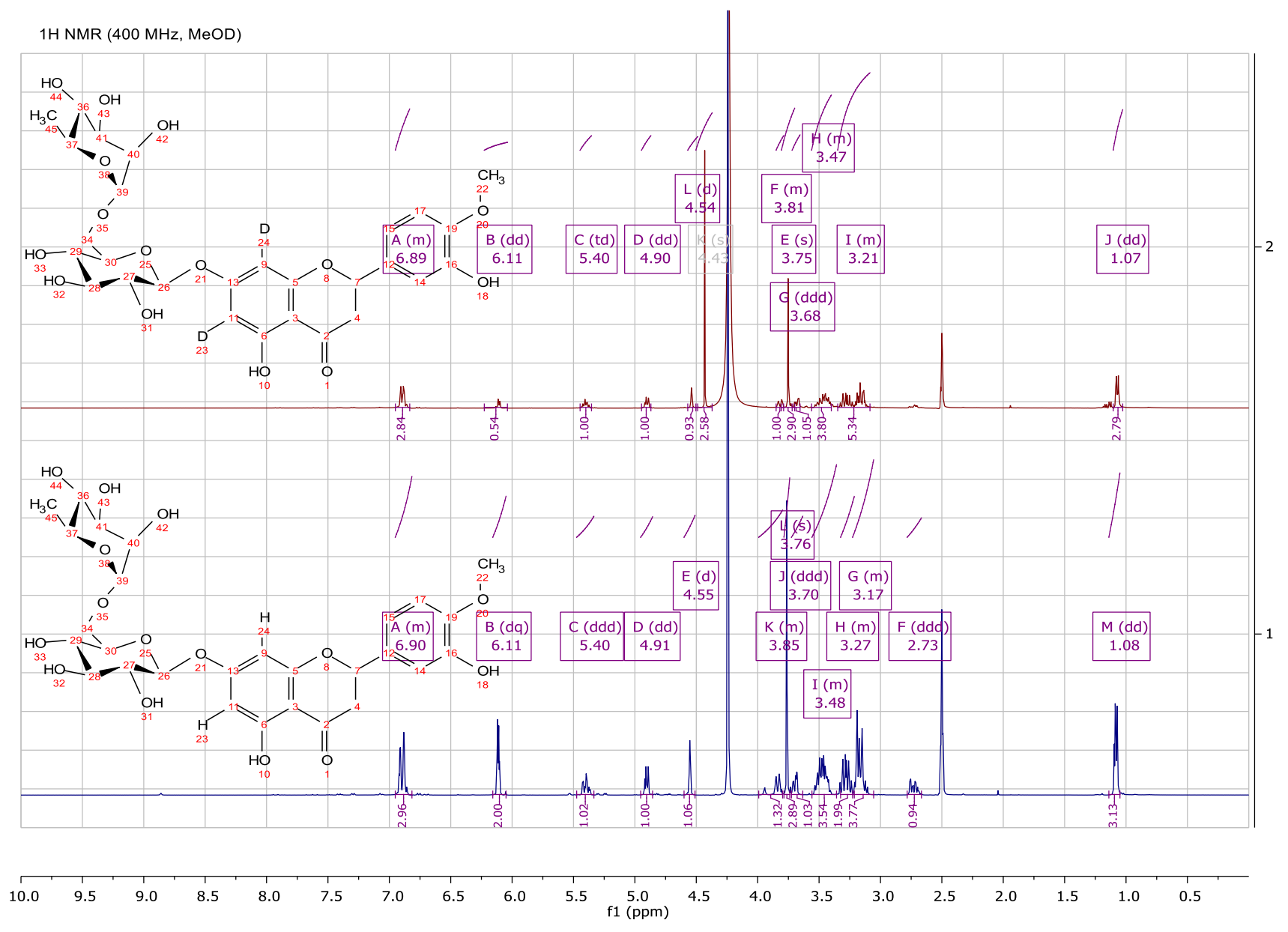


57a (5 h at rt)
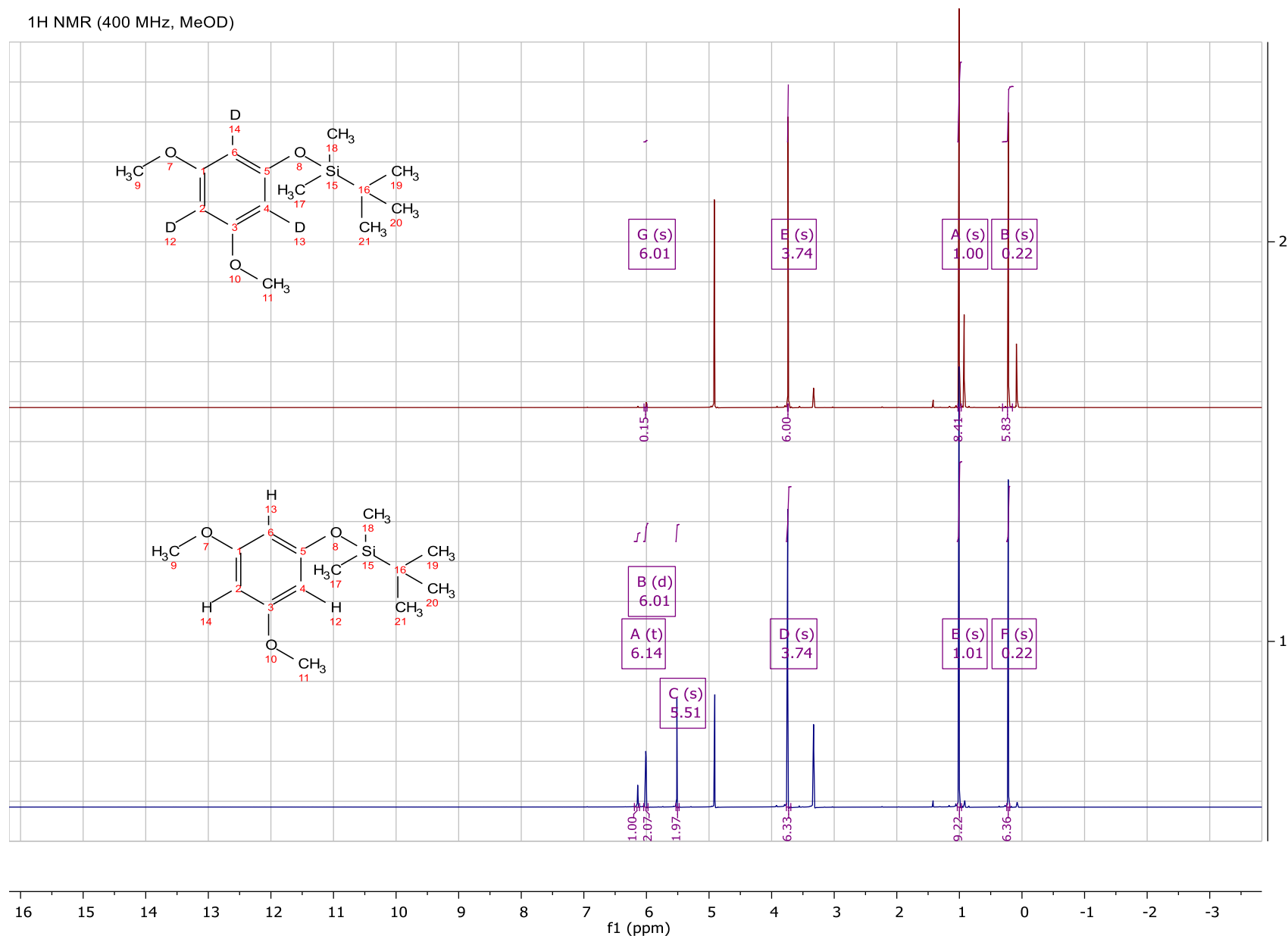
$57 b(5$ h at rt)

1H NMR (400 MHz, MeOD)

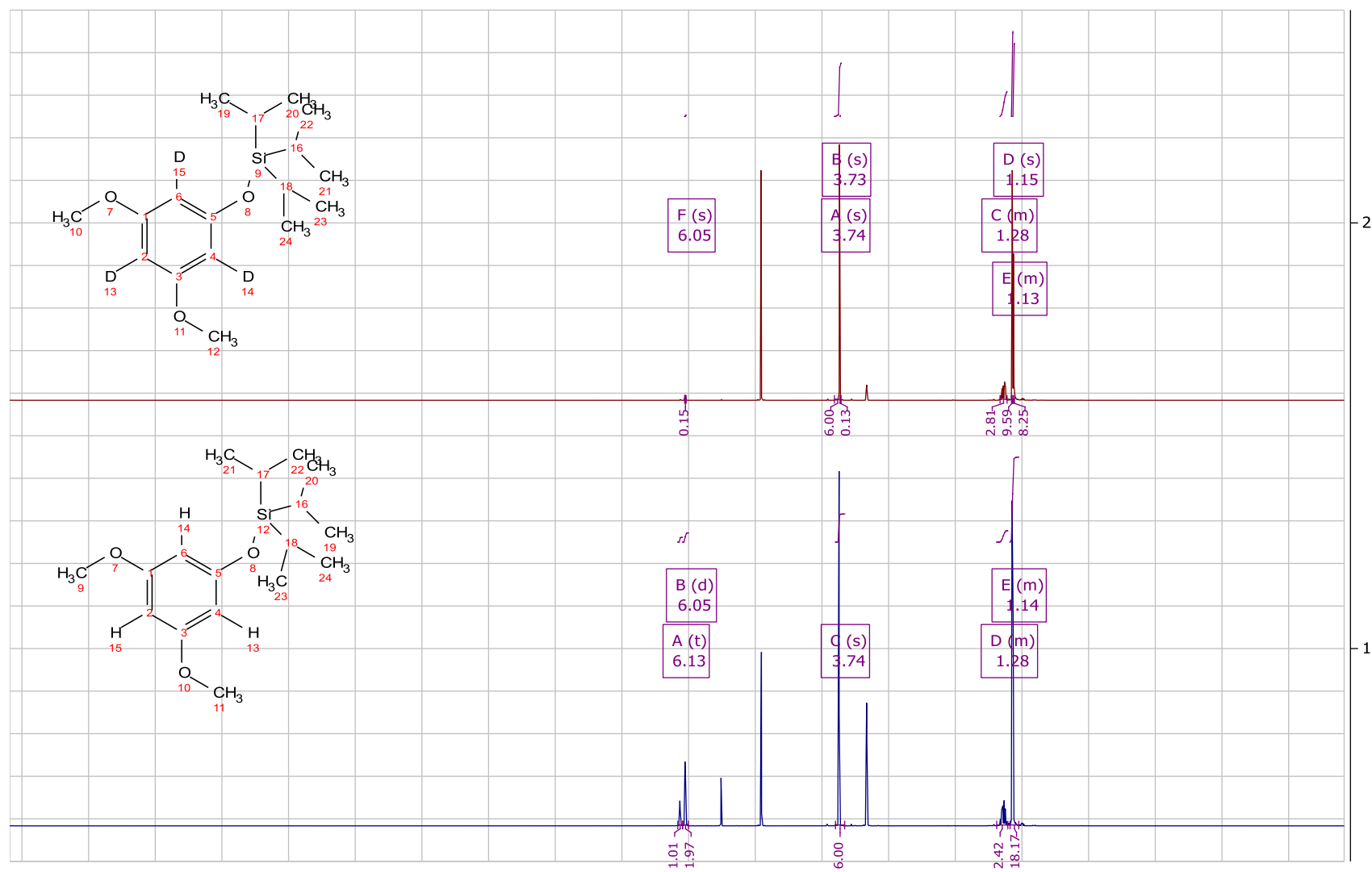

16 


\section{$57 \mathrm{c}(5 \mathrm{~h}$ at $\mathrm{rt})$}
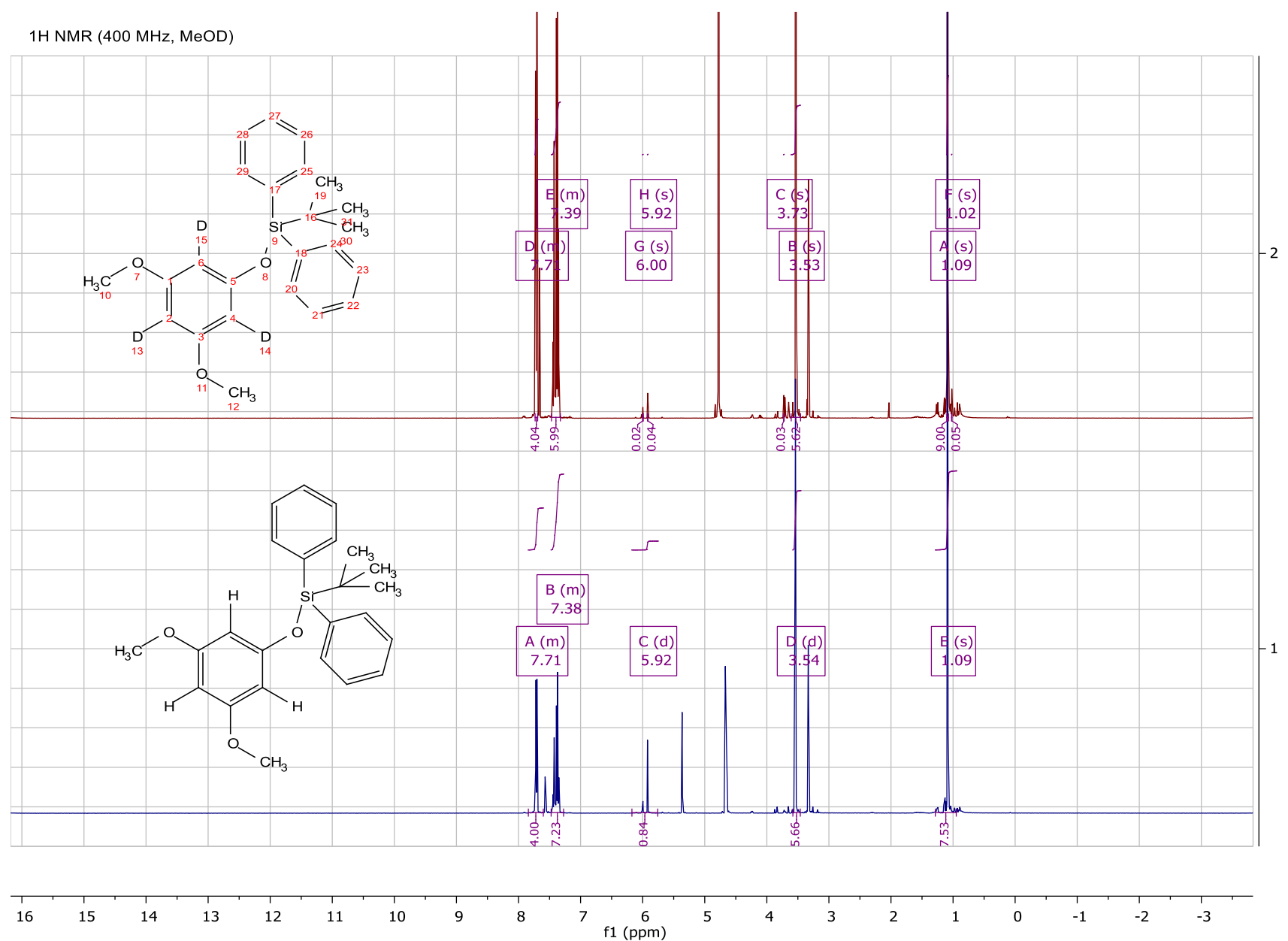


\section{Large scale reactions}

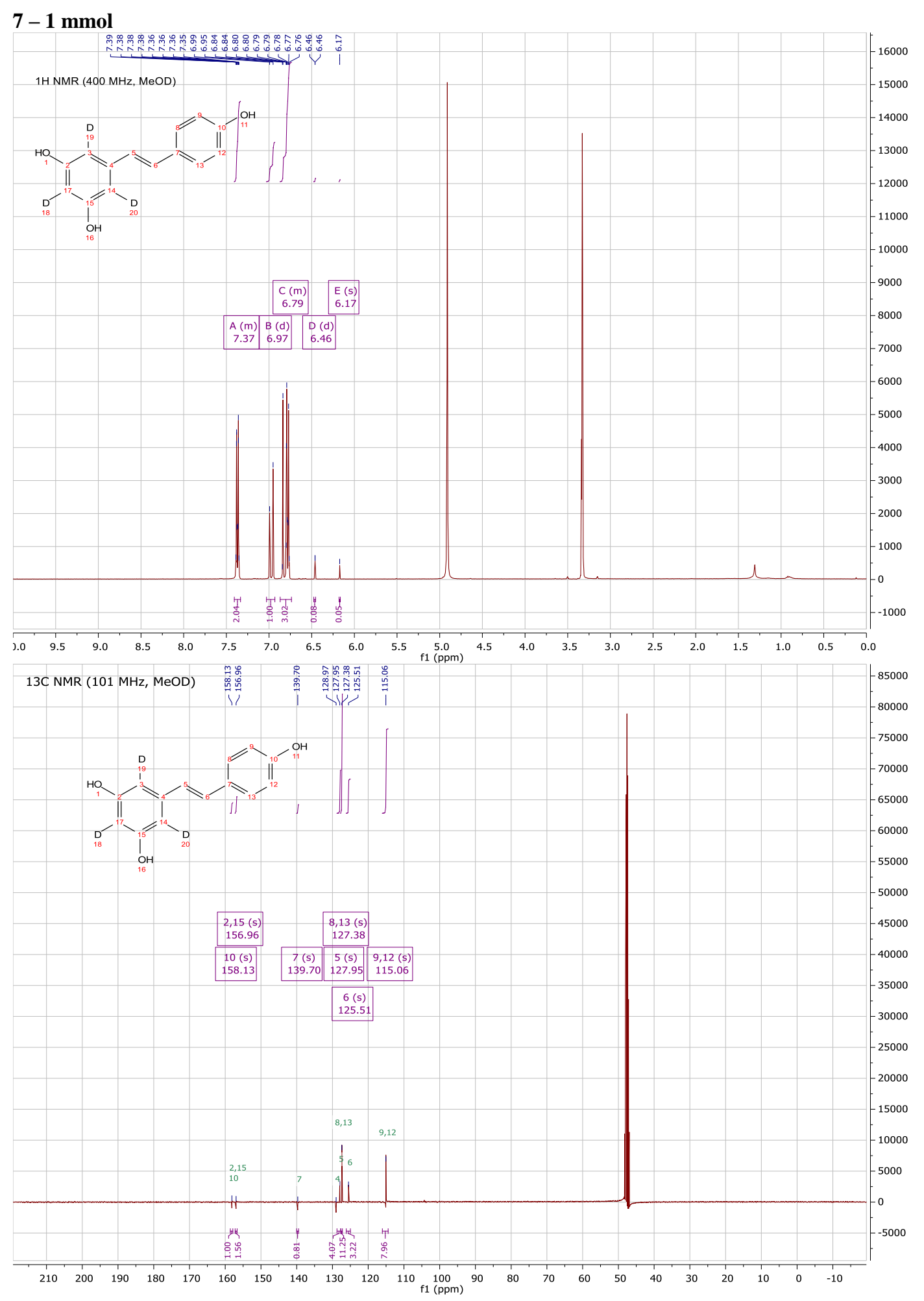



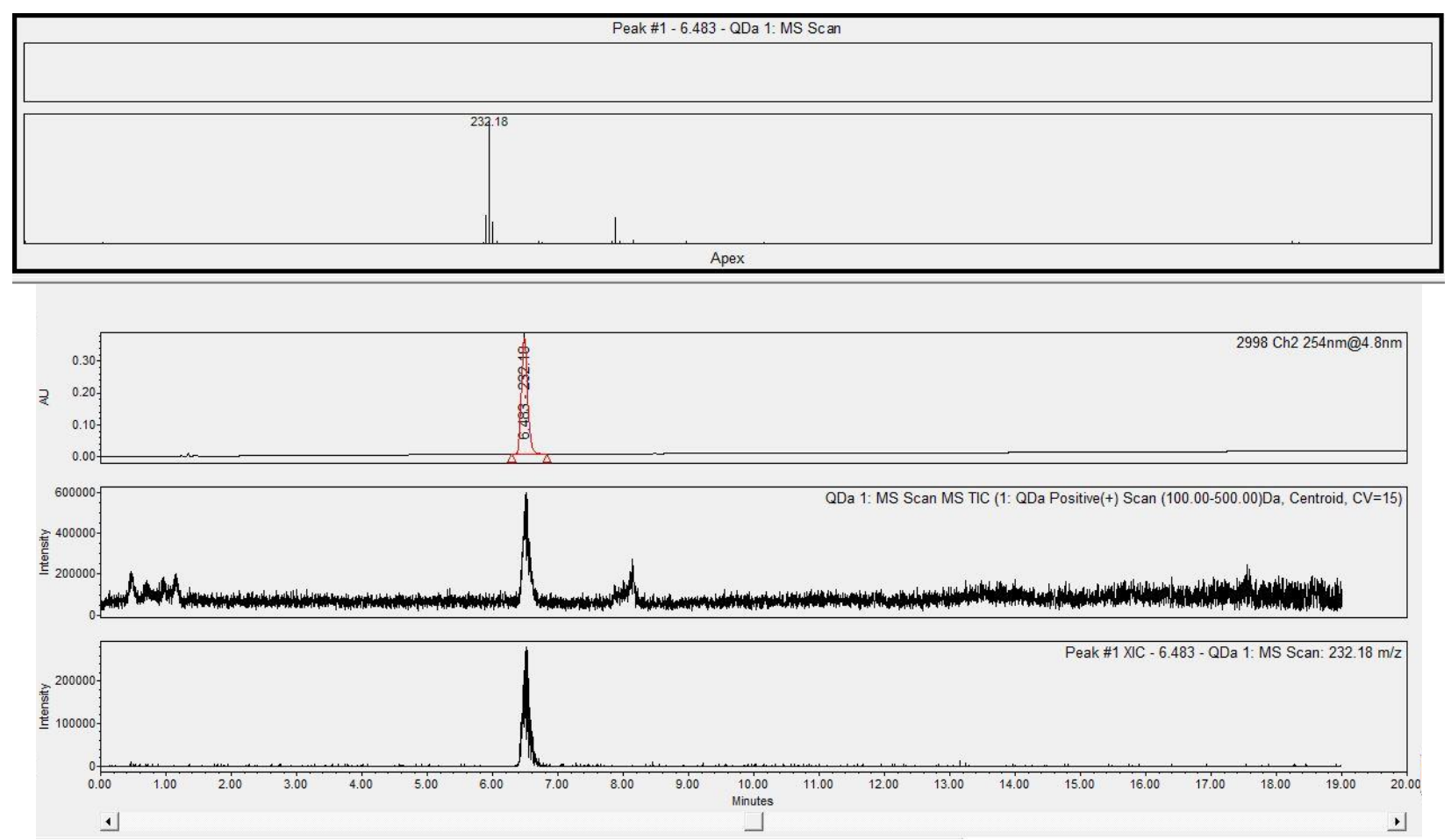


\section{4 - 1 mmol}

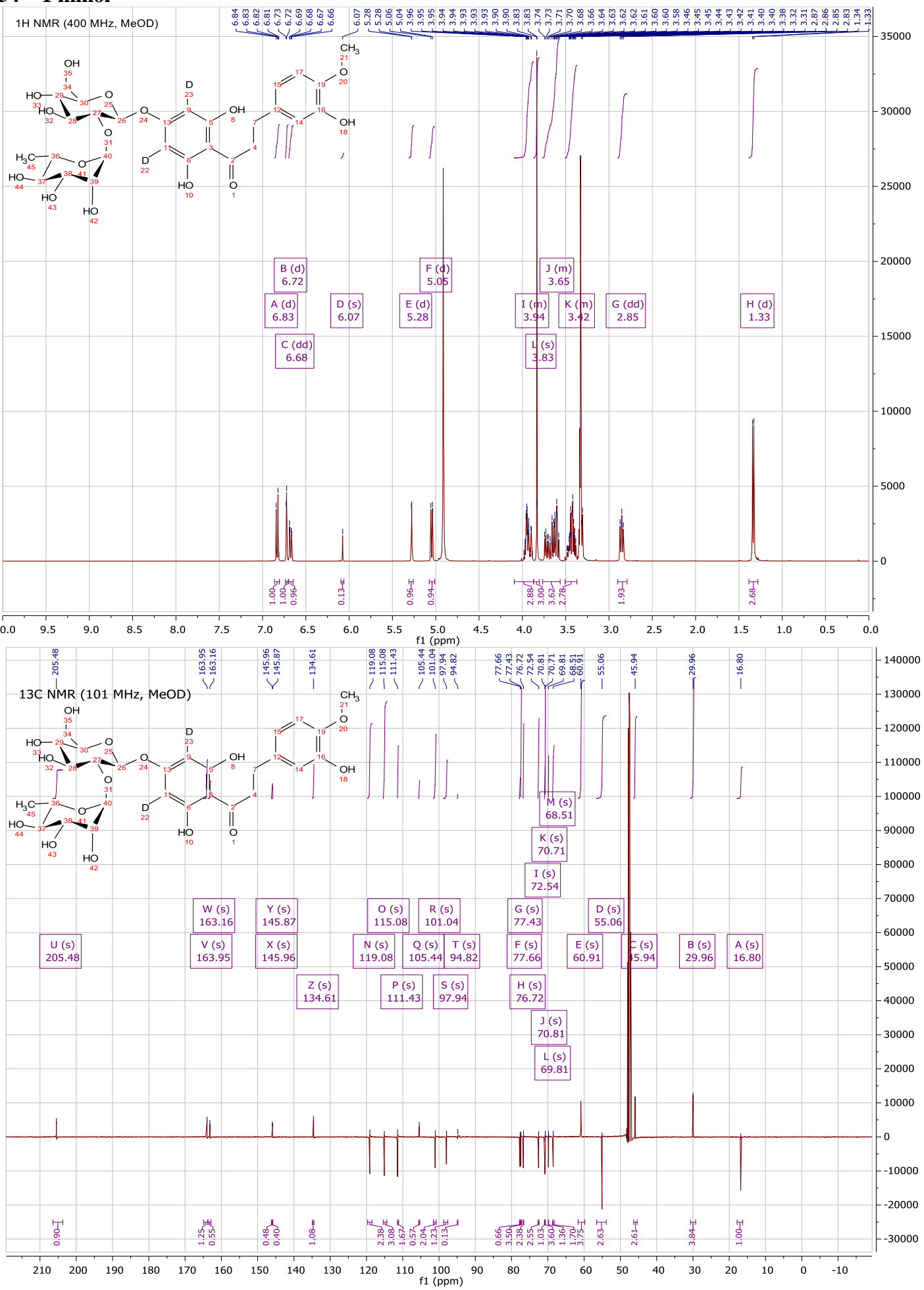



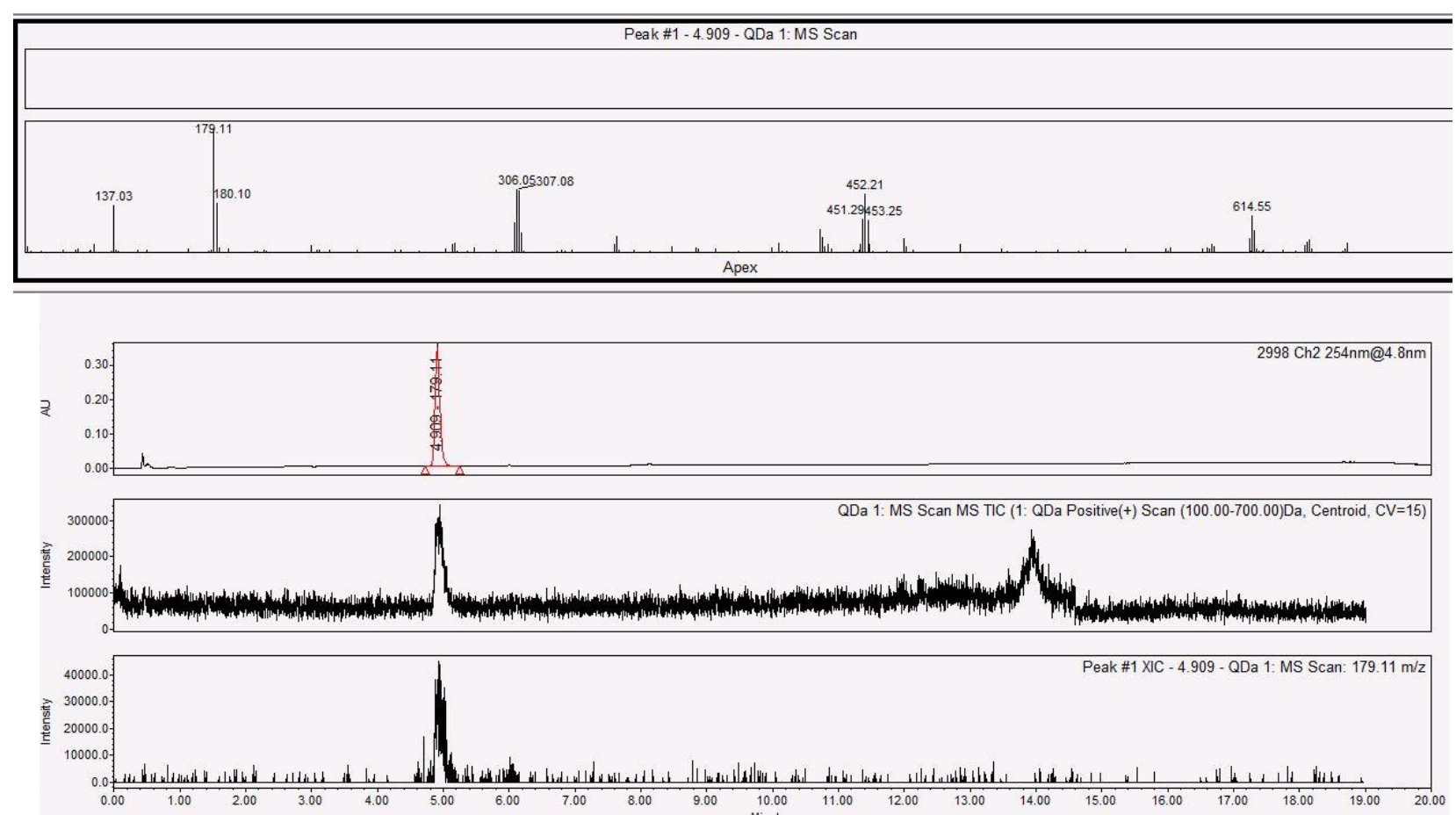


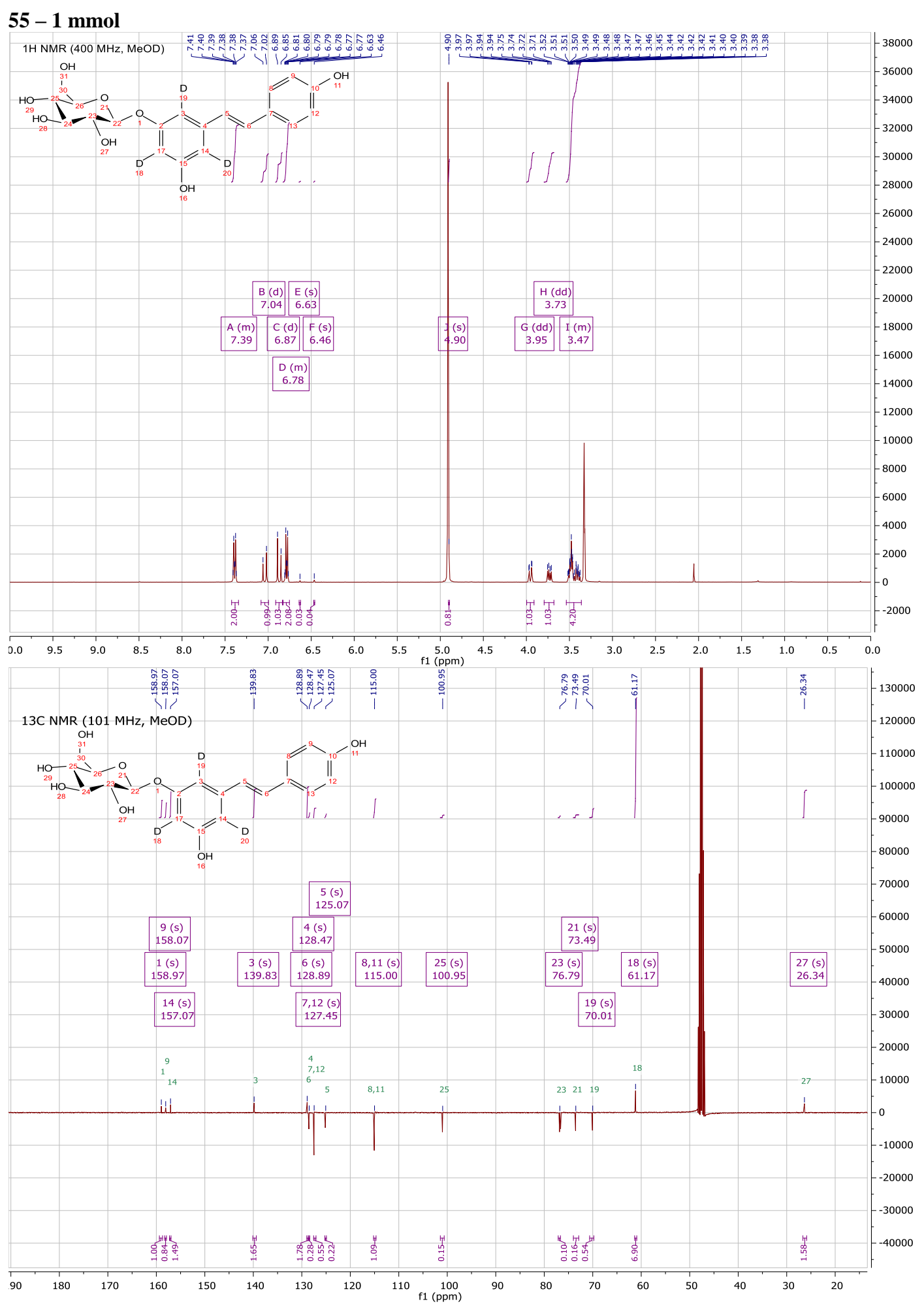



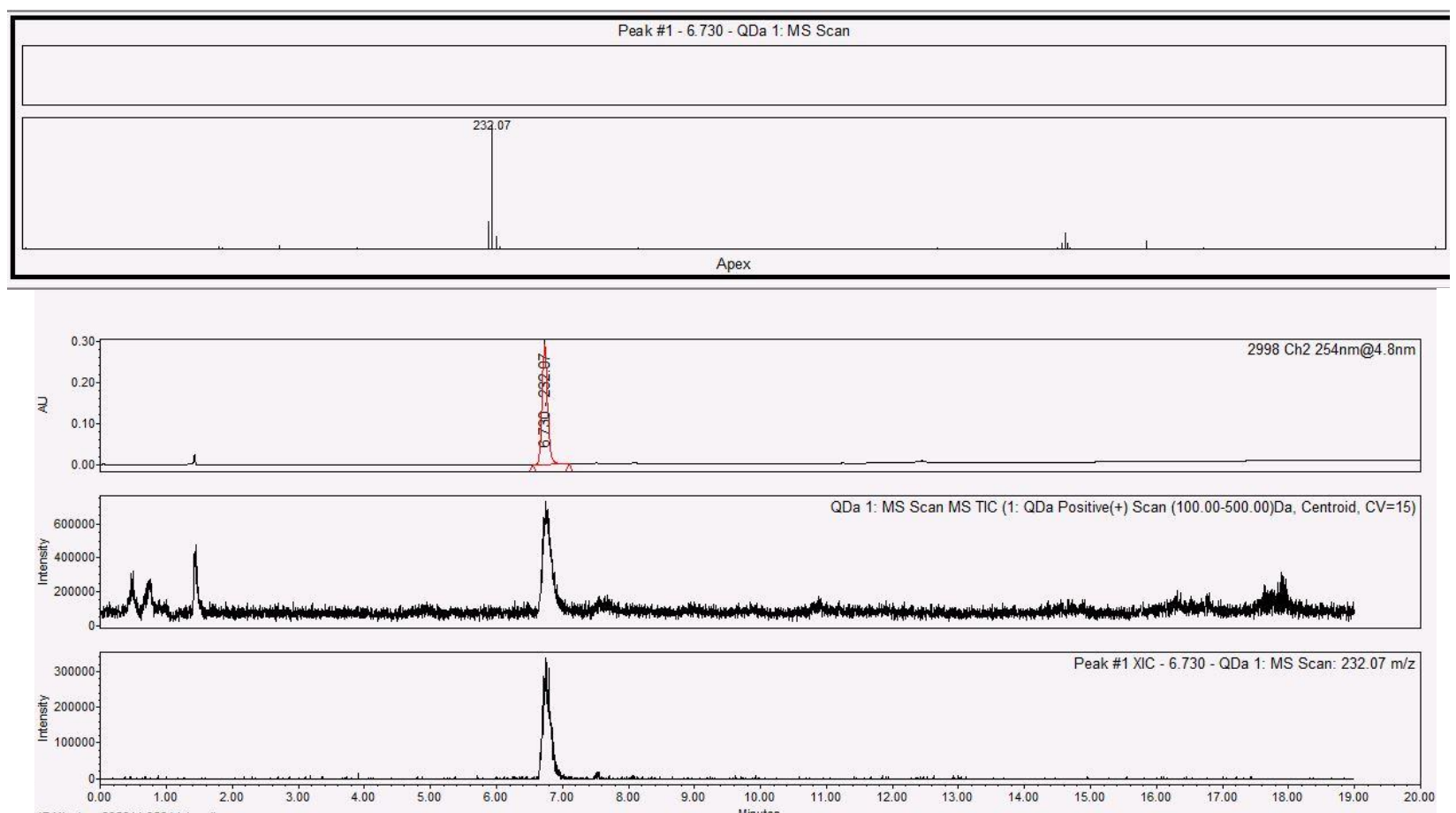


\section{4 - 1 mmol}

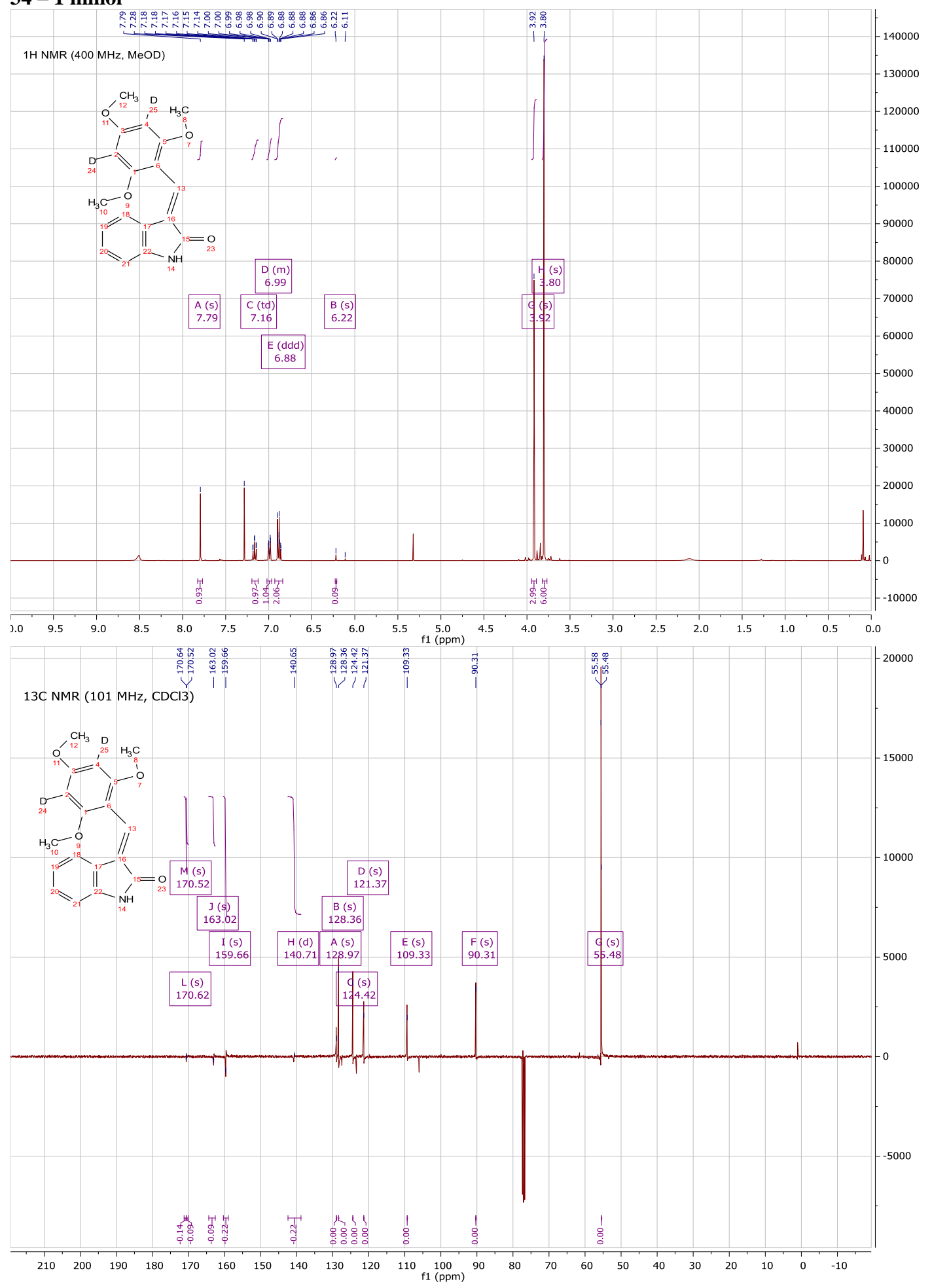



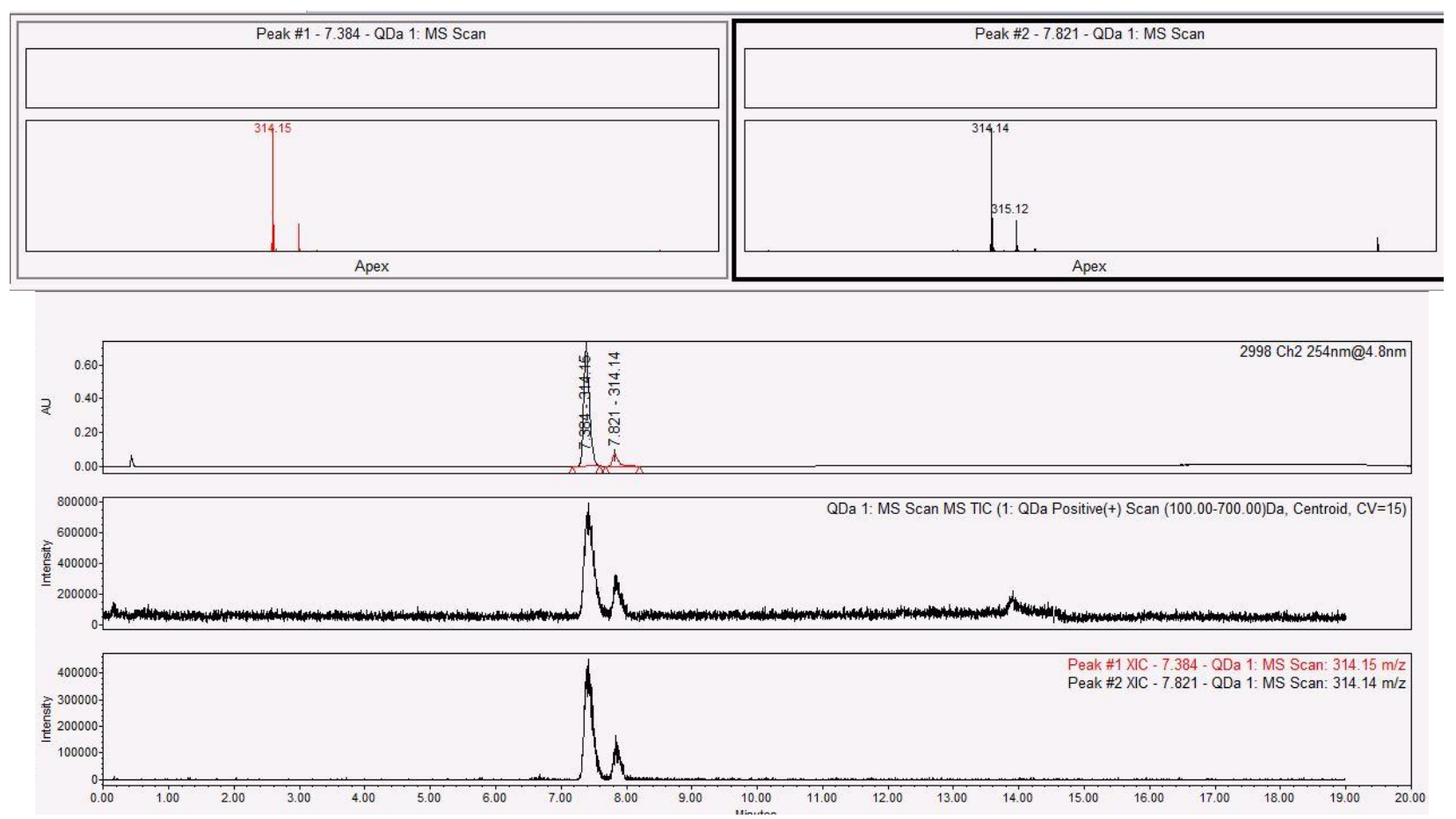


\section{SUPPLEMENTARY MS AND LC/MS INFORMATION}

Continuous/centroid mass spectra of the isotope distribution and LC/MS analysis of the complex substrates

7
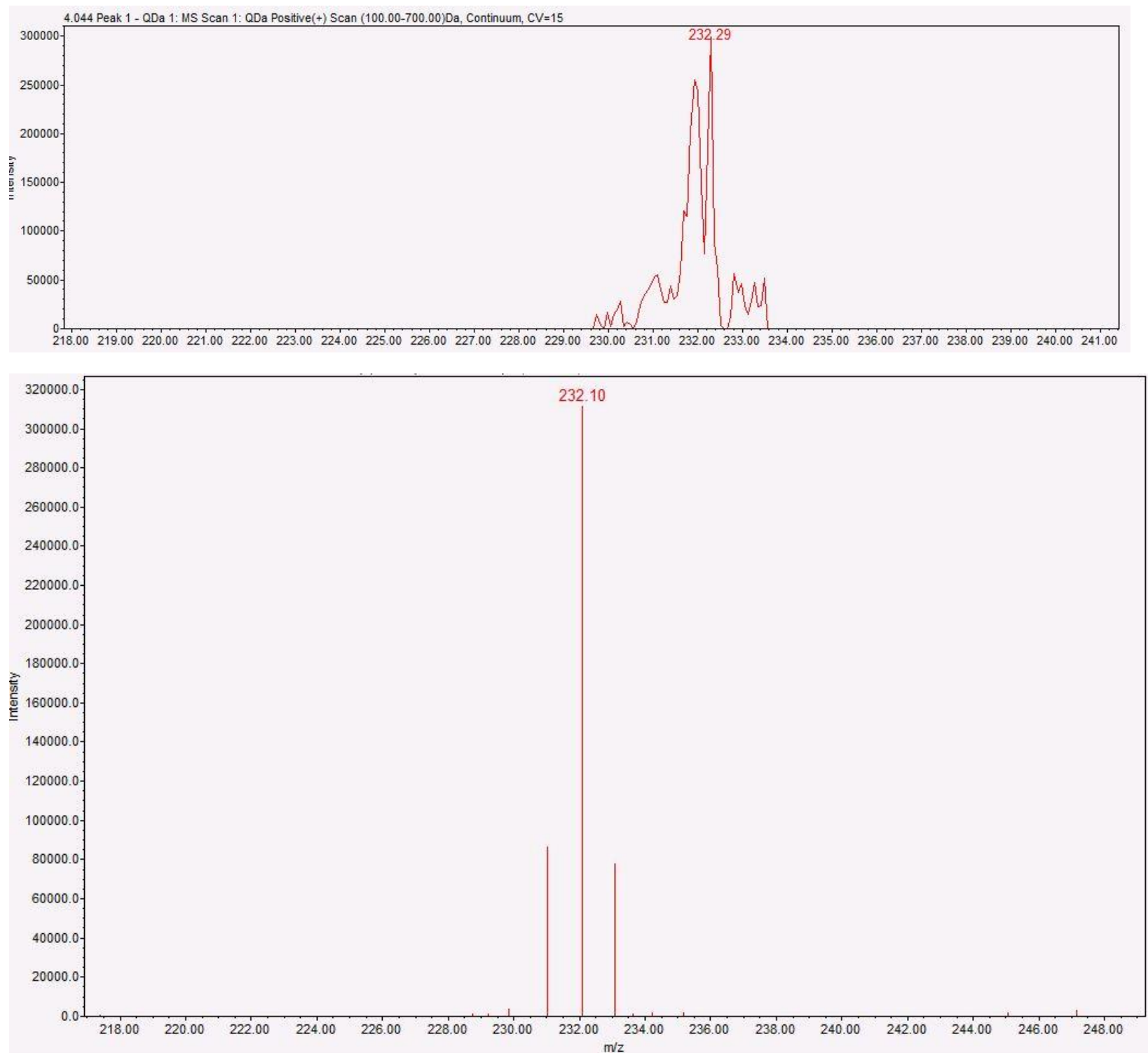


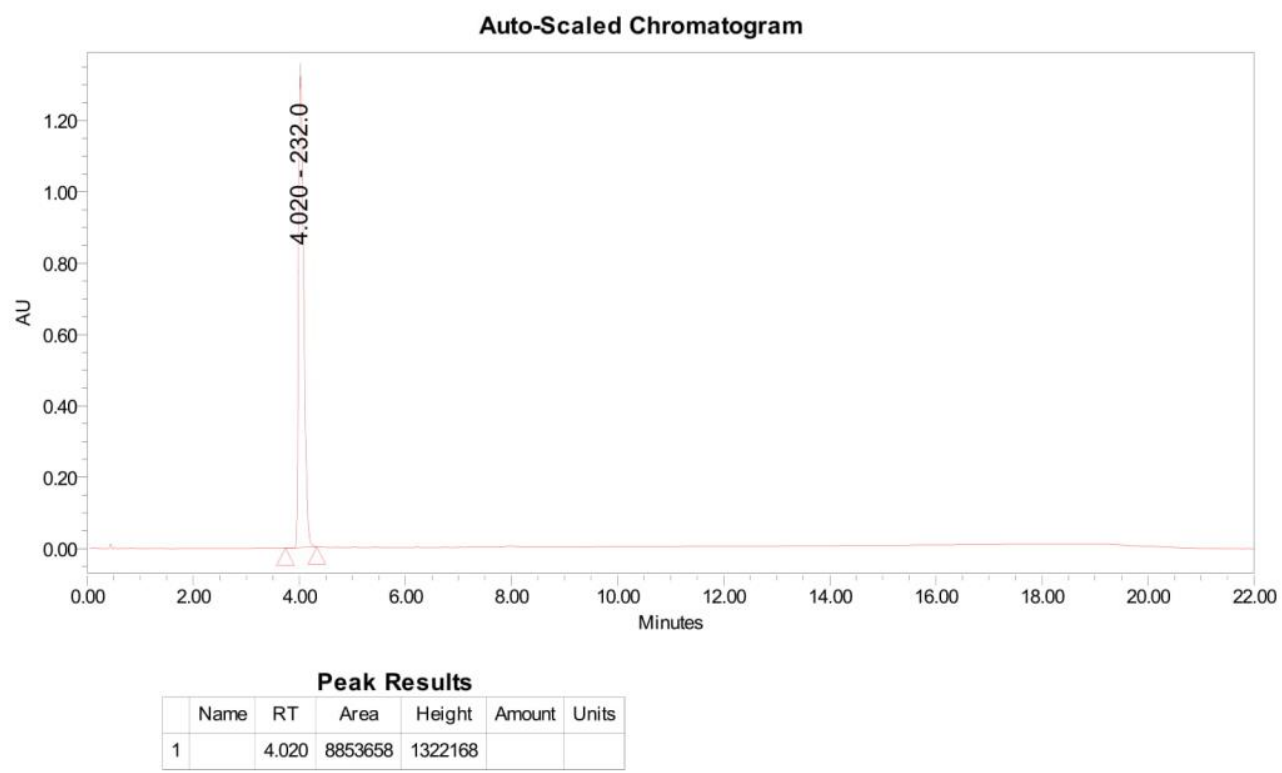


34
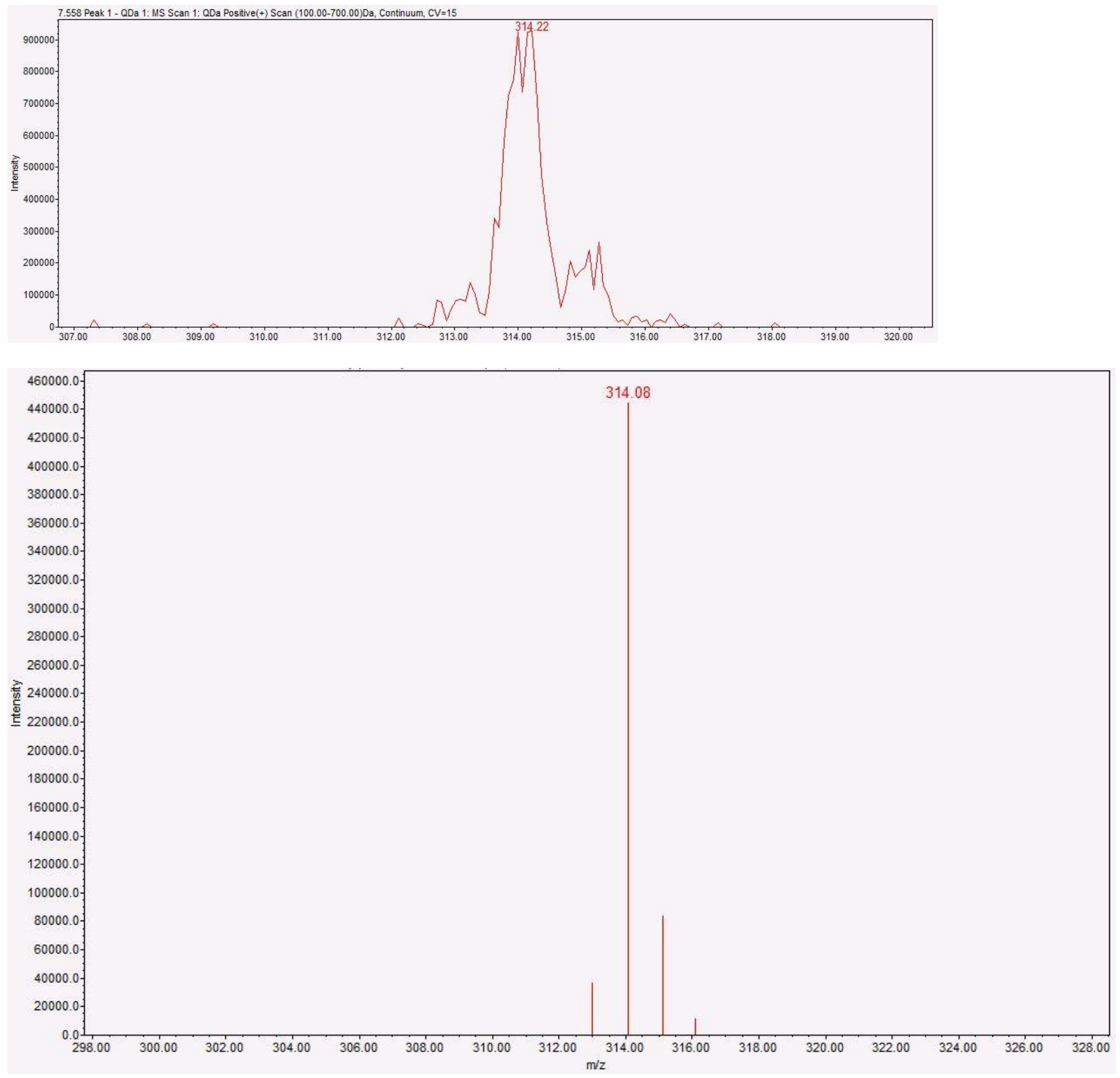


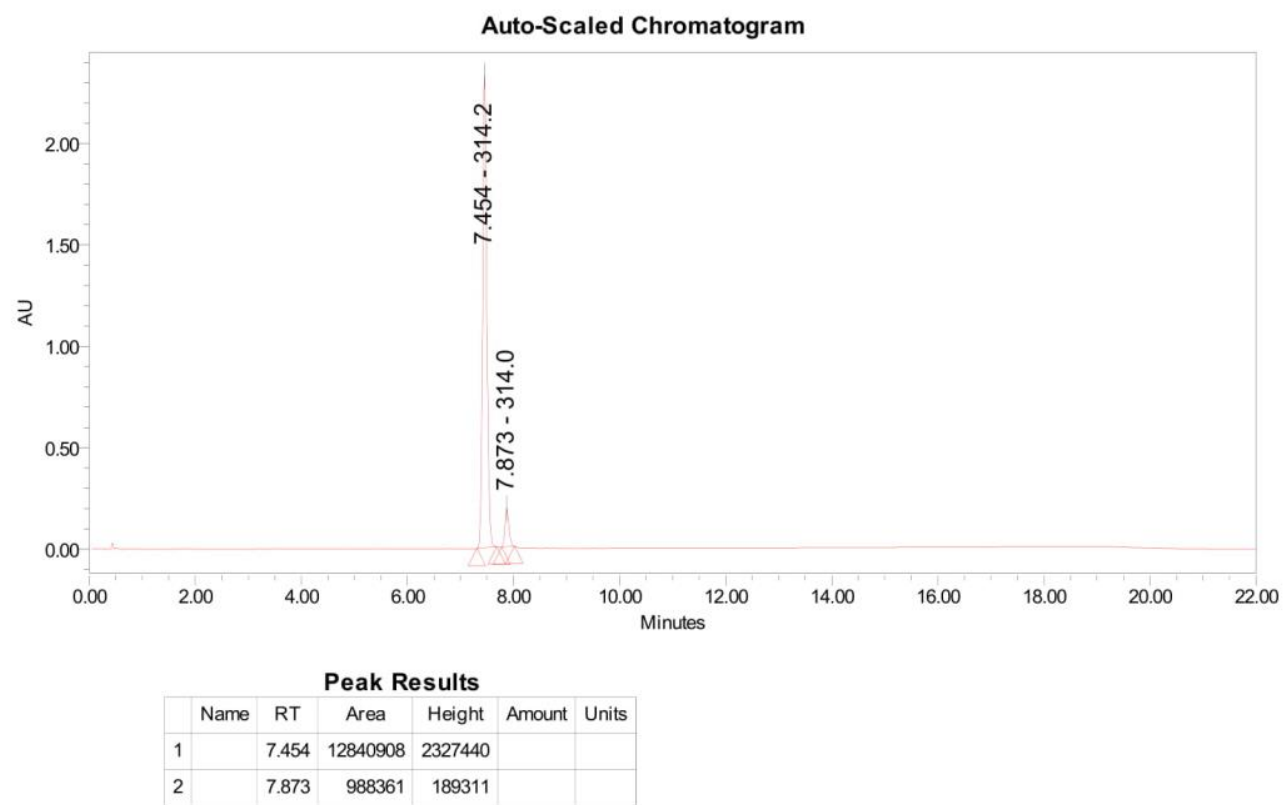



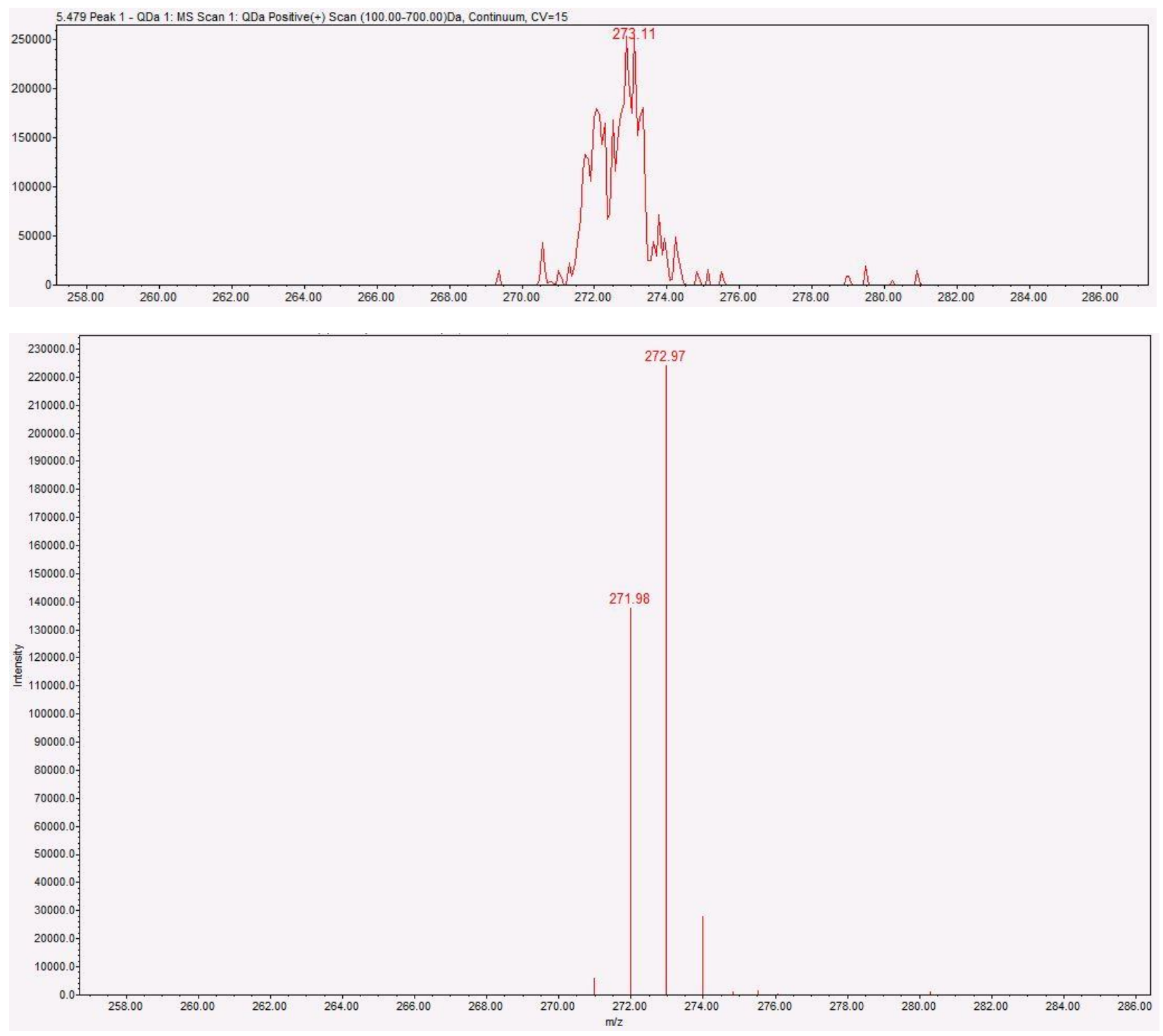


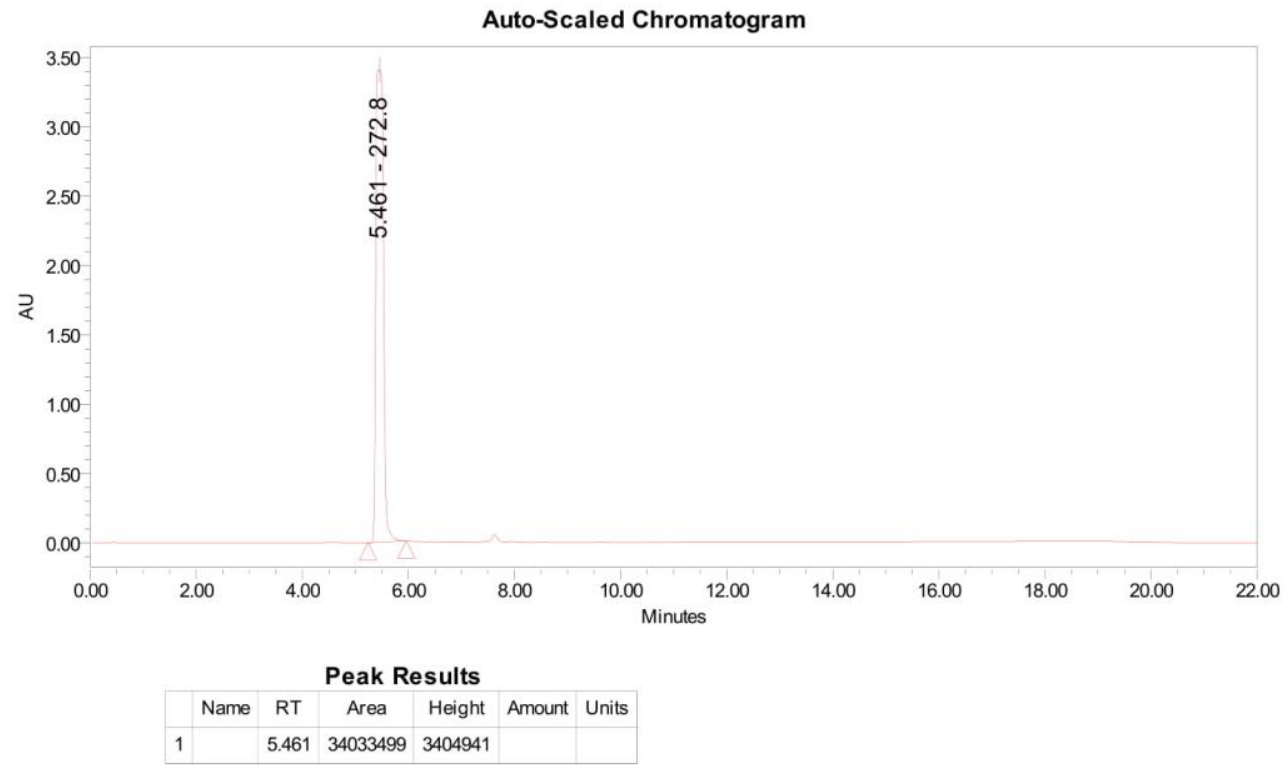



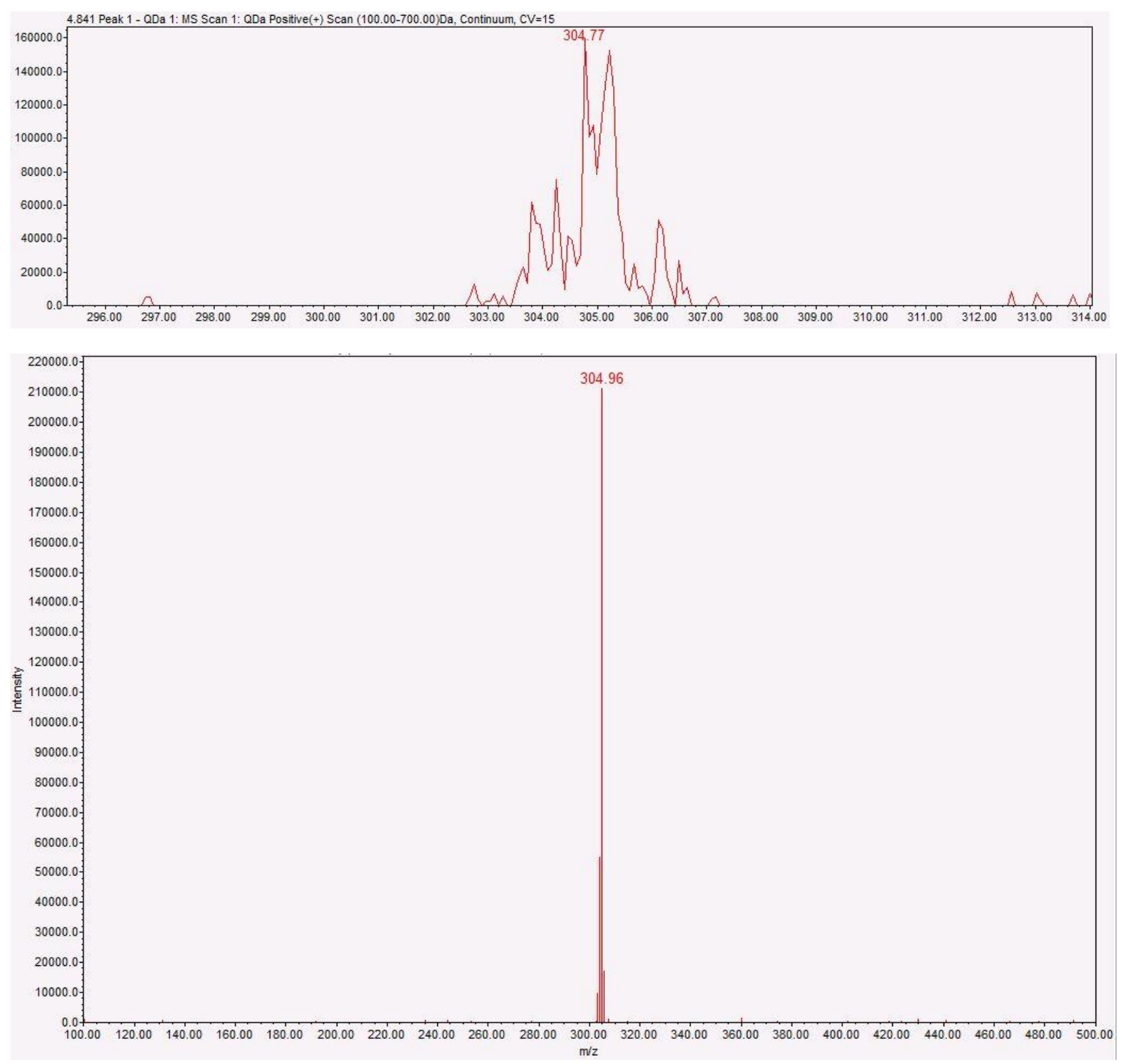


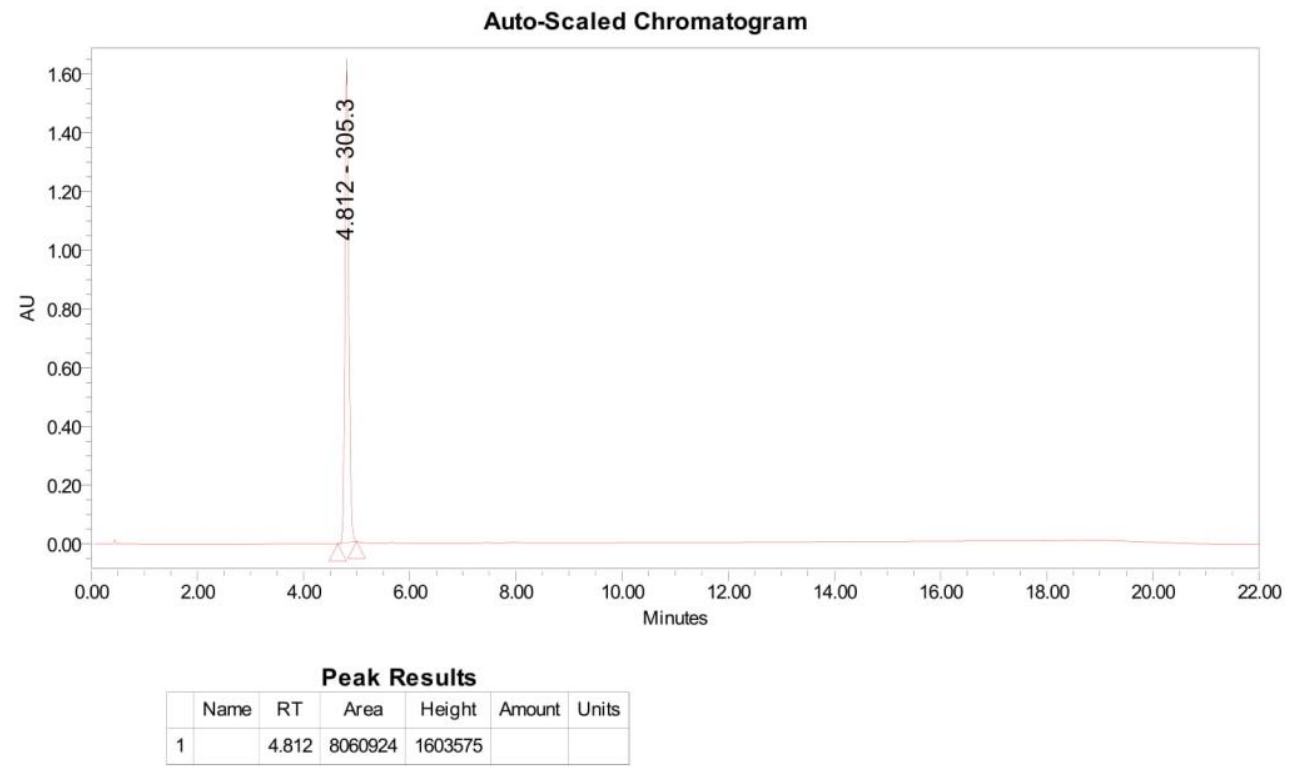

S83 

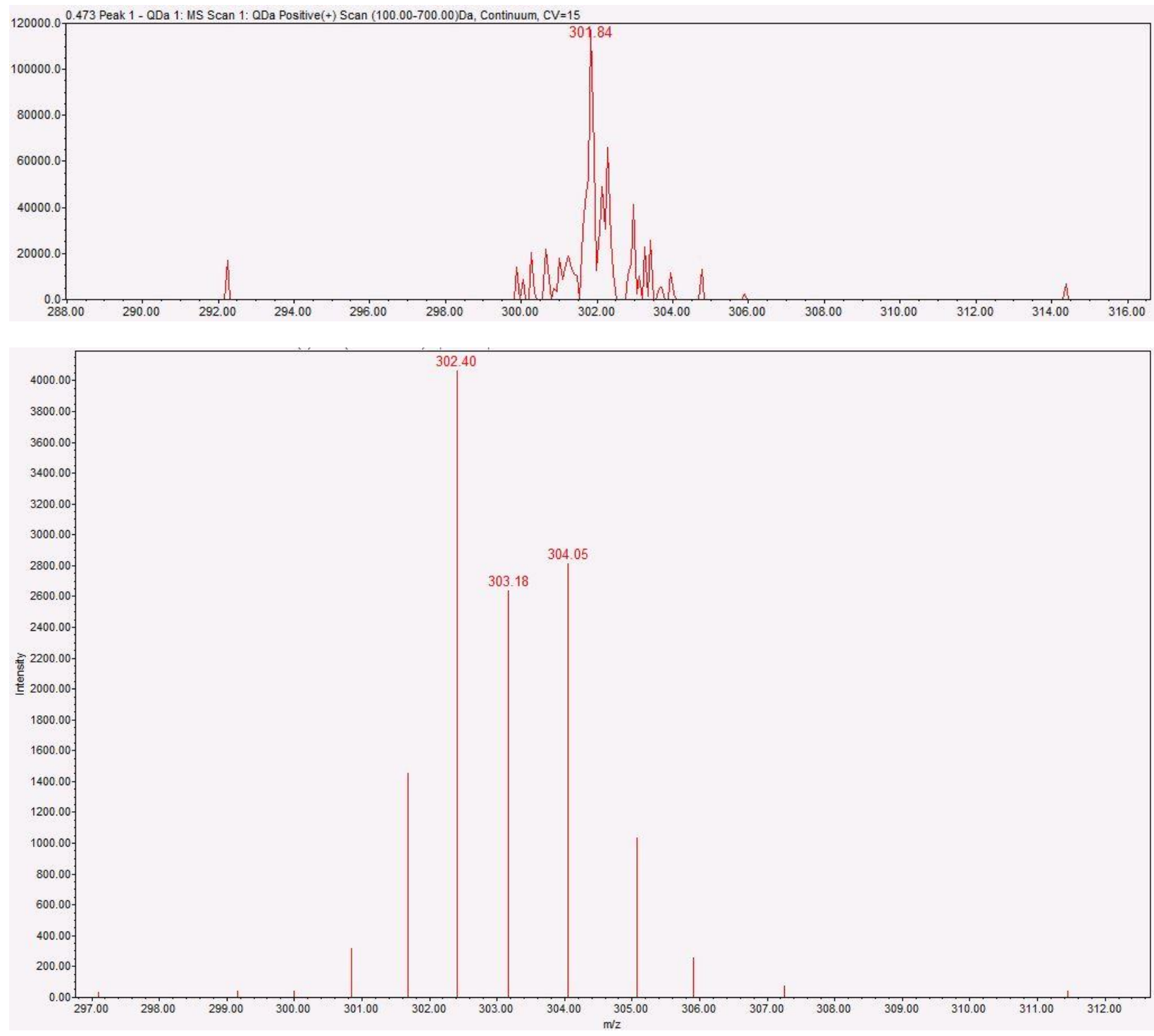

S84 


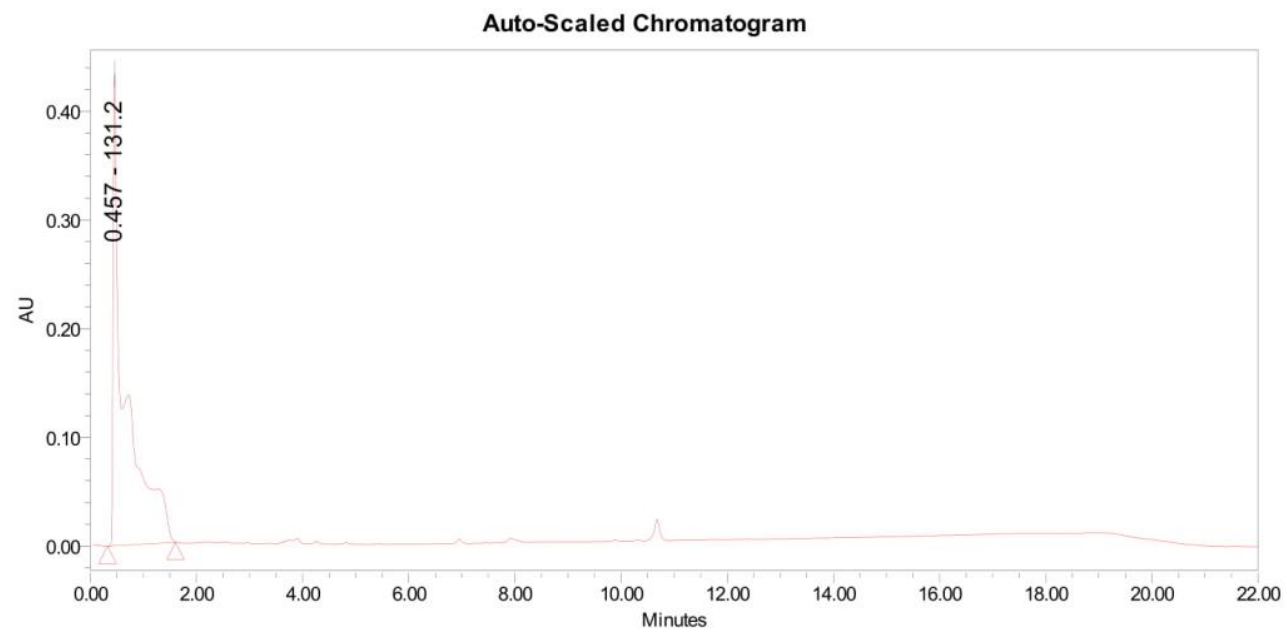

Peak Results

Name RT Area Height Amount Units

\begin{tabular}{|l|l|l|l|}
\hline 1 & 0.457 & 6468409 & 434531 \\
\hline
\end{tabular} 

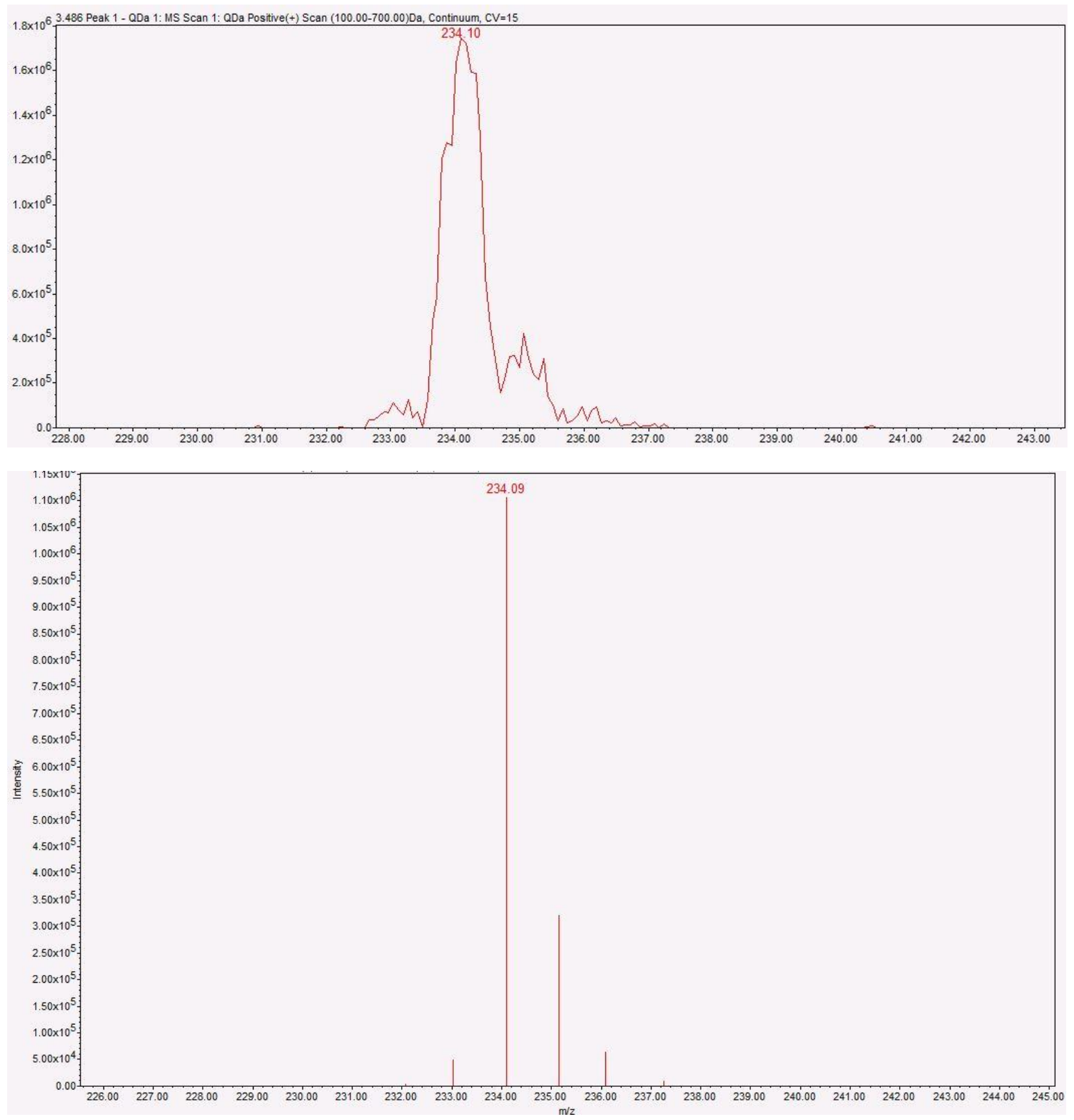


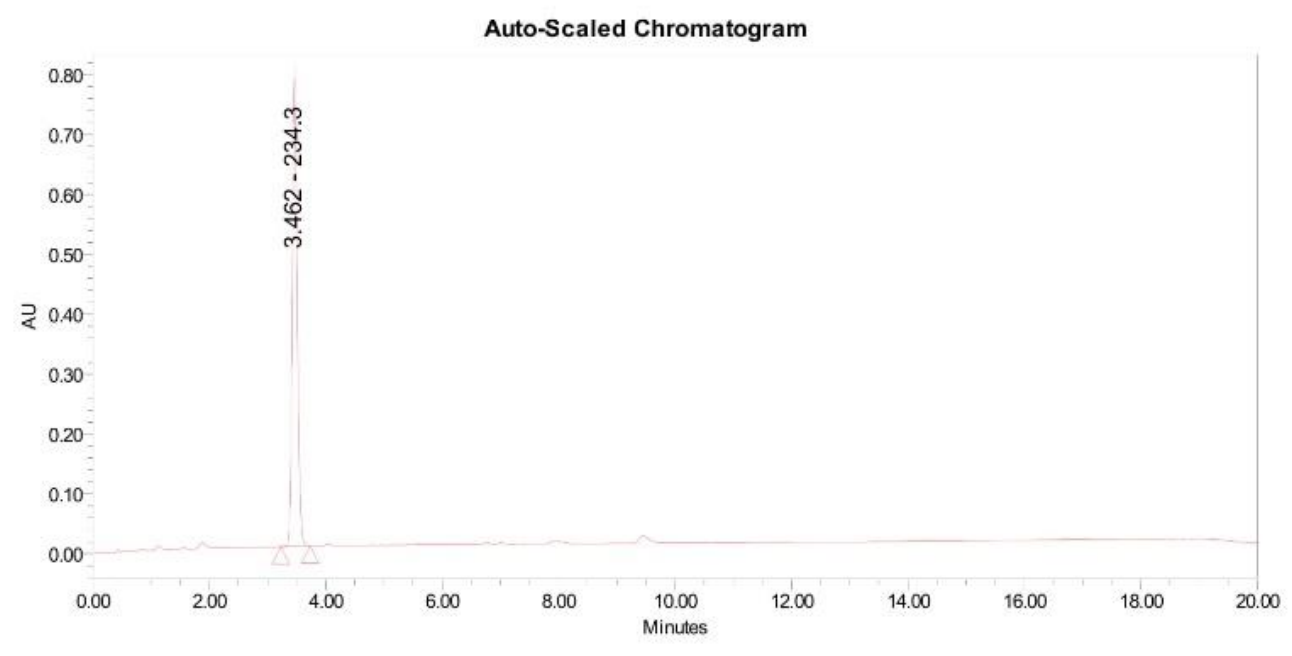

Peak Results

Name RT Area Height Amount Units

\begin{tabular}{l|l|l|l}
1 & 3.462 & 4582306 & 780865
\end{tabular} 

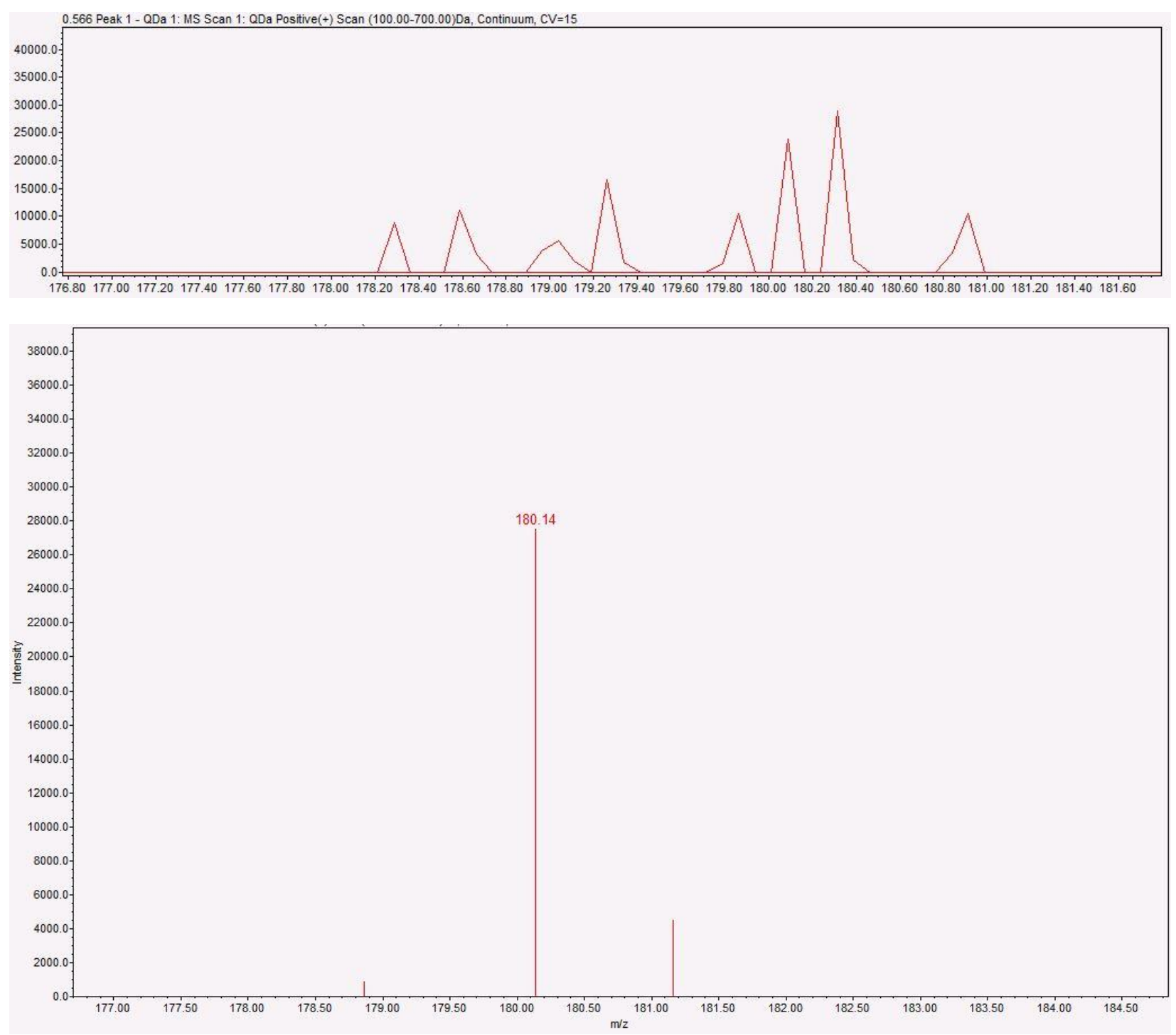


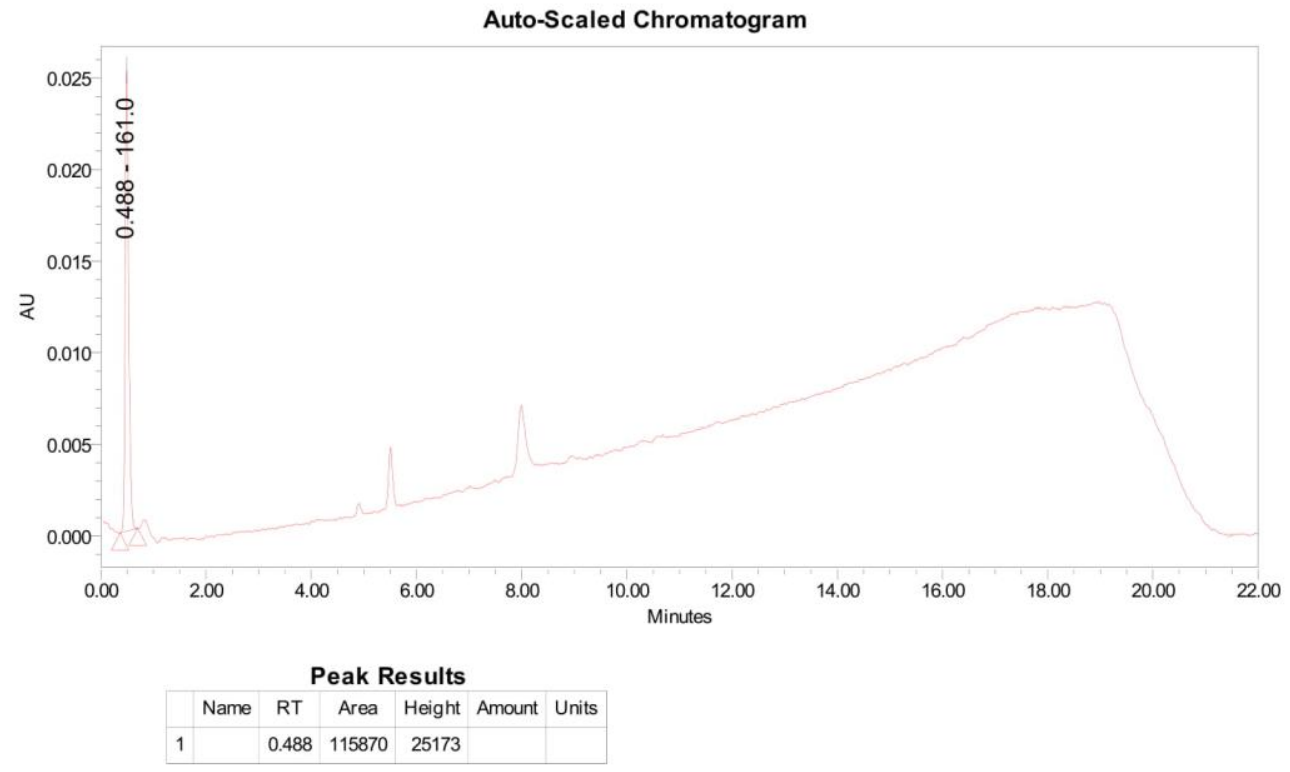



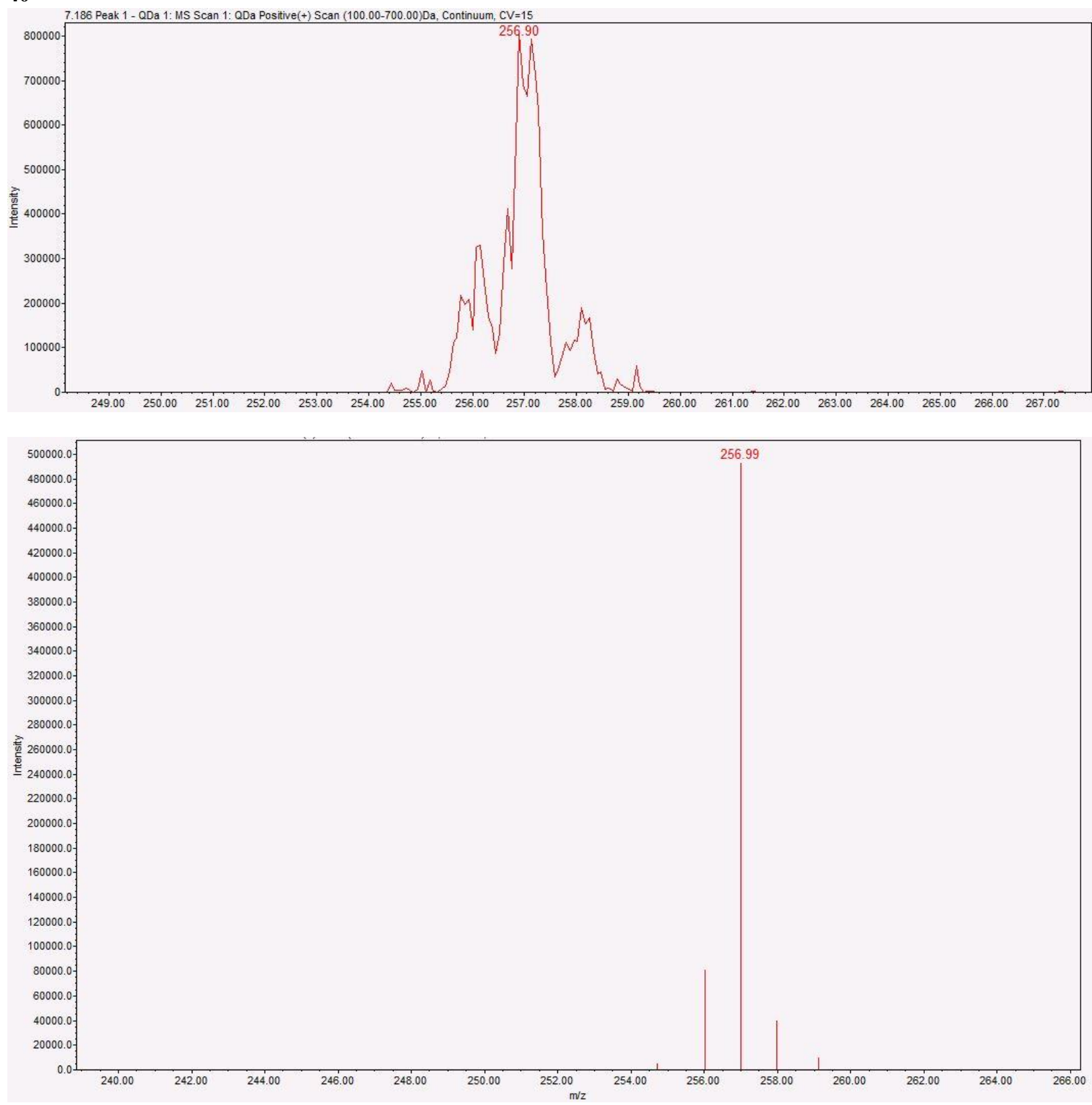


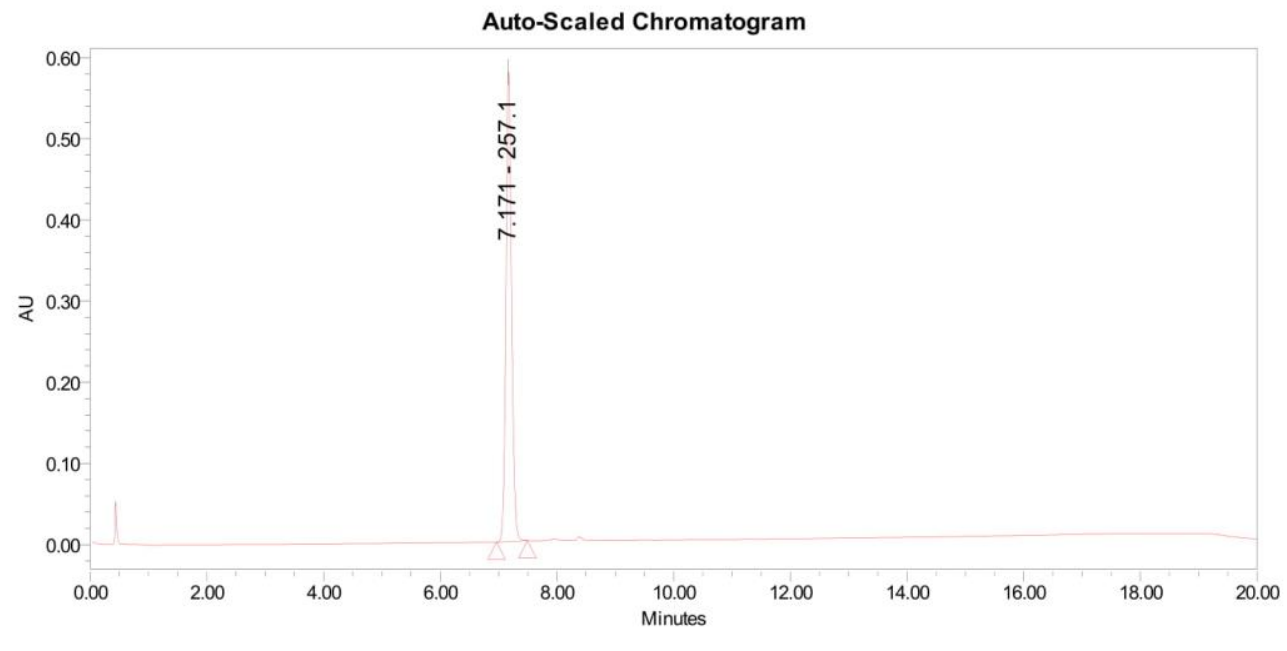

Peak Results

Name RT Area Height Amount Units

\begin{tabular}{|l|l|l|l|}
\hline 1 & 7.171 & 3862916 & 578742 \\
\hline
\end{tabular} 
48
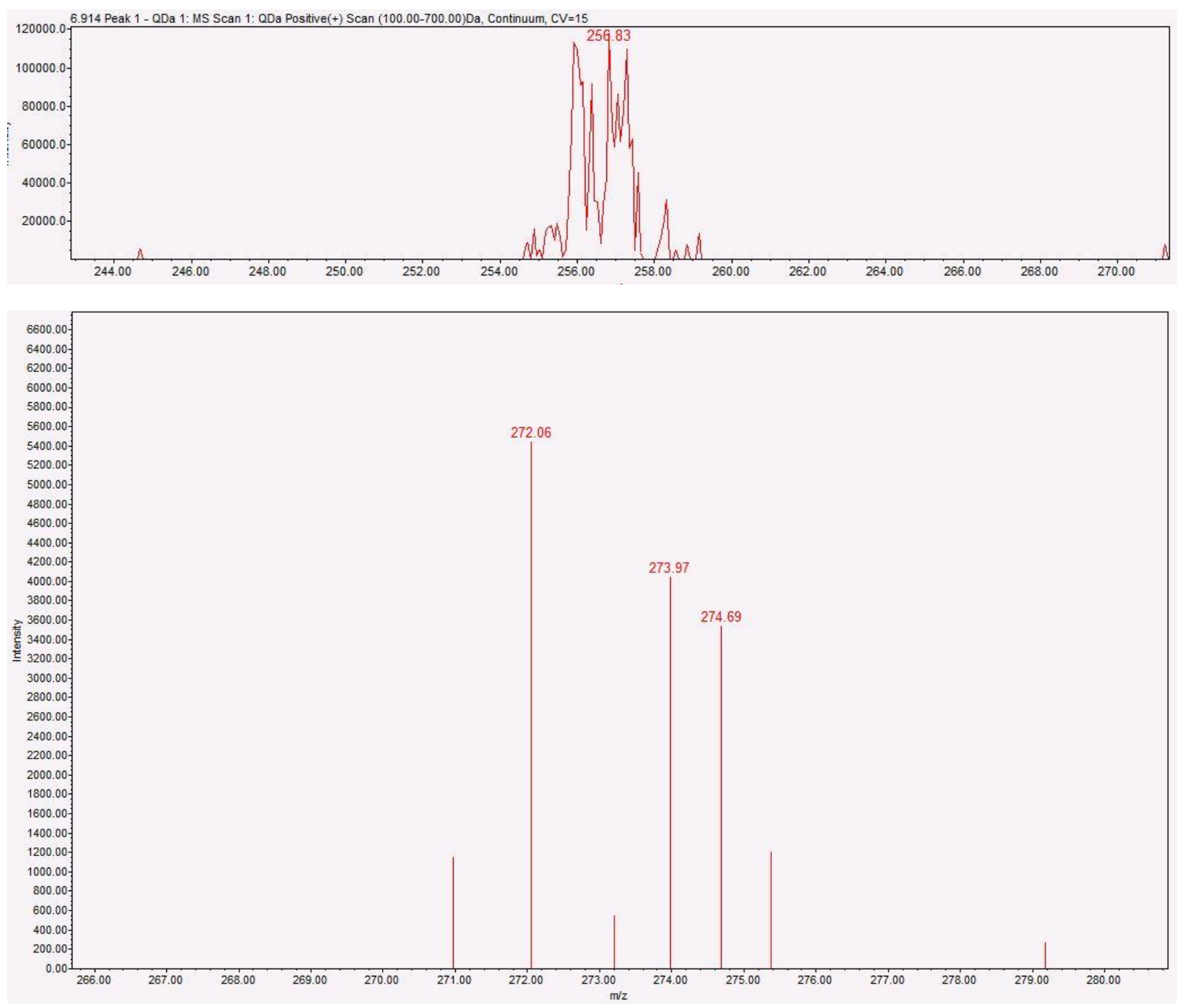

S92 


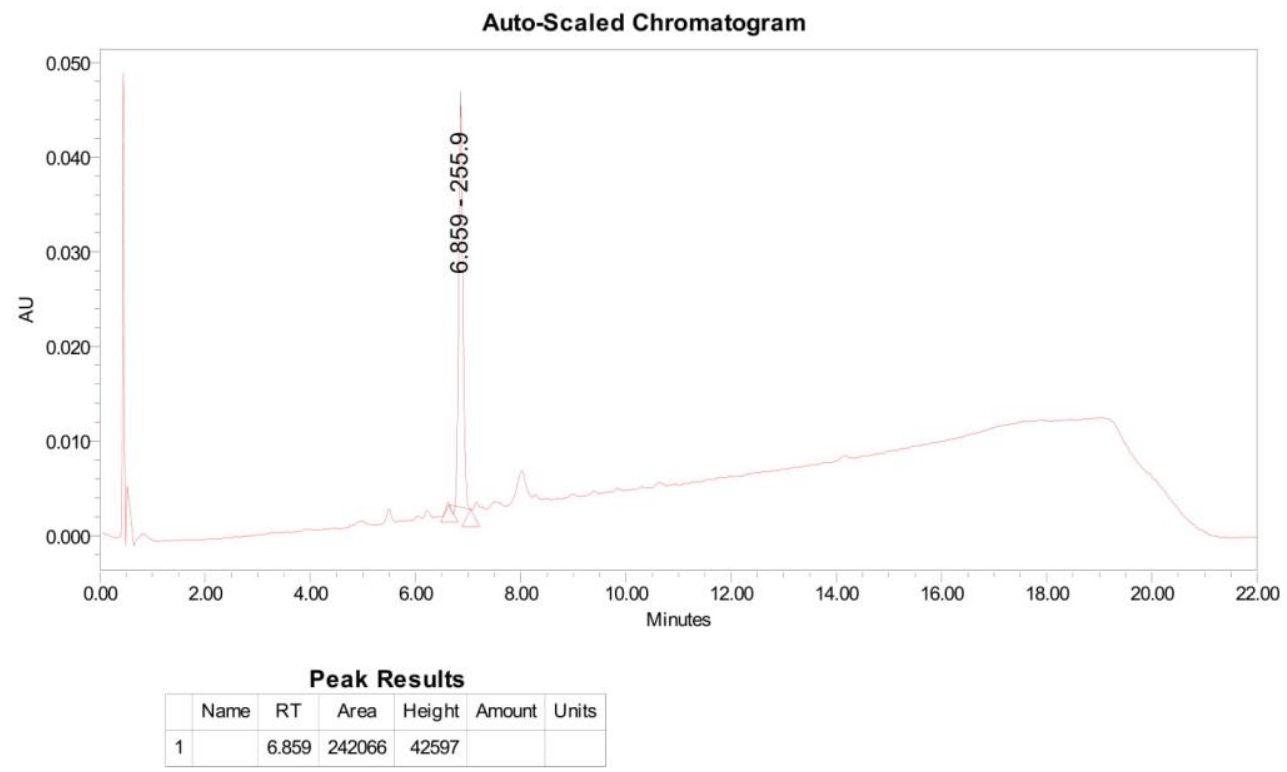



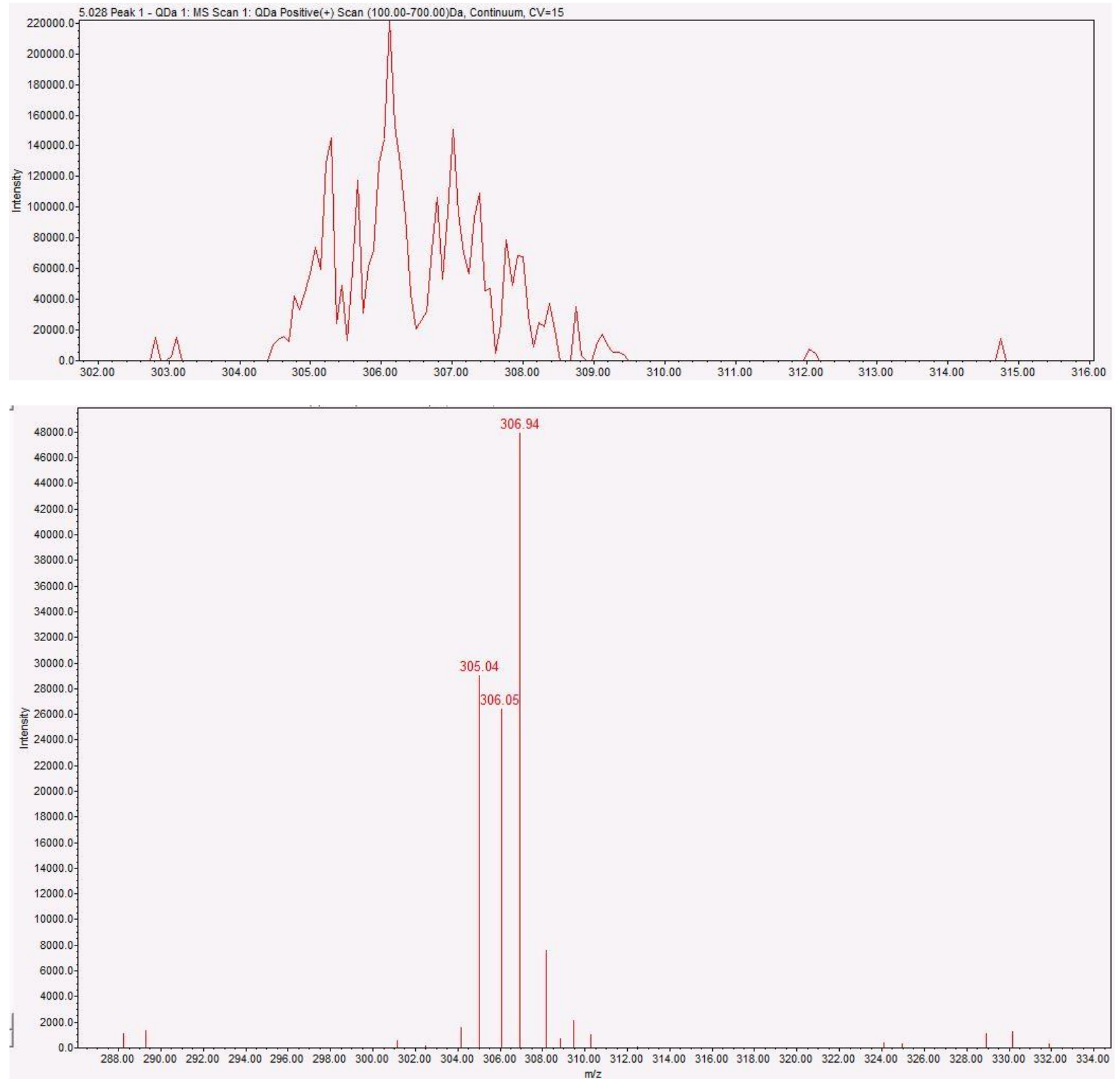


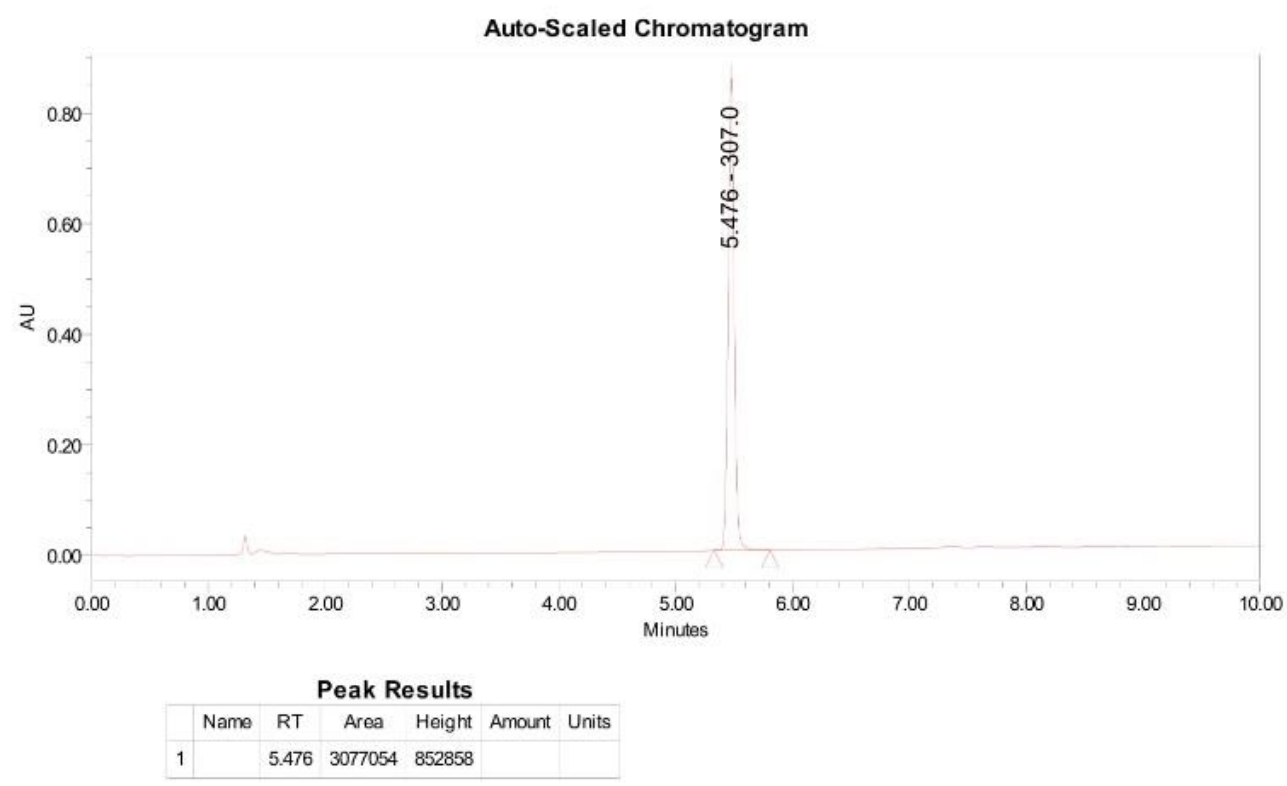



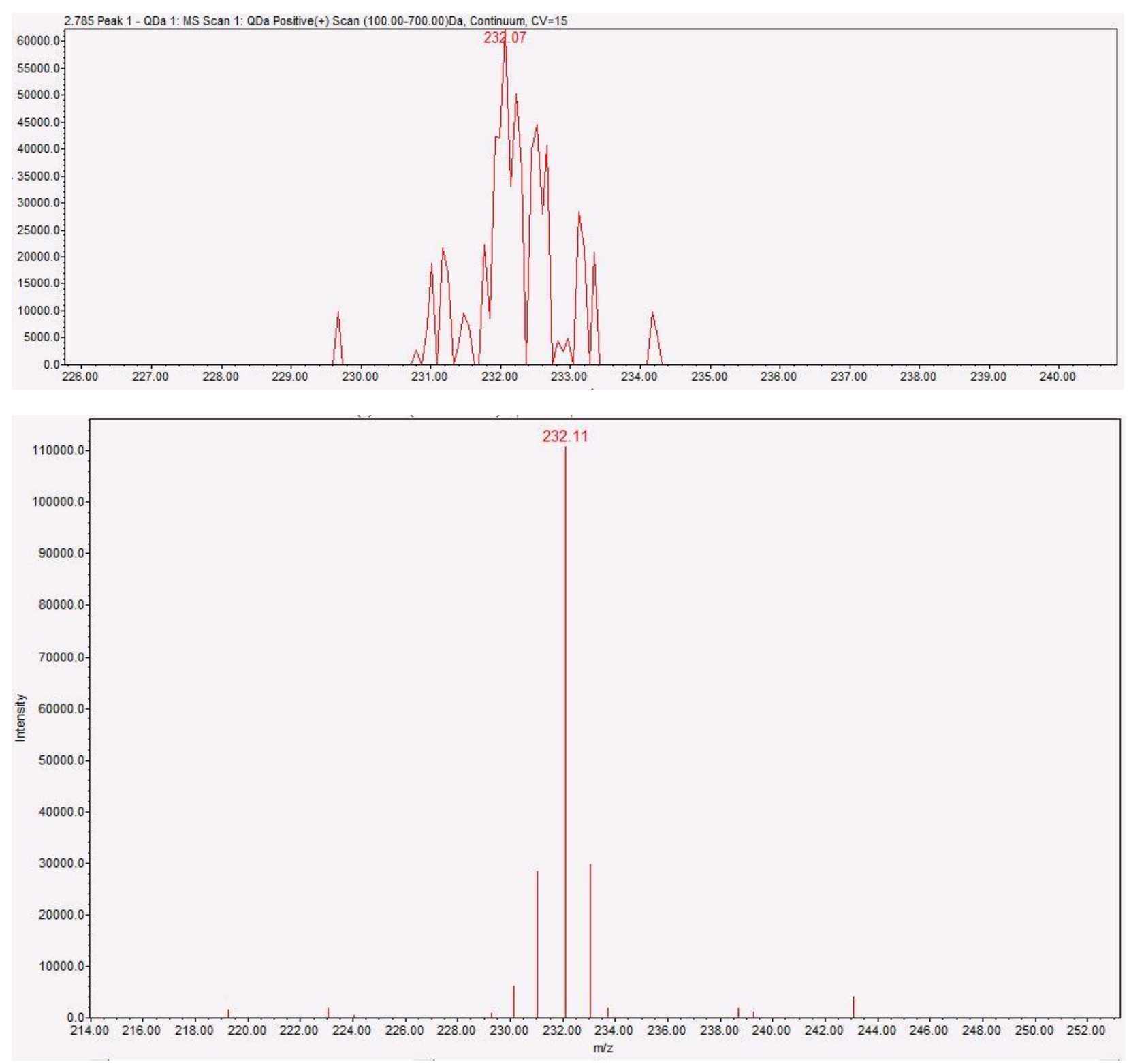


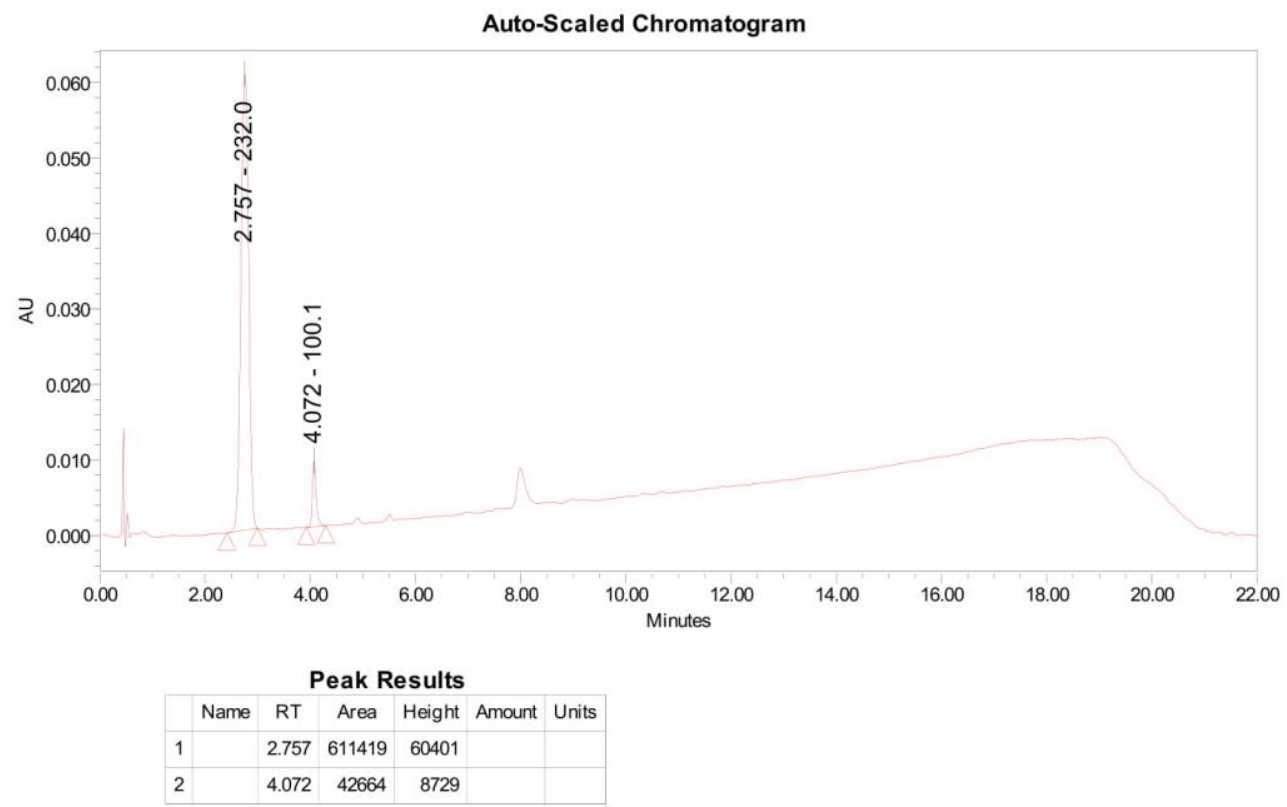



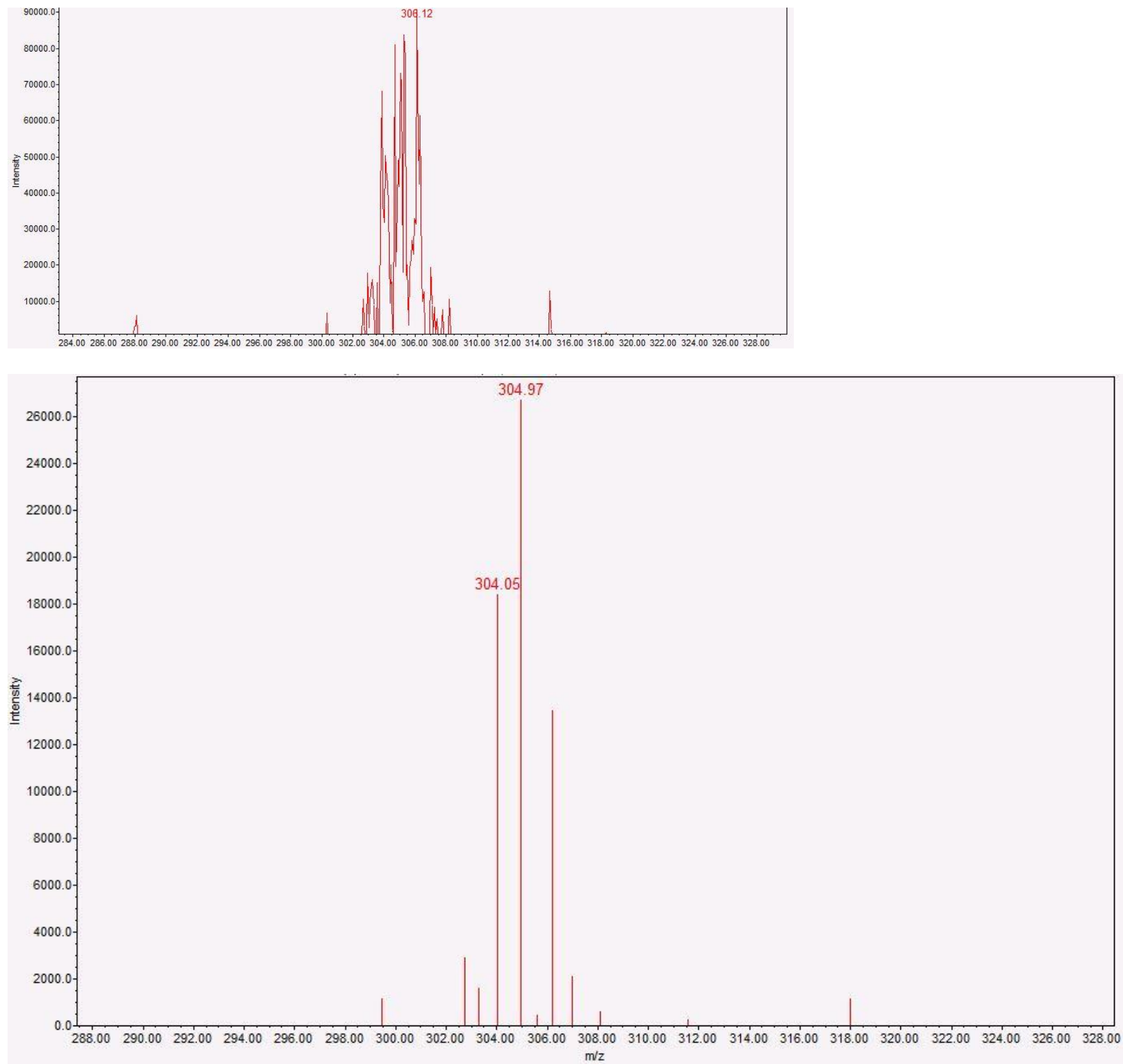


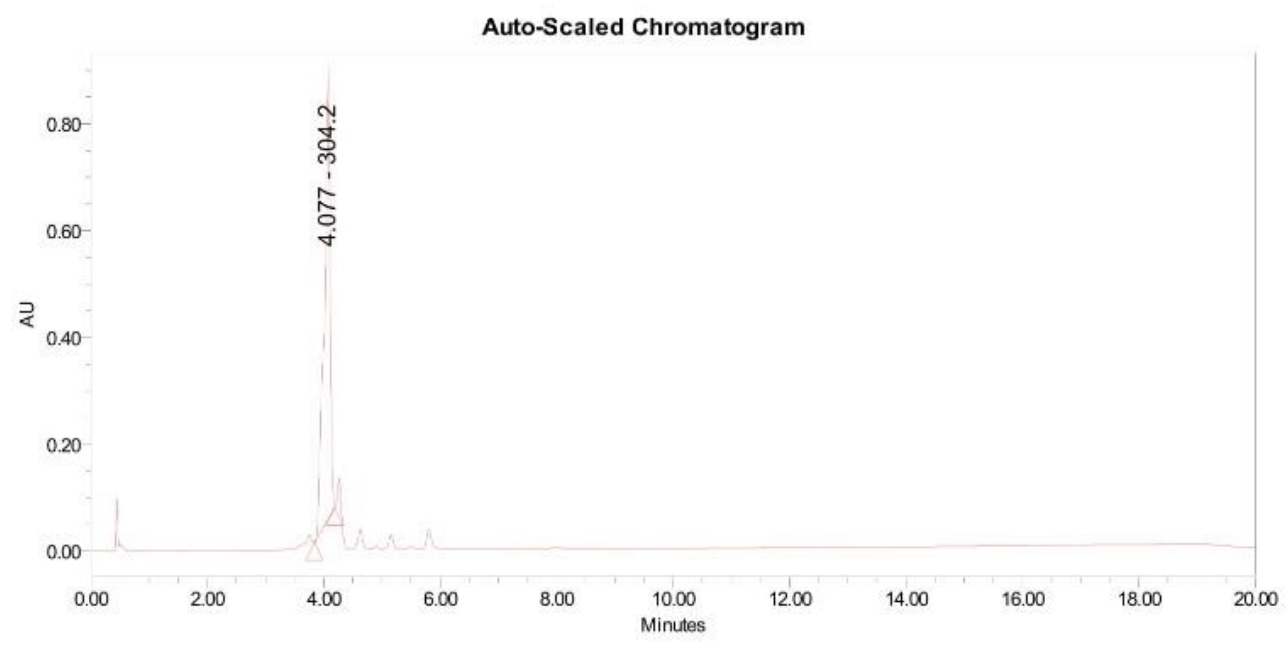

Peak Results

Name RT Area Height Amount Units

\begin{tabular}{ll|l|l|l}
1 & 4.077 & 5684900 & 827077
\end{tabular} 